\title{
LOWER SEMICONTINUITY AND RELAXATION OF LINEAR-GROWTH INTEGRAL FUNCTIONALS UNDER PDE CONSTRAINTS
}

\author{
ADOLFO ARROYO-RABASA, GUIDO DE PHILIPPIS, AND FILIP RINDLER
}

\begin{abstract}
We show general lower semicontinuity and relaxation theorems for linear-growth integral functionals defined on vector measures that satisfy linear PDE side constraints (of arbitrary order). These results generalize several known lower semicontinuity and relaxation theorems for $\mathrm{BV}, \mathrm{BD}$, and for more general first-order linear PDE side constrains. Our proofs are based on recent progress in the understanding of singularities of measure solutions to linear PDEs and of the generalized convexity notions corresponding to these PDE constraints.
\end{abstract}

KEYWORDS: Lower semicontinuity, functional on measures, $\mathscr{A}$-quasiconvexity, generalized Young measure.

DATE: December 27, 2017.

\section{INTRODUCTION}

The theory of linear-growth integral functionals defined on vector-valued measures satisfying PDE constraints is central to many questions of the calculus of variations. In particular, their relaxation and lower semicontinuity properties have attracted a lot of attention, see for instance [AD92, FM93, FM99, FLM04, KR10b, Rin11, BCMS13]. In the present work we unify and extend a large number of these results by proving general lower semicontinuity and relaxation theorems for such functionals. Our proofs are based on recent advances in the understanding of the singularities that may occur in measures satisfying (under-determined) linear PDEs.

Concretely, let $\Omega \subset \mathbb{R}^{d}$ be an open and bounded subset with $\mathscr{L}^{d}(\partial \Omega)=0$ and consider the functional

$$
\mathscr{F}^{\#}[\mu]:=\int_{\Omega} f\left(x, \frac{\mathrm{d} \mu}{\mathrm{d} \mathscr{L}^{d}}(x)\right) \mathrm{d} x+\int_{\Omega} f^{\#}\left(x, \frac{\mathrm{d} \mu^{s}}{\mathrm{~d}\left|\mu^{s}\right|}(x)\right) \mathrm{d}\left|\mu^{s}\right|(x),
$$

defined for finite vector Radon measures $\mu \in \mathscr{M}\left(\Omega ; \mathbb{R}^{N}\right)$ on $\Omega$ with values in $\mathbb{R}^{N}$. Here, $f: \Omega \times \mathbb{R}^{N} \rightarrow \mathbb{R}$ is a Borel integrand that has linear growth at infinity, i.e.,

$$
|f(x, A)| \leq M(1+|A|) \quad \text { for all }(x, A) \in \Omega \times \mathbb{R}^{N},
$$

whereby the (generalized) recession function

$$
f^{\#}(x, A):=\limsup _{\substack{x^{\prime} \rightarrow x \\ A^{\prime} \rightarrow A \\ t \rightarrow \infty}} \frac{f\left(x^{\prime}, t A^{\prime}\right)}{t}, \quad(x, A) \in \bar{\Omega} \times \mathbb{R}^{N},
$$

takes only finite values. Furthermore, on the candidate measures $\mu \in \mathscr{M}\left(\Omega ; \mathbb{R}^{N}\right)$ we impose the $k$ th-order linear PDE side constraint

$$
\mathscr{A} \mu:=\sum_{|\alpha| \leq k} A_{\alpha} \partial^{\alpha} \mu=0 \quad \text { in the sense of distributions. }
$$

The coefficient matrices $A_{\alpha} \in \mathbb{R}^{n} \otimes \mathbb{R}^{N}$ are assumed to be constant and we write $\partial^{\alpha}=\partial_{1}^{\alpha_{1}} \ldots \partial_{d}^{\alpha_{d}}$ for every multi-index $\alpha=\left(\alpha_{1}, \ldots, \alpha_{d}\right) \in(\mathbb{N} \cup\{0\})^{d}$ with $|\alpha|:=$ 
$\left|\alpha_{1}\right|+\cdots+\left|\alpha_{d}\right| \leq k$. We call measures $\mu \in \mathscr{M}\left(\Omega ; \mathbb{R}^{N}\right)$ with $\mathscr{A} \mu=0$ in the sense of distributions $\mathscr{A}$-free.

We will also assume that $\mathscr{A}$ satisfies Murat's constant rank condition (see [Mur81, FM99]), that is, there exists $r \in \mathbb{N}$ such that

$$
\operatorname{rank} \mathbb{A}^{k}(\xi)=r \quad \text { for all } \xi \in \mathbb{S}^{d-1},
$$

where

$$
\mathbb{A}^{k}(\xi):=(2 \pi \mathrm{i})^{k} \sum_{|\alpha|=k} \xi^{\alpha} A_{\alpha}, \quad \xi^{\alpha}=\xi_{1}^{\alpha_{1}} \ldots \xi_{d}^{\alpha_{d}},
$$

is the principal symbol of $\mathscr{A}$. We also recall the notion of wave cone associated to $\mathscr{A}$, which plays a fundamental role in the study of $\mathscr{A}$-free fields and first originated in the theory of compensated compactness [Tar79, Tar83, Mur78, Mur79, Mur81, DiP85].

Definition 1.1. Let $\mathscr{A}$ be a kth-order linear PDE operator as above. The wave cone associated to $\mathscr{A}$ is the set

$$
\Lambda_{\mathscr{A}}:=\bigcup_{|\xi|=1} \operatorname{ker} \mathbb{A}^{k}(\xi) \subset \mathbb{R}^{N} .
$$

Note that the wave cone contains those amplitudes along which it is possible to construct highly oscillating $\mathscr{A}$-free fields. More precisely, if $\mathscr{A}$ is homogeneous, i.e., $\mathscr{A}=\sum_{|\alpha|=k} A_{\alpha} \partial^{\alpha}$, then $P_{0} \in \Lambda_{\mathscr{A}}$ if and only if there exists $\xi \neq 0$ such that

$$
\mathscr{A}\left(P_{0} h(x \cdot \xi)\right)=0 \quad \text { for all } h \in \mathrm{C}^{k}(\mathbb{R}) .
$$

Our first main theorem concerns the case when $f$ is $\mathscr{A}^{k}$-quasiconvex in its second argument, where

$$
\mathscr{A}^{k}:=\sum_{|\alpha|=k} A_{\alpha} \partial^{\alpha}
$$

is the principal part of $\mathscr{A}$. Recall from [FM99] that a Borel function $h: \mathbb{R}^{N} \rightarrow \mathbb{R}$ is called $\mathscr{A}^{k}$-quasiconvex if

$$
h(A) \leq \int_{Q} h(A+w(y)) \mathrm{d} y
$$

for all $A \in \mathbb{R}^{N}$ and all $Q$-periodic $w \in \mathrm{C}^{\infty}\left(Q ; \mathbb{R}^{N}\right)$ such that $\mathscr{A}^{k} w=0$ and $\int_{Q} w \mathrm{~d} y=0$, where $Q:=(-1 / 2,1 / 2)^{d}$ is the open unit cube in $\mathbb{R}^{d}$.

In order to state our first result, we introduce the notion of strong recession function of $f$, which for $(x, A) \in \bar{\Omega} \times \mathbb{R}^{N}$ is defined as

$$
f^{\infty}(x, A):=\lim _{\substack{x^{\prime} \rightarrow x \\ A^{\prime} \rightarrow A \\ t \rightarrow \infty}} \frac{f\left(x^{\prime}, t A^{\prime}\right)}{t}, \quad(x, A) \in \bar{\Omega} \times \mathbb{R}^{N},
$$

provided the limit exists.

Theorem 1.2 (lower semicontinuity). Let $f: \Omega \times \mathbb{R}^{N} \rightarrow[0, \infty)$ be a continuous integrand. Assume that $f$ has linear growth at infinity, that is Lipschitz in its second argument, and that $f(x, \cdot)$ is $\mathscr{A}^{k}$-quasiconvex for all $x \in \Omega$. Further assume that either

(i) $f^{\infty}$ exists in $\Omega \times \mathbb{R}^{N}$, or

(ii) $f^{\infty}$ exists in $\Omega \times \operatorname{span} \Lambda_{\mathscr{A}}$, and there exists a modulus of continuity $\omega:[0, \infty) \rightarrow$ $[0, \infty)$ (increasing, continuous, $\omega(0)=0$ ) such that

$$
|f(x, A)-f(y, A)| \leq \omega(|x-y|)(1+|A|) \quad \text { for all } x, y \in \Omega, A \in \mathbb{R}^{N} \text {. }
$$


Then, the functional

$$
\mathscr{F}[\mu]:=\int_{\Omega} f\left(x, \frac{\mathrm{d} \mu}{\mathrm{d} \mathscr{L}^{d}}(x)\right) \mathrm{d} x+\int_{\Omega} f^{\infty}\left(x, \frac{\mathrm{d} \mu^{s}}{\mathrm{~d}\left|\mu^{s}\right|}(x)\right) \mathrm{d}\left|\mu^{s}\right|(x)
$$

is sequentially weakly* lower semicontinuous on the space

$$
\mathscr{M}\left(\Omega ; \mathbb{R}^{N}\right) \cap \operatorname{ker} \mathscr{A}:=\left\{\mu \in \mathscr{M}\left(\Omega ; \mathbb{R}^{N}\right): \mathscr{A} \mu=0\right\} .
$$

Note that according to (1.6) below, $\mathscr{F}[\mu]$ is well-defined for $\mu \in \mathscr{M}\left(\Omega ; \mathbb{R}^{N}\right) \cap$ $\operatorname{ker} \mathscr{A}$ since the strong recession function is computed only at amplitudes that belong to $\operatorname{span} \Lambda_{\mathscr{A}}$.

Remark 1.3. The $\mathscr{A}^{k}$-quasiconvexity of $f(x, \bullet)$ is not only a sufficient, but also a necessary condition for the sequential weak* lower semicontinuity of $\mathscr{F}$ on $\mathscr{M}\left(\Omega ; \mathbb{R}^{N}\right) \cap$ $\operatorname{ker} \mathscr{A}$. In the case of first-order partial differential operator, the proof of the necessity can be found in [FM99]; the proof in the general case follows by verbatim repeating the same arguments.

Remark 1.4 (asymptotic $\mathscr{A}$-free sequences). The conclusion of Theorem $1.2 \mathrm{ex}$ tends to sequences that are only asymptotically $\mathscr{A}$-free, that is,

$$
\mathscr{F}[\mu] \leq \liminf _{j \rightarrow \infty} \mathscr{F}\left[\mu_{j}\right]
$$

for all sequences $\left(\mu_{j}\right) \subset \mathscr{M}\left(\Omega ; \mathbb{R}^{N}\right)$ such that

$$
\mu_{j} \stackrel{*}{\rightarrow} \mu \text { in } \mathscr{M}\left(\Omega ; \mathbb{R}^{N}\right) \quad \text { and } \quad \mathscr{A} \mu_{j} \rightarrow 0 \text { in } \mathrm{W}^{-k, q}\left(\Omega ; \mathbb{R}^{n}\right)
$$

for some $1<q<d /(d-1)$ if $f(x, \cdot)$ is $\mathscr{A}^{k}$-quasiconvex for all $x \in \Omega$.

Notice that $f^{\infty}$ in (1.3) is a limit and, contrary to $f^{\#}$, it may fail to exist for $A \in\left(\operatorname{span} \Lambda_{\mathscr{A}}\right) \backslash \Lambda_{\mathscr{A}}$ (for $A \in \Lambda_{\mathscr{A}}$ the existence of $f^{\infty}(x, A)$ follows from the $\mathscr{A}^{k}$ quasiconvexity, see Corollary 2.20). If we remove the assumption that $f^{\infty}$ exists for points in the subspace generated by the wave cone $\Lambda_{\mathscr{A}}$, we still have the partial lower semicontinuity result formulated in Theorem 1.6 below (cf. [FLM04]).

Remark 1.5. As special cases of Theorem 1.2 we get, among others, the following well-known results:

(i) For $\mathscr{A}=$ curl, one obtains BV-lower semicontinuity results in the spirit of Ambrosio-Dal Maso [AD92] and Fonseca-Müller [FM93].

(ii) For $\mathscr{A}=$ curl curl, where

$$
\operatorname{curl} \operatorname{curl} \mu:=\left(\sum_{i=1}^{d} \partial_{i k} \mu_{i}^{j}+\partial_{i j} \mu_{i}^{k}-\partial_{j k} \mu_{i}^{i}-\partial_{i i} \mu_{j}^{k}\right)_{j, k=1, \ldots, d}
$$

is the second order operator expressing the Saint-Venant compatibility conditions (see [FM99, Example 3.10(e)]), we re-prove the lower semicontinuity and relaxation theorem in the space of functions of bounded deformation (BD) from [Rin11].

(iii) For first-order operators $\mathscr{A}$, a similar result was proved in [BCMS13].

(iv) Earlier work in this direction is in [FM99, FLM04], but there the singular (concentration) part of the functional was not considered.

Theorem 1.6 (partial lower semicontinuity). Let $f: \Omega \times \mathbb{R}^{N} \rightarrow[0, \infty)$ be a continuous integrand such that $f(x, \cdot)$ is $\mathscr{A}^{k}$-quasiconvex for all $x \in \Omega$. Assume that $f$ 
has linear growth at infinity and is Lipschitz in its second argument, uniformly in $x$. Further, suppose that there exists a modulus of continuity $\omega$ as in (1.4). Then,

$$
\int_{\Omega} f\left(x, \frac{\mathrm{d} \mu}{\mathrm{d} \mathscr{L}^{d}}(x)\right) \mathrm{d} x \leq \liminf _{j \rightarrow \infty} \mathscr{F}^{\#}\left[\mu_{j}\right]
$$

for all sequences $\mu_{j} \stackrel{*}{\rightarrow} \mu$ in $\mathscr{M}\left(\Omega ; \mathbb{R}^{N}\right)$ such that $\mathscr{A} \mu_{j} \rightarrow 0$ in $\mathrm{W}^{-k, q}\left(\Omega ; \mathbb{R}^{N}\right)$. Here,

$$
\mathscr{F}^{\#}[\mu]:=\int_{\Omega} f\left(x, \frac{\mathrm{d} \mu}{\mathrm{d} \mathscr{L}^{d}}(x)\right) \mathrm{d} x+\int_{\Omega} f^{\#}\left(x, \frac{\mathrm{d} \mu^{s}}{\mathrm{~d}\left|\mu^{s}\right|}(x)\right) \mathrm{d}\left|\mu^{s}\right|(x),
$$

and $1<q<d /(d-1)$.

If we dispense with the assumption of $\mathscr{A}^{k}$-quasiconvexity on the integrand, we have the following two relaxation results:

Theorem 1.7 (relaxation). Let $f: \Omega \times \mathbb{R}^{N} \rightarrow[0, \infty)$ be a continuous integrand that is Lipschitz in its second argument, uniformly in $x$. Assume also that $f$ has linear growth at infinity (in its second argument) and is such that there exists a modulus of continuity $\omega$ as in (1.4). Further, suppose that $\mathscr{A}$ is a homogeneous PDE operator and that the strong recession function

$$
f^{\infty}(x, A) \text { exists for all }(x, A) \in \Omega \times \operatorname{span} \Lambda_{\mathscr{A}} .
$$

Then, for the functional

$$
\mathscr{G}[u]:=\int_{\Omega} f(x, u(x)) \mathrm{d} x, \quad u \in \mathrm{L}^{1}\left(\Omega ; \mathbb{R}^{N}\right),
$$

the (sequentially) weakly* lower semicontinuous envelope of $\mathscr{G}$, defined to be

$$
\begin{gathered}
\overline{\mathscr{G}}[\mu]:=\inf \left\{\liminf _{j \rightarrow \infty} \mathscr{G}\left[u_{j}\right]:\left(u_{j}\right) \subset \mathrm{L}^{1}\left(\Omega ; \mathbb{R}^{N}\right), u_{j} \mathscr{L}^{d} \stackrel{*}{\rightarrow} \mu \text { in } \mathscr{M}\left(\Omega ; \mathbb{R}^{N}\right)\right. \\
\text { and } \left.\mathscr{A} u_{j} \rightarrow 0 \text { in } \mathrm{W}^{-k, q}\right\},
\end{gathered}
$$

where $\mu \in \mathscr{M}\left(\Omega ; \mathbb{R}^{N}\right) \cap \operatorname{ker} \mathscr{A}$ and $1<q<d /(d-1)$, is given by

$$
\overline{\mathscr{G}}[\mu]=\int_{\Omega} Q_{\mathscr{A}} f\left(x, \frac{\mathrm{d} \mu}{\mathrm{d} \mathscr{L}^{d}}(x)\right) \mathrm{d} x+\int_{\Omega}\left(Q_{\mathscr{A}} f\right)^{\#}\left(x, \frac{\mathrm{d} \mu^{s}}{\mathrm{~d}\left|\mu^{s}\right|}(x)\right) \mathrm{d}\left|\mu^{s}\right|(x) .
$$

Here, $Q_{\mathscr{A}} f(x, \cdot)$ denotes the $\mathscr{A}$-quasiconvex envelope of $f(x, \cdot)$ with respect to the second argument (see Definition 2.17 below).

If we want to find the relaxation in the space $\mathscr{M}\left(\Omega ; \mathbb{R}^{N}\right) \cap \operatorname{ker} \mathscr{A}$ we need to assume that $\mathrm{L}^{1}\left(\Omega ; \mathbb{R}^{N}\right) \cap \operatorname{ker} \mathscr{A}$ is dense in $\mathscr{M}\left(\Omega ; \mathbb{R}^{N}\right) \cap \operatorname{ker} \mathscr{A}$ with respect to a finer topology than the natural weak* topology (in this context also see [AR16]).

Theorem 1.8. Let $f: \Omega \times \mathbb{R}^{N} \rightarrow[0, \infty)$ be a continuous integrand that is Lipschitz in its second argument, uniformly in $x$. Assume also that $f$ has linear growth at infinity (in its second argument) and is such that there exists a modulus of continuity $\omega$ as in (1.4). Further, suppose that $\mathscr{A}$ is a homogeneous PDE operator, that the strong recession function

$$
f^{\infty}(x, A) \text { exists for all }(x, A) \in \Omega \times \operatorname{span} \Lambda_{\mathscr{A}},
$$

and that for all $\mu \in \mathscr{M}\left(\Omega ; \mathbb{R}^{N}\right) \cap \operatorname{ker} \mathscr{A}$ there exists a sequence $\left(u_{j}\right) \subset \mathrm{L}^{1}\left(\Omega ; \mathbb{R}^{N}\right) \cap$ ker $\mathscr{A}$ such that

$$
u_{j} \mathscr{L}^{d} \stackrel{*}{\rightarrow} \mu \quad \text { in } \mathscr{M}\left(\Omega ; \mathbb{R}^{N}\right) \quad \text { and } \quad\left\langle u_{j} \mathscr{L}^{d}\right\rangle(\Omega) \rightarrow\langle\mu\rangle(\Omega),
$$


where $\langle\cdot\rangle$ is the area functional defined in (2.2). Then, for the functional

$$
\mathscr{G}[u]:=\int_{\Omega} f(x, u(x)) \mathrm{d} x, \quad u \in \mathrm{L}^{1}\left(\Omega ; \mathbb{R}^{N}\right) \cap \operatorname{ker} \mathscr{A},
$$

the weakly* lower semicontinuous envelope of $\mathscr{G}$, defined to be

$\overline{\mathscr{G}}[\mu]:=\inf \left\{\liminf _{j \rightarrow \infty} \mathscr{G}\left[u_{j}\right]:\left(u_{j}\right) \subset \mathrm{L}^{1}\left(\Omega ; \mathbb{R}^{N}\right) \cap \operatorname{ker} \mathscr{A}, u_{j} \mathscr{L}^{d} \stackrel{*}{\rightarrow} \mu\right.$ in $\left.\mathscr{M}\left(\Omega ; \mathbb{R}^{N}\right)\right\}$,

is given by

$$
\overline{\mathscr{G}}[\mu]=\int_{\Omega} Q_{\mathscr{A}} f\left(x, \frac{\mathrm{d} \mu}{\mathrm{d} \mathscr{L}^{d}}(x)\right) \mathrm{d} x+\int_{\Omega}\left(Q_{\mathscr{A}} f\right)^{\#}\left(x, \frac{\mathrm{d} \mu^{s}}{\mathrm{~d}\left|\mu^{s}\right|}(x)\right) \mathrm{d}\left|\mu^{s}\right|(x) .
$$

Remark 1.9 (density assumptions). Condition (1.5) is automatically fulfilled in the following cases:

(i) For $\mathscr{A}=$ curl, the approximation property (for general domains) is proved in the appendix of [KR10a] (also see Lemma B.1 of [Bil03] for Lipschitz domains). The same argument further shows the area-strict approximation property in the BD-case (also see Lemma 2.2 in [BFT00] for a result which covers the strict convergence).

(ii) If $\Omega$ is a strictly star-shaped domain, i.e., there exists $x_{0} \in \Omega$ such that

$$
\overline{\left(\Omega-x_{0}\right)} \subset t\left(\Omega-x_{0}\right) \quad \text { for all } t>1,
$$

then (1.5) holds for every homogeneous operator $\mathscr{A}$. Indeed, for $t>1$ we can consider the dilation of $\mu$ defined on $t\left(\Omega-x_{0}\right)$ and then mollify it at a sufficiently small scale. We refer for instance to [Mül87] for details.

As a consequence of Theorem 1.8 and of Remark 1.9 we explicitly state the following corollary, which extends the lower semicontinuity result of [Rin11] into a full relaxation result. The only other relaxation result in this direction, albeit for special functions of bounded deformation, seems to be in [BFT00]; other results in this area are discussed in [Rin11] and the references therein.

Corollary 1.10. Let $f: \Omega \times \mathbb{R}_{\text {sym }}^{d \times d} \rightarrow[0, \infty)$ be a continuous integrand, uniformly Lipschitz in the second argument, with linear growth at infinity, and such that there exists a modulus of continuity $\omega$ as in (1.4). Further, suppose that the strong recession function

$$
f^{\infty}(x, A) \text { exists for all }(x, A) \in \Omega \times \mathbb{R}_{\mathrm{sym}}^{d \times d} .
$$

Consider the functional

$$
\mathscr{G}[u]:=\int_{\Omega} f(x, \mathscr{E} u(x)) \mathrm{d} x,
$$

defined for $u \in \operatorname{LD}(\Omega):=\left\{u \in \operatorname{BD}(\Omega): E^{s} u=0\right\}$, where $E u:=\left(D u+D u^{T}\right) / 2 \in$ $\mathscr{M}\left(\Omega ; \mathbb{R}_{\mathrm{sym}}^{d \times d}\right)$ is the symmetrized distributional derivative of $u \in \mathrm{BD}(\Omega)$ and where

$$
E u=\mathscr{E} u \mathscr{L}^{d}\left\llcorner\Omega+E^{s} u\right.
$$

is its Radon-Nikodým decomposition with respect to $\mathscr{L}^{d}$.

Then, the lower semicontinuous envelope of $\mathscr{G}$ with respect to weak*-convergence in $\mathrm{BD}(\Omega)$ is given by the functional

$$
\overline{\mathscr{G}}[u]:=\int_{\Omega} S Q f(x, \mathscr{E} u(x)) \mathrm{d} x+\int_{\Omega}(S Q f)^{\#}\left(x, \frac{\mathrm{d} E^{s} u}{\mathrm{~d}\left|E^{s} u\right|}(x)\right) \mathrm{d}\left|E^{s} u\right|(x),
$$


where $S Q f$ denotes the symmetric-quasiconvex envelope of $f$ with respect to the second argument (i.e., the curl curl-quasiconvex envelope of $f(x, \cdot)$ in the sense of Definition 2.17).

Our proofs are based on new tools to study singularities in PDE-constrained measures. Concretely, we exploit the recent developments on the structure of $\mathscr{A}$-free measures obtained in [DR16b]. We remark that the study of the singular part - up to now the most complicated argument in the proof - now only requires a fairly straightforward (classical) convexity argument. More precisely, the main theorem of [KK16] establishes that the restriction of $f^{\#}$ to the linear space spanned by the wave cone is in fact convex at all points of $\Lambda_{\mathscr{A}}$ (in the sense that a supporting hyperplane exists). By [DR16b],

$$
\frac{\mathrm{d} \mu^{s}}{\mathrm{~d}\left|\mu^{s}\right|}(x) \in \Lambda_{\mathscr{A}} \quad \text { for }\left|\mu^{s}\right| \text {-a.e. } x \in \Omega .
$$

Thus, combining these two assertions, we gain classical convexity for $f^{\#}$ at singular points, which can be exploited via the theory of generalized Young measures developed in [DM87, AB97, KR10a].

Remark 1.11 (different notions of recession function). Note that both in Theorem 1.2 and Theorem 1.7 the existence of the strong recession function $f^{\infty}$ is assumed, in contrast with the results in [AD92, FM93, BCMS13] where this is not imposed.

The need for this assumption comes from the use of Young measure techniques which seem to be better suited to deal with the singular part of the measure, as we already discussed above. In the aforementioned references a direct blow up approach is instead performed and this allows to deal directly with the functional in (1.1). The blow-up techniques, however, rely strongly on the fact that $\mathscr{A}$ is a homogeneous first-order operator. Indeed, it is not hard to check that for all "elementary" $\mathscr{A}$-free measures of the form

$$
\mu=P_{0} \lambda, \quad \text { where } \quad P_{0} \in \Lambda_{\mathscr{A}}, \lambda \in \mathscr{M}^{+}\left(\mathbb{R}^{d}\right),
$$

the scalar measure $\lambda$ is necessarily translation invariant along orthogonal directions to the characteristic set

$$
\Xi\left(P_{0}\right):=\left\{\xi \in \mathbb{R}^{d}: P_{0} \in \operatorname{ker} \mathbb{A}(\xi)\right\},
$$

which turns out to be a subspace of $\mathbb{R}^{d}$ whenever $\mathscr{A}$ is a first-order operator. The subspace structure and the aforementioned translation invariance is then used to perform homogenization-type arguments. Due to the lack of linearity of the map

$$
\xi \mapsto \mathbb{A}^{k}(\xi) \text { for } k>1,
$$

the structure of elementary $\mathscr{A}$-free measures for general operators is more complicated and not yet fully understood (see however [Rin11, DR16a] for the case $\mathscr{A}=$ curl curl). This prevents, at the moment, the use of "pure" blow-up techniques and forces us to pass through the combination of the results of [DR16b, KK16] with the Young measure approach.

This paper is organized as follows: First, in Section 2, we introduce all the necessary notation and prove auxiliary results. Then, in Section 3, we establish the central Jensen-type inequalities, which immediately yield the proof of Theorems 1.2 and 1.6 in Section 4. The proofs of Theorems 1.7 and 1.8 are given in Section 5. 
Acknowledgments. A. A.-R. is supported by a scholarship from the Hausdorff Center of Mathematics and the University of Bonn through a DFG grant; the research conducted in this paper forms part of his Ph.D. thesis at the University of Bonn. G. D. P. is supported by the MIUR SIR-grant "Geometric Variational Problems" (RBSI14RVEZ). F. R. acknowledges the support from an EPSRC Research Fellowship on "Singularities in Nonlinear PDEs" (EP/L018934/1).

The authors would like to thank the anonymous referee for her/his careful reading of the manuscript which led to a substantial improvement of the presentation.

\section{NOTATION AND PRELIMINARIES}

We write $\mathscr{M}\left(\Omega ; \mathbb{R}^{N}\right)$ and $\mathscr{M}_{\mathrm{loc}}\left(\Omega ; \mathbb{R}^{N}\right)$ to denote the spaces of bounded Radon measures and Radon measures on $\Omega \subset \mathbb{R}^{d}$ and with values in $\mathbb{R}^{N}$, which are the duals of $\mathrm{C}_{0}\left(\Omega ; \mathbb{R}^{N}\right)$ and $\mathrm{C}_{c}\left(\Omega ; \mathbb{R}^{N}\right)$ respectively. Here, $\mathrm{C}_{0}\left(\Omega ; \mathbb{R}^{N}\right)$ is the completion of $\mathrm{C}_{c}\left(\Omega ; \mathbb{R}^{N}\right)$ with respect to the $\|\cdot\|_{\infty}$-norm, and, in the second case, $\mathrm{C}_{c}\left(\Omega ; \mathbb{R}^{N}\right)$ is understood as the inductive limit of the Banach spaces $\mathrm{C}_{0}\left(K_{m}\right)$ where each $K_{m}$ is a compact subset of $\mathbb{R}^{d}$ and $K_{m} \nearrow \Omega$. The set of probability measures over a locally compact space $X$ shall be denoted by

$$
\mathscr{M}^{1}(X):=\{\mu \in \mathscr{M}(X): \mu \text { is a positive measure, and } \mu(X)=1\} .
$$

We will often make use of the following metrizability principles:

(1) Bounded sets of $\mathscr{M}\left(\Omega ; \mathbb{R}^{N}\right)$ are metrizable in the sense that there exists a metric $d$ which induces the weak* topology, that is,

$$
\sup _{j \in \mathbb{N}}\left|\mu_{j}\right|(\Omega)<\infty \quad \text { and } \quad d\left(\mu_{j}, \mu\right) \rightarrow 0 \quad \Leftrightarrow \quad \mu_{j} \stackrel{*}{\rightarrow} \mu \quad \text { in } \mathscr{M}\left(\Omega ; \mathbb{R}^{N}\right) .
$$

(2) There exists a complete metric $d$ on $\mathscr{M}_{\text {loc }}\left(\Omega ; \mathbb{R}^{N}\right)$. Moreover, convergence with respect to this metric coincides with the weak* convergence of Radon measures (see Remark 14.15 in [Mat95]).

We write the Radon-Nikodým decomposition of a measure $\mu \in \mathscr{M}\left(\Omega ; \mathbb{R}^{N}\right)$ as

$$
\mu=\frac{\mathrm{d} \mu}{\mathrm{d} \mathscr{L}^{d}} \mathscr{L}^{d}\left\llcorner\Omega+\mu^{s}\right.
$$

where $\frac{\mathrm{d} \mu}{\mathrm{d} \mathscr{L}^{d}} \in \mathrm{L}^{1}\left(\Omega ; \mathbb{R}^{N}\right)$ and $\mu^{s} \in \mathscr{M}\left(\Omega ; \mathbb{R}^{N}\right)$ is singular with respect to $\mathscr{L}^{d}$.

2.1. Integrands and Young measures. For $f \in \mathrm{C}\left(\Omega \times \mathbb{R}^{N}\right)$ we define the transformation

$$
(S f)(x, \hat{A}):=(1-|\hat{A}|) f\left(x, \frac{\hat{A}}{1-|\hat{A}|}\right), \quad(x, \hat{A}) \in \bar{\Omega} \times \mathbb{B}^{N},
$$

where $\mathbb{B}^{N}$ denotes the open unit ball in $\mathbb{R}^{N}$. Then, $S f \in \mathrm{C}\left(\Omega \times \mathbb{B}^{N}\right)$. We set

$$
\mathbf{E}\left(\Omega ; \mathbb{R}^{N}\right):=\left\{f \in \mathrm{C}\left(\Omega \times \mathbb{R}^{N}\right): S f \text { extends to } \mathrm{C}\left(\overline{\Omega \times \mathbb{B}^{N}}\right)\right\} .
$$

In particular, all $f \in \mathbf{E}\left(\Omega ; \mathbb{R}^{N}\right)$ have linear growth at infinity, i.e., there exists a positive constant $M$ such that $|f(x, A)| \leq M(1+|A|)$ for all $x \in \Omega$ and all $A \in \mathbb{R}^{N}$. With the norm

$$
\|f\|_{\mathbf{E}\left(\Omega ; \mathbb{R}^{N}\right)}:=\|S f\|_{\infty}, \quad f \in \mathbf{E}\left(\Omega ; \mathbb{R}^{N}\right),
$$


the space $\mathbf{E}\left(\Omega ; \mathbb{R}^{N}\right)$ turns out to be a Banach space. Also, by definition, for each $f \in \mathbf{E}\left(\Omega ; \mathbb{R}^{N}\right)$ the limit

$$
f^{\infty}(x, A):=\lim _{\substack{x^{\prime} \rightarrow x \\ A^{\prime} \rightarrow A \\ t \rightarrow \infty}} \frac{f\left(x^{\prime}, t A^{\prime}\right)}{t}, \quad(x, A) \in \bar{\Omega} \times \mathbb{R}^{N},
$$

exists and defines a positively 1-homogeneous function called the strong recession function of $f$. Even if one drops the dependence on $x$, the recession function $h^{\infty}$ might not exist for $h \in \mathrm{C}\left(\mathbb{R}^{N}\right)$. Instead, one can always define the upper and lower recession functions

$$
\begin{gathered}
f^{\#}(x, A):=\limsup _{\substack{x^{\prime} \rightarrow x \\
A^{\prime} \rightarrow A \\
t \rightarrow \infty}} \frac{f\left(x^{\prime}, t A^{\prime}\right)}{t}, \\
f_{\#}(x, A):=\liminf _{\substack{x^{\prime} \rightarrow x \\
A^{\prime} \rightarrow A \\
t \rightarrow \infty}} \frac{f\left(x^{\prime}, t A^{\prime}\right)}{t},
\end{gathered}
$$

which again can be seen to be positively 1-homogeneous. If $f$ is $x$-uniformly Lipschitz continuous in the $A$-variable and there exists a modulus of continuity $\omega:[0, \infty) \rightarrow$ $[0, \infty)$ (increasing, continuous, and $\omega(0)=0$ ) such that

$$
|f(x, A)-f(y, A)| \leq \omega(|x-y|)(1+|A|), \quad x, y \in \Omega, A \in \mathbb{R}^{N},
$$

then the definitions of $f^{\infty}, f^{\#}$, and $f_{\#}$ simplify to

$$
\begin{aligned}
f^{\infty}(x, A) & :=\lim _{t \rightarrow \infty} \frac{f(x, t A)}{t}, \\
f^{\#}(x, A) & :=\limsup _{t \rightarrow \infty} \frac{f(x, t A)}{t}, \\
f_{\#}(x, A) & :=\liminf _{t \rightarrow \infty} \frac{f(x, t A)}{t} .
\end{aligned}
$$

A natural action of $\mathbf{E}\left(\Omega ; \mathbb{R}^{N}\right)$ on the space $\mathscr{M}\left(\Omega ; \mathbb{R}^{N}\right)$ is given by

$$
\mu \mapsto \int_{\Omega} f\left(x, \frac{\mathrm{d} \mu}{\mathrm{d} \mathscr{L}^{N}}(x)\right) \mathrm{d} x+\int_{\Omega} f^{\infty}\left(x, \frac{\mathrm{d} \mu^{s}}{\mathrm{~d}\left|\mu^{s}\right|}(x)\right) \mathrm{d}\left|\mu^{s}\right|(x) .
$$

In particular, for $f(x, A)=\sqrt{1+|A|^{2}} \in \mathbf{E}\left(\Omega ; \mathbb{R}^{N}\right)$, for which $f^{\infty}(A)=|A|$, we define the area functional

$$
\langle\mu\rangle(\Omega):=\int_{\Omega} \sqrt{1+\left|\frac{\mathrm{d} \mu}{\mathrm{d} \mathscr{L}^{N}}\right|^{2}} \mathrm{~d} x+\left|\mu^{s}\right|(\Omega), \quad \mu \in \mathscr{M}\left(\Omega ; \mathbb{R}^{N}\right) .
$$

In addition to the well-known weak* convergence of measures, we say that a sequence $\left(\mu_{j}\right)$ converges area-strictly to $\mu$ in $\mathscr{M}\left(\Omega ; \mathbb{R}^{N}\right)$ if

$$
\mu_{j} \stackrel{*}{\rightarrow} \mu \text { in } \mathscr{M}\left(\Omega ; \mathbb{R}^{N}\right) \quad \text { and } \quad\left\langle\mu_{j}\right\rangle(\Omega) \rightarrow\langle\mu\rangle(\Omega) .
$$

This notion of convergence turns out to be stronger than the conventional strict convergence of measures, which means that

$$
\mu_{j} \stackrel{*}{\rightarrow} \mu \quad \text { in } \mathscr{M}\left(\Omega ; \mathbb{R}^{N}\right) \quad \text { and } \quad\left|\mu_{j}\right|(\Omega) \rightarrow|\mu|(\Omega) .
$$

Indeed, the area-strict convergence, as opposed to the usual strict convergence, prohibits oscillations of the absolutely continuous part. The meaning of area-strict convergence becomes clear when considering the following version of Reshetnyak's continuity theorem, which entails that the topology generated by area-strict convergence 
is the coarsest topology under which the natural action of $\mathbf{E}\left(\Omega ; \mathbb{R}^{N}\right)$ on $\mathscr{M}\left(\Omega ; \mathbb{R}^{N}\right)$ is continuous.

Theorem 2.1 (Theorem 5 in [KR10b]). For every integrand $f \in \mathbf{E}\left(\Omega ; \mathbb{R}^{N}\right)$, the functional

$$
\mu \mapsto \int_{\Omega} f\left(x, \frac{\mathrm{d} \mu}{\mathrm{d} \mathscr{L}^{N}}(x)\right) \mathrm{d} x+\int_{\Omega} f^{\infty}\left(x, \frac{\mathrm{d} \mu^{s}}{\mathrm{~d}\left|\mu^{s}\right|}(x)\right) \mathrm{d}\left|\mu^{s}\right|(x),
$$

is area-strictly continuous on $\mathscr{M}\left(\Omega ; \mathbb{R}^{N}\right)$.

Remark 2.2. Notice that if $\mu \in \mathscr{M}\left(\mathbb{R}^{d} ; \mathbb{R}^{N}\right)$, then $\mu_{\varepsilon} \rightarrow \mu$ area-strictly, where $\mu_{\varepsilon}$ is the mollification of $\mu$ with a family of standard convolution kernels, $\mu_{\varepsilon}:=\mu * \rho_{\varepsilon}$ and $\rho_{\varepsilon}(x):=\varepsilon^{-d} \rho(x / \varepsilon)$ for $\rho \in \mathrm{C}_{c}^{\infty}\left(B_{1}\right)$ a positive and even function satisfying $\int \rho \mathrm{d} x=$ 1.

Generalized Young measures form a set of dual objects to the integrands in $\mathbf{E}\left(\Omega ; \mathbb{R}^{N}\right)$. We recall briefly some aspects of this theory, which was introduced by DiPerna and Majda in [DM87] and later extended in [AB97, KR10a].

Definition 2.3 (generalized Young measure). A generalized Young measure, parameterized by an open set $\Omega \subset \mathbb{R}^{d}$, and with values in $\mathbb{R}^{N}$, is a triple $v=\left(v_{x}, \lambda_{v}, v_{x}^{\infty}\right)$, where

(i) $\left(v_{x}\right)_{x \in \Omega} \subset \mathscr{M}^{1}\left(\mathbb{R}^{N}\right)$ is a parameterized family of probability measures on $\mathbb{R}^{N}$,

(ii) $\lambda_{v} \in \mathscr{M}_{+}(\bar{\Omega})$ is a positive finite Radon measure on $\bar{\Omega}$, and

(iii) $\left(v_{x}^{\infty}\right)_{x \in \bar{\Omega}} \subset \mathscr{M}^{1}\left(\mathbb{S}^{N-1}\right)$ is a parametrized family of probability measures on the unit sphere $\mathbb{S}^{N-1}$.

Additionally, we require that

(iv) the map $x \mapsto v_{x}$ is weakly* measurable with respect to $\mathscr{L}^{d}$,

(v) the map $x \mapsto v_{x}^{\infty}$ is weakly* measurable with respect to $\lambda_{v}$, and

(vi) $x \mapsto\left\langle|\cdot|, v_{x}\right\rangle \in \mathrm{L}^{1}(\Omega)$.

The set of all such Young measures is denoted by $\mathbf{Y}\left(\Omega ; \mathbb{R}^{N}\right)$.

Similarly we say that $v \in \mathbf{Y}_{\mathrm{loc}}\left(\Omega ; \mathbb{R}^{N}\right)$ if $v \in \mathbf{Y}\left(E ; \mathbb{R}^{N}\right)$ for all open $E \Subset \Omega$.

Here, weak* measurability means that the functions $x \mapsto\left\langle f(x, \cdot), v_{x}\right\rangle$ (respectively $\left.x \mapsto\left\langle f^{\infty}(x, \bullet), v_{x}^{\infty}\right\rangle\right)$ are Lebesgue-measurable (respectively $\lambda_{v}$-measurable) for all Carathéodory integrands $f: \Omega \times \mathbb{R}^{N} \rightarrow \mathbb{R}$ (measurable in their first argument and continuous in their second argument).

For an integrand $f \in \mathbf{E}\left(\Omega ; \mathbb{R}^{N}\right)$ and a Young measure $v \in \mathbf{Y}\left(\Omega ; \mathbb{R}^{N}\right)$, we define the duality paring between $f$ and $v$ as follows:

$$
\left\langle\langle f, v\rangle:=\int_{\Omega}\left\langle f(x, \cdot), v_{x}\right\rangle \mathrm{d} x+\int_{\bar{\Omega}}\left\langle f^{\infty}(x, \cdot), v_{x}^{\infty}\right\rangle \mathrm{d} \lambda_{v}(x) .\right.
$$

In many cases it will be sufficient to work with functions $f \in \mathbf{E}\left(\Omega ; \mathbb{R}^{N}\right)$ that are Lipschitz continuous. The following density lemma can be found in [KR10a, Lemma 3].

Lemma 2.4. There exists a countable set of functions $\left\{f_{m}\right\}=\left\{\varphi_{m} \otimes h_{m} \in \mathrm{C}(\bar{\Omega}) \times\right.$ $\left.\mathrm{C}\left(\mathbb{R}^{N}\right): m \in \mathbb{N}\right\} \subset \mathbf{E}\left(\Omega ; \mathbb{R}^{N}\right)$ such that for two Young measures $v_{1}, v_{2} \in \mathbf{Y}\left(\Omega ; \mathbb{R}^{N}\right)$ 
the implication

$$
\left\langle\left\langle f_{m}, v_{1}\right\rangle\right\rangle=\left\langle\left\langle f_{m}, v_{2}\right\rangle \quad \forall m \in \mathbb{N} \quad \Longrightarrow \quad v_{1}=v_{2}\right.
$$

holds. Moreover, all the $h_{m}$ can be chosen to be Lipschitz continuous and all the $\varphi_{m}$ can be chosen to be non-negative.

Since $\mathbf{Y}\left(\Omega ; \mathbb{R}^{N}\right)$ is contained in the dual space of $\mathbf{E}\left(\Omega ; \mathbb{R}^{N}\right)$ via the duality pairing $\langle\cdot \cdot \cdot \cdot\rangle\rangle$, we say that a sequence of Young measures $\left(v_{j}\right) \subset \mathbf{Y}\left(\Omega ; \mathbb{R}^{N}\right)$ converges weakly* to $v \in \mathbf{Y}\left(\Omega ; \mathbb{R}^{N}\right)$, in symbols $v_{j} \stackrel{*}{\rightarrow} v$, if

$$
\left\langle\langle f , v _ { j } \rangle \rightarrow \left\langle\langle f, v\rangle \quad \text { for all } f \in \mathbf{E}\left(\Omega ; \mathbb{R}^{N}\right) .\right.\right.
$$

Fundamental for all Young measure theory is the following compactness result, see [KR10a, Section 3.1] for a proof.

Lemma 2.5 (compactness). Let $\left(v_{j}\right) \subset \mathbf{Y}\left(\Omega ; \mathbb{R}^{N}\right)$ be a sequence of Young measures satisfying

(i) the functions $x \mapsto\left\langle|\cdot|, v_{j}\right\rangle$ are uniformly bounded in $\mathrm{L}^{1}(\Omega)$,

(ii) $\sup _{j} \lambda_{v_{j}}(\bar{\Omega})<\infty$.

Then, there exists a subsequence (not relabeled) and $v \in \mathbf{Y}\left(\Omega ; \mathbb{R}^{N}\right)$ such that $v_{j} \stackrel{*}{\rightarrow} v$ in $\mathbf{Y}\left(\Omega ; \mathbb{R}^{N}\right)$.

The Radon-Nikodým decomposition (2.1) induces a natural embedding of $\mathscr{M}\left(\Omega ; \mathbb{R}^{N}\right)$ into $\mathbf{Y}\left(\Omega ; \mathbb{R}^{N}\right)$ via the identification $\mu \mapsto \delta[\mu]$, where

$$
(\delta[\mu])_{x}:=\delta_{\frac{\mathrm{d} \mu}{\mathrm{d} \mathscr{L}^{d}}(x)}, \quad \lambda_{\delta[\mu]}:=\left|\mu^{s}\right|, \quad(\delta[\mu])_{x}^{\infty}:=\delta_{\frac{\mathrm{d} \mu^{s}}{\mathrm{~d} \mid \mu^{s}}(x)} .
$$

In this sense, we say that the sequence of measures $\left(\mu_{j}\right)$ generates the Young measure $v$ if $\delta\left[\mu_{h}\right] \stackrel{*}{\rightarrow} v$ in $\mathbf{Y}\left(\Omega ; \mathbb{R}^{N}\right)$; we write

$$
\mu_{j} \stackrel{\mathbf{Y}}{\rightarrow} v .
$$

The barycenter of a Young measure $v \in \mathbf{Y}\left(\Omega ; \mathbb{R}^{N}\right)$ is defined as the measure

$$
[v]:=\left\langle\mathrm{id}, v_{x}\right\rangle \mathscr{L}^{d}\left\llcorner\Omega+\left\langle\mathrm{id}, v_{x}^{\infty}\right\rangle \lambda_{v} \in \mathscr{M}\left(\bar{\Omega} ; \mathbb{R}^{N}\right) .\right.
$$

Using the notation above it is clear that for $\left(\mu_{j}\right) \subset \mathscr{M}\left(\Omega ; \mathbb{R}^{N}\right)$ it holds that $\mu_{j}{ }^{*}[v]$, as measures on $\bar{\Omega}$, if $\mu_{j} \stackrel{\mathrm{Y}}{\rightarrow} v$.

Remark 2.6. For a sequence $\left(\mu_{j}\right) \subset \mathscr{M}\left(\Omega ; \mathbb{R}^{N}\right)$ that area-strictly converges to some limit $\mu \in \mathscr{M}\left(\Omega ; \mathbb{R}^{N}\right)$, it is relatively easy to characterize the (unique) Young measure it generates. Indeed, an immediate consequence of the Separation Lemma 2.4 and Theorem 2.1 is that

$$
\mu_{j} \rightarrow \mu \text { area-strictly in } \Omega \quad \Longleftrightarrow \quad \mu_{j} \stackrel{\mathbf{Y}}{\rightarrow} \delta[\mu] \in \mathbf{Y}\left(\Omega ; \mathbb{R}^{N}\right) .
$$

Young measures generated by means of periodic homogenization can be easily computed, see Lemma A.1 in [BM84].

Lemma 2.7 (oscillation measures). Let $1 \leq p<\infty$ and let $w \in \mathrm{L}_{\mathrm{loc}}^{p}\left(\mathbb{R}^{d} ; \mathbb{R}^{N}\right)$ be a $Q$-periodic function and let $m \in \mathbb{N}$. Define the $(Q / m)$-periodic functions $w_{m}(x):=$ $w(m x)$. Then,

$$
w_{m} \rightarrow \bar{w}(x):=\int_{Q} w(y) \mathrm{d} y
$$


in $\mathrm{L}_{\mathrm{loc}}^{p}\left(\mathbb{R}^{d} ; \mathbb{R}^{N}\right)$.

In particular, the sequence $\left(w_{m}\right) \subset \mathrm{L}_{\mathrm{loc}}^{1}\left(\mathbb{R}^{d} ; \mathbb{R}^{N}\right)$ generates the homogeneous (local) Young measure $v=\left(\overline{\delta_{w}}, 0, \cdot\right) \in \mathbf{Y}_{\mathrm{loc}}\left(\mathbb{R}^{d} ; \mathbb{R}^{N}\right)$ (since $\lambda_{v}$ is the zero measure, the component $v_{x}^{\infty}$ can be occupied by any parameterized family of probability measures in $\mathscr{M}^{1}\left(\mathbb{S}^{N-1}\right)$ ), where

$$
\left\langle h, \overline{\delta_{w}}\right\rangle:=\int_{Q} h(w(y)) \mathrm{d} y \quad \text { for all } h \in \mathrm{C}\left(\mathbb{R}^{d}\right) \text { with linear growth at infinity. }
$$

In some cases it will be necessary to determine the smallest linear space containing the support of a Young measure. With this aim in mind, we state the following version of Theorem 2.5 in [AB97]:

Lemma 2.8. Let $\left(u_{j}\right)$ be a sequence in $\mathrm{L}^{1}\left(\Omega ; \mathbb{R}^{N}\right)$ generating a Young measure $v \in \mathbf{Y}\left(\Omega ; \mathbb{R}^{N}\right)$ and let $V$ be a subspace of $\mathbb{R}^{N}$ such that $u_{j}(x) \in V$ for $\mathscr{L}^{d}$-a.e. $x \in \Omega$. Then,

(i) $\operatorname{supp} v_{x} \subset V$ for $\mathscr{L}^{d}$-a.e. $x \in \Omega$,

(ii) $\operatorname{supp} v_{x}^{\infty} \subset V \cap \mathbb{S}^{N-1}$ for $\lambda_{v}$-a.e. $x \in \Omega$.

Finally, we have the following approximation lemma, see [AB97, Lemma 2.3] for a proof.

Lemma 2.9. Let $f: \Omega \times \mathbb{R}^{N} \rightarrow \mathbb{R}$ be an upper semicontinuous integrand with linear growth at infinity. Then, there exists a decreasing sequence $\left(f_{m}\right) \subset \mathbf{E}\left(\Omega ; \mathbb{R}^{N}\right)$ such that

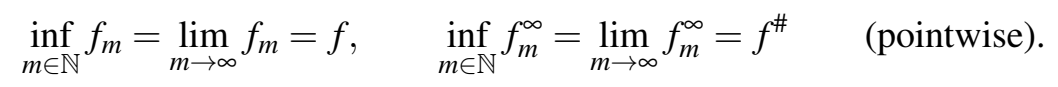

Furthermore, the linear growth constants of the $f_{m}$ 's can be chosen to be bounded by the linear growth constant of $f$.

By approximation, we thus get:

Corollary 2.10. Let $f: \Omega \times \mathbb{R}^{N} \rightarrow \mathbb{R}$ be an upper semicontinuous Borel integrand. Then the functional

$$
v \mapsto \int_{\Omega}\left\langle f(x, \cdot), v_{x}\right\rangle \mathrm{d} x+\int_{\bar{\Omega}}\left\langle f^{\#}(x, \cdot), v_{x}^{\infty}\right\rangle \mathrm{d} \lambda_{v}(x)
$$

is sequentially weakly* upper semicontinuous on $\mathbf{Y}\left(\Omega ; \mathbb{R}^{N}\right)$.

Similarly, if $f: \Omega \times \mathbb{R}^{N} \rightarrow \mathbb{R}$ is a lower semicontinuous Borel integrand, then the functional

$$
v \mapsto \int_{\Omega}\left\langle f(x, \cdot), v_{x}\right\rangle \mathrm{d} x+\int_{\bar{\Omega}}\left\langle f_{\#}(x, \cdot), v_{x}^{\infty}\right\rangle \mathrm{d} \lambda_{v}(x)
$$

is sequentially weakly* lower semicontinuous on $\mathbf{Y}\left(\Omega ; \mathbb{R}^{N}\right)$.

2.2. Tangent measures. In this section we recall the notion of tangent measures, as introduced by Preiss [Pre87] (with the exception that we always include the zero measure as a tangent measure).

Let $\mu \in \mathscr{M}\left(\Omega ; \mathbb{R}^{N}\right)$ and consider the map $T^{\left(x_{0}, r\right)}(x):=\left(x-x_{0}\right) / r$, which blows up $B_{r}\left(x_{0}\right)$, the open ball around $x_{0} \in \Omega$ with radius $r>0$, into the open unit ball $B_{1}$. The push-forward of $\mu$ under $T^{\left(x_{0}, r\right)}$ is given by the measure

$$
T_{\#}^{\left(x_{0}, r\right)} \mu(B):=\mu\left(x_{0}+r B\right), \quad B \subset r^{-1}\left(\Omega-x_{0}\right) \text { a Borel set. }
$$


We say that $v$ is a tangent measure to $\mu$ at a point $x_{0} \in \mathbb{R}^{d}$ if there exist sequences $r_{m}>0, c_{m}>0$ with $r_{m} \downarrow 0$ such that

$$
c_{m} T_{\#}^{\left(x_{0}, r_{m}\right)} \mu \stackrel{*}{\rightarrow} v \quad \text { in } \mathscr{M}_{\mathrm{loc}}\left(\mathbb{R}^{d} ; \mathbb{R}^{N}\right) .
$$

The set of all such tangent measures is denoted by $\operatorname{Tan}\left(\mu, x_{0}\right)$ and the sequence $c_{m} T_{\#}^{\left(x_{0}, r_{m}\right)} \mu$ is called a blow-up sequence. Using the canonical zero extension that maps the space $\mathscr{M}\left(\Omega ; \mathbb{R}^{N}\right)$ into the space $\mathscr{M}\left(\mathbb{R}^{d} ; \mathbb{R}^{N}\right)$ we may use most of the results contained in the general theory for tangent measures when dealing with tangent measures defined on smaller domains.

Since we will frequently restrict tangent measures to the $d$-dimensional unit cube $Q:=(-1 / 2,1 / 2)^{d}$, we set

$$
\operatorname{Tan}_{Q}\left(\mu, x_{0}\right):=\left\{\sigma\left\llcorner\bar{Q}: \sigma \in \operatorname{Tan}\left(\mu, x_{0}\right)\right\} .\right.
$$

One can show (see Remark 14.4 in [Mat95]) that for any non-zero $\sigma \in \operatorname{Tan}\left(\mu, x_{0}\right)$ it is always possible to choose the scaling constants $c_{m}>0$ in the blow-up sequence to be

$$
c_{m}:=c \mu\left(x_{0}+r_{m} \bar{U}\right)^{-1}
$$

for any open and bounded set $U \subset \mathbb{R}^{d}$ containing the origin and with the property that $\sigma(U)>0$, for some positive constant $c=c(U)$ (this may involve passing to a subsequence).

A special property of tangent measures is that at $|\mu|$-almost every $x_{0} \in \mathbb{R}^{d}$ it holds that

$$
\sigma=\mathrm{w}^{*}-\lim _{m \rightarrow \infty} c_{m} T_{\#}^{\left(x_{0}, r_{m}\right)} \mu \Longleftrightarrow|\sigma|=\mathrm{w}^{*}-\lim _{m \rightarrow \infty} c_{m} T_{\#}^{\left(x_{0}, r_{m}\right)}|\mu|,
$$

where the weak* limits are to be understood in the spaces $\mathscr{M}_{\mathrm{loc}}\left(\mathbb{R}^{d} ; \mathbb{R}^{N}\right)$ and $\mathscr{M}_{\mathrm{loc}}^{+}\left(\mathbb{R}^{d}\right)$, respectively. A proof of this fact can be found in Theorem 2.44 of [AFP00]. In particular, this implies

$$
\operatorname{Tan}\left(\mu, x_{0}\right)=\frac{\mathrm{d} \mu}{\mathrm{d}|\mu|}\left(x_{0}\right) \cdot \operatorname{Tan}\left(|\mu|, x_{0}\right) \quad \text { for }|\mu| \text {-almost every } x_{0} \in \mathbb{R}^{d} .
$$

If $\mu, \lambda \in \mathscr{M}_{\mathrm{loc}}^{+}\left(\mathbb{R}^{d}\right)$ are two Radon measures with the property that $\mu \ll \lambda$, i.e., that $\mu$ is absolutely continuous with respect to $\lambda$, then (see Lemma 14.6 of [Mat95])

$$
\operatorname{Tan}\left(\mu, x_{0}\right)=\operatorname{Tan}\left(\lambda, x_{0}\right) \quad \text { for } \mu \text {-almost every } x_{0} \in \mathbb{R}^{d},
$$

and in particular if $f \in \mathrm{L}_{\text {loc }}^{1}\left(\mathbb{R}^{d}, \lambda ; \mathbb{R}^{N}\right)$, i.e., $f$ is is $\lambda$-integrable,

$$
\operatorname{Tan}\left(f \lambda, x_{0}\right)=f\left(x_{0}\right) \cdot \operatorname{Tan}\left(\lambda, x_{0}\right) \quad \text { for } \lambda \text {-a.e. } x_{0} \in \mathbb{R}^{d} .
$$

On the other hand, at every $x_{0} \in \operatorname{supp} \mu$ such that

$$
\lim _{r \downarrow 0} \frac{\mu\left(B_{r}\left(x_{0}\right) \backslash E\right)}{\mu\left(B_{r}\left(x_{0}\right)\right)}=0
$$

for some Borel set $E \subset \mathbb{R}^{d}$, it holds that

$$
\operatorname{Tan}\left(\mu, x_{0}\right)=\operatorname{Tan}\left(\mu\left\llcorner E, x_{0}\right) .\right.
$$

A simple consequence of (2.4) is

$$
\operatorname{Tan}\left(|\mu|, x_{0}\right)=\operatorname{Tan}\left(\mathscr{L}^{d}, x_{0}\right) \quad \text { for } \frac{\mathrm{d}|\mu|}{\mathrm{d} \mathscr{L}^{d}} \mathscr{L}^{d} \text {-a.e. } x_{0} \in \mathbb{R}^{d} .
$$

This implies

$$
\operatorname{Tan}\left(\mu, x_{0}\right)=\left\{\alpha \frac{\mathrm{d} \mu}{\mathrm{d} \mathscr{L}^{d}}\left(x_{0}\right) \mathscr{L}^{d}: \alpha \in[0, \infty)\right\} \quad \text { for } \mathscr{L}^{d} \text {-a.e. } x_{0} \in \mathbb{R}^{d} .
$$


We shall refer to such points as regular points of $\mu$. Furthermore, for every regular point $x_{0}$ there exists a sequence $r_{m} \downarrow 0$ and a positive constant $c$ such that

$$
c r_{m}^{-d}\left(T_{\#}^{\left(x_{0}, r_{m}\right)} \mu\right) \stackrel{*}{\rightarrow} \frac{\mathrm{d} \mu}{\mathrm{d} \mathscr{L}^{d}}\left(x_{0}\right) \mathscr{L}^{d} \quad \text { in } \mathscr{M}_{\mathrm{loc}}\left(\mathbb{R}^{d} ; \mathbb{R}^{N}\right) .
$$

2.3. Rigidity results. As discussed in the introduction, for a linear operator $\mathscr{A}:=$ $\sum_{|\alpha| \leq k} A_{\alpha} \partial^{\alpha}$, the wave cone

$$
\Lambda_{\mathscr{A}}:=\bigcup_{|\xi|=1} \operatorname{ker} \mathbb{A}^{k}(\xi) \subset \mathbb{R}^{N}
$$

contains those amplitudes along which is possible to have "one-directional" oscillations or concentrations, or equivalently, it contains the amplitudes along which the system loses its ellipticity.

The main result of [DR16b] asserts that the polar vector of the singular part of an $\mathscr{A}$-free measure $\mu$ necessarily has to lie in $\Lambda_{\mathscr{A}}$ :

Theorem 2.11. Let $\Omega \subset \mathbb{R}^{d}$ be an open set and let $\mu \in \mathscr{M}\left(\Omega ; \mathbb{R}^{N}\right)$ be an $\mathscr{A}$-free Radon measure on $\Omega$ with values in $\mathbb{R}^{N}$, i.e.,

$$
\mathscr{A} \mu=0 \text { in the sense of distributions. }
$$

Then,

$$
\frac{\mathrm{d} \mu}{\mathrm{d}|\mu|}(x) \in \Lambda_{\mathscr{A}} \quad \text { for }\left|\mu^{s}\right| \text {-a.e. } x \in \Omega \text {. }
$$

Remark 2.12. The proof of this result does not require $\mathscr{A}$ to satisfy Murat's constant rank condition (1.2). However, for the present work, this requirement cannot be dispensed with in the following decomposition by Fonseca and Müller [FM99, Lemma 2.14], where it is needed for the Fourier projection arguments.

Lemma 2.13 (projection). Let $\mathscr{A}$ be a homogeneous differential operator satisfying the constant rank property (1.2). Then, for every $1<p<\infty$, there exists a linear projection operator

$$
\mathscr{P}: \mathrm{L}^{p}\left(Q ; \mathbb{R}^{N}\right) \rightarrow \mathrm{L}^{p}\left(Q ; \mathbb{R}^{N}\right)
$$

and a positive constant $c_{p}>0$ such that

$$
\mathscr{A}(\mathscr{P} u)=0, \quad \int_{Q} \mathscr{P} u \mathrm{~d} y=0, \quad\|u-\mathscr{P} u\|_{\mathrm{L}^{p}(Q)} \leq c_{p}\|\mathscr{A} u\|_{\mathrm{W}_{\mathrm{per}}^{-k, p}(Q)},
$$

for every $u \in \mathrm{L}^{p}\left(Q ; \mathbb{R}^{N}\right)$ with $\int_{Q} u \mathrm{~d} y=0$.

Remark 2.14. Here, $\mathrm{W}_{\text {per }}^{k, p}(Q)(1<p<\infty)$ denotes the space of $\mathrm{W}^{k, p}(Q)$-maps, which can be $Q$-periodically extended to a $\mathrm{W}_{\text {loc }}^{k, p}\left(\mathbb{R}^{d}\right)$-map; the space $\mathrm{W}_{\text {per }}^{-k, q}(Q)$ with $1 / p+1 / q=1$ is its dual. Note that the dual norm is equivalent to

$$
\left\|\mathscr{F}^{-1}\left[\frac{\hat{u}(\xi)}{\left(1+|\xi|^{2}\right)^{k / 2}}\right]\right\|_{L^{q}(Q)},
$$

where, for $\xi \in \mathbb{Z}^{d}, \hat{u}(\xi)$ denotes the Fourier coefficients on the torus and $\mathscr{F}^{-1}$ is the inverse Fourier transform. In the case $\int_{Q} u \mathrm{~d} x=0$ (hence $\hat{u}(0)=0$ ) this norm is also equivalent to the norm

$$
\left\|\mathscr{F}^{-1}\left[\frac{\hat{u}(\xi)}{|\xi|^{k}}\right]\right\|_{L^{q}(Q)}
$$


since the Fourier multipliers $\left(1+|\xi|^{2}\right)^{-k / 2}$ and $|\xi|^{-k}$ are comparable (by the Mihlin multiplier theorem) for all $\xi$ with $|\xi| \geq 1$.

Proof. The proof given in [FM99] technically applies only to first-order differential operators. However, the result can be extended to operators of any degree, as long as they are homogeneous. We shortly recall how this is done. By definition,

$$
\operatorname{rank} \mathbb{A}^{k}(\xi)=\operatorname{rank} \mathbb{A}(\xi)=r \text { for all } \xi \in \mathbb{S}^{d-1} .
$$

For each $\xi \in \mathbb{R}^{d}$ we write $\mathbb{P}(\xi): \mathbb{R}^{N} \rightarrow \mathbb{R}^{N}$ to denote the orthogonal projection onto $\operatorname{ker} \mathbb{A}(\xi)$, and by $\mathbb{Q}(\xi)$ we denote the left inverse of $\mathbb{A}(\xi)$.

It follows from the positive homogeneity of $\mathbb{A}$ that $\mathbb{P}: \mathbb{R}^{d} \backslash\{0\} \rightarrow \mathbb{R}^{N} \otimes \mathbb{R}^{N}$ is 0 homogeneous. Moreover, $\operatorname{id}_{\mathbb{R}^{N}}-\mathbb{P}(\xi)=\mathbb{Q}(\lambda \xi) \mathbb{A}(\lambda \xi)=\lambda^{k} \mathbb{Q}(\lambda \xi) \mathbb{A}(\xi)$ and hence $\mathbb{Q}: \mathbb{R}^{d} \backslash\{0\} \rightarrow \mathbb{R}^{N} \otimes \mathbb{R}^{N}$ is homogeneous of degree $-k$. In light of (2.6), both maps are smooth (see Proposition 2.7 in [FM99]).

Since the map $\xi \mapsto \mathbb{P}(\xi)$ is homogeneous of degree 0 and is infinitely differentiable in $\mathbb{S}^{d-1}$, by Proposition 2.13 in [FM99], the map defined on $\mathrm{C}_{\mathrm{per}}^{\infty}\left(Q ; \mathbb{R}^{N}\right)$ by

$$
\mathscr{P} u(w):=\sum_{\xi \in \mathbb{Z}^{d} \backslash\{0\}} \mathbb{P}(\xi) \hat{u}(\xi) e^{2 \pi \mathrm{i} \xi \cdot w},
$$

where $\{\hat{u}(\xi)\}_{\mathbb{Z}^{d}}$ are the Fourier coefficients of $u \in \mathrm{L}^{p}\left(Q ; \mathbb{R}^{N}\right)$, extends to a $(p, p)$ Fourier multiplier $\mathscr{P}$ on $\mathrm{L}^{p}\left(Q ; \mathbb{R}^{N}\right)$ for all $1<p<\infty$.

Since $\mathbb{P}(\xi)$ is a projection, so it is $\mathscr{P}$ :

$$
\begin{aligned}
(\mathscr{P} \circ \mathscr{P}) u & =\sum_{\xi \in \mathbb{Z}^{d} \backslash\{0\}}(\mathbb{P}(\xi) \circ \mathbb{P}(\xi)) \hat{u}(\xi) e^{2 \pi \mathrm{i} \xi \cdot w} \\
& =\sum_{\xi \in \mathbb{Z}^{d} \backslash\{0\}} \mathbb{P}(\xi) \hat{u}(\xi) e^{2 \pi \mathrm{i} \xi \cdot w}=\mathscr{P} u .
\end{aligned}
$$

Moreover,

$$
(\widehat{\mathscr{A}(\mathscr{P} u}))(\xi)=\mathbb{A}(\xi) \widehat{(\mathscr{P} u)}(\xi)=\mathbb{A}(\xi)[\mathbb{P}(\xi) \hat{u}(\xi)]=0
$$

for all $\xi \in \mathbb{Z}^{d} \backslash\{0\}$. Since $\widehat{(\mathscr{P} u)}(0)=0$, we get

$$
\int_{Q} \mathscr{P} u \mathrm{~d} y=0, \quad \text { and } \quad \mathscr{A}(\mathscr{P} u)=0
$$

Finally, let $u \in \mathrm{C}_{\mathrm{per}}^{\infty}\left(Q ; \mathbb{R}^{N}\right)$. We use that $\mathbb{A}$ and $\mathbb{Q}$ are $k$-homogeneous and $(-k)$ homogeneous, respectively, to show that

$$
\begin{aligned}
\hat{u}(\xi)-\widehat{\mathscr{P}} u(\xi) & =\left(\operatorname{id}_{\mathbb{R}^{N}}-\mathbb{P}(\xi)\right) \hat{u}(\xi) \\
& =\mathbb{Q}(\xi) \mathbb{A}(\xi) \hat{u}(\xi) \\
& =\mathbb{Q}\left(\frac{\xi}{|\xi|}\right) \frac{1}{|\xi|^{k}} \mathbb{A}(\xi) \hat{u}(\xi),
\end{aligned}
$$

for all $\xi \in \mathbb{Z}^{d} \backslash\{0\}$. Therefore, the Mihlin multiplier theorem and Remark 2.14 imply that

$$
\|u-\mathscr{P} u\|_{\mathrm{L}^{p}(Q)} \leq c_{p}\|\mathscr{A} u\|_{\mathrm{W}_{\text {per }}^{-k, p}(Q)}
$$

for all $u \in \mathrm{C}_{\mathrm{per}}^{\infty}\left(Q ; \mathbb{R}^{N}\right)$ with $\int_{Q} u \mathrm{~d} y=0$. The general case follows by approximation. 
Lemma 2.13 implies that every $Q$-periodic $u \in \mathrm{L}_{\mathrm{loc}}^{p}\left(\mathbb{R}^{d} ; \mathbb{R}^{N}\right)$ with $1<p<\infty$ and mean value zero can be decomposed as the sum

$$
u=v+w, \quad v=\mathscr{P} u,
$$

where

$$
\mathscr{A} v=0 \quad \text { and } \quad\|w\|_{\mathrm{L}^{p}(Q)} \leq c_{p}\|\mathscr{A} u\|_{\mathrm{W}_{\text {per }}^{-k, p}(Q)} .
$$

A crucial issue in lower semicontinuity problems is the understanding of oscillation and concentration effects in weakly (weakly*) convergent sequences. In our setting, we are interested in sequences of asymptotically $\mathscr{A}$-free measures generating what we naturally term $\mathscr{A}$-free Young measures. The study of general $\mathscr{A}$-free Young measures can be reduced to understanding oscillations in the class of periodic $\mathscr{A}$ free fields. This is expressed in the next lemma, which is a variant of Proposition 3.1 in [FLM04] for higher-order operators (see also Lemma 2.20 in [BCMS13]).

Lemma 2.15. Let $\mathscr{A}$ be an homogeneous linear partial differential operator satisfying the constant rank property $(1.2)$. Let $\left(u_{j}\right),\left(v_{j}\right) \subset \mathrm{L}^{1}\left(Q ; \mathbb{R}^{N}\right)$ be sequences such that

$$
u_{j}-v_{j} \stackrel{*}{\rightarrow} 0 \quad \text { in } \mathscr{M}\left(Q ; \mathbb{R}^{N}\right) \quad \text { and } \quad\left|u_{j}\right|+\left|v_{j}\right| \stackrel{*}{\rightarrow} \Lambda \quad \text { in } \mathscr{M}^{+}(\bar{Q})
$$

with $\Lambda(\partial Q)=0$ and

$$
\mathscr{A}\left(u_{j}-v_{j}\right) \rightarrow 0 \quad \text { in } \mathrm{W}^{-k, q}\left(Q ; \mathbb{R}^{n}\right) \quad \text { for some } 1<q<d /(d-1) .
$$

Assume that the sequence $\left(u_{j}\right)$ generates the Young measure $v \in \mathbf{Y}\left(Q ; \mathbb{R}^{N}\right)$. Then, there exists another sequence $\left(z_{j}\right) \subset \mathrm{C}_{\text {per }}^{\infty}\left(Q ; \mathbb{R}^{N}\right)$ such that

$$
\mathscr{A} z_{j}=0, \quad \int_{Q} z_{j}=0, \quad z_{j} \stackrel{*}{\rightarrow} 0 \quad \text { in } \mathscr{M}\left(Q ; \mathbb{R}^{N}\right),
$$

and (up to taking a subsequence of the $v_{j}$ 's) the sequence $\left(v_{j}+z_{j}\right)$ also generates the Young measure $v$, i.e.,

$$
\left(v_{j}+z_{j}\right) \stackrel{\mathbf{Y}}{\rightarrow} v \quad \text { in } \mathbf{Y}\left(Q ; \mathbb{R}^{N}\right) .
$$

Moreover, for every $f: \mathbb{R}^{N} \rightarrow \mathbb{R}$ Lipschitz it holds that

$$
\liminf _{j \rightarrow \infty} \int_{Q} f\left(u_{j}\right) \mathrm{d} x \geq \liminf _{j \rightarrow \infty} \int_{Q} f\left(v_{j}+z_{j}\right) \mathrm{d} x .
$$

Proof. Consider a family of cut-off functions $\psi_{m} \in \mathrm{C}_{c}^{\infty}(Q ;[0,1])$ with $\psi_{m} \equiv 1$ in the set $\{y \in Q: \operatorname{dist}(y, \partial Q)>1 / m\}$ and define

$$
w_{j}^{m}:=\left(u_{j}-v_{j}\right) \psi_{m} \in \mathrm{L}^{1}\left(Q ; \mathbb{R}^{N}\right) .
$$

Since $\psi_{m} \in \mathrm{C}_{c}^{\infty}(Q)$, it also holds that

$$
w_{j}^{m} \stackrel{*}{\rightarrow} 0 \quad \text { in } \mathscr{M}\left(Q ; \mathbb{R}^{N}\right) \quad \text { as } j \rightarrow \infty, \quad \text { for every } m \in \mathbb{N} .
$$

Furthermore,

$$
\mathscr{A} w_{j}^{m}=\mathscr{A}\left(u_{j}-v_{j}\right) \psi_{m}+\sum_{\substack{|\alpha|=k, 1 \leq|\beta| \leq k}} c_{\alpha \beta} A_{\alpha} \partial^{\alpha-\beta}\left(u_{j}-v_{j}\right) \partial^{\beta} \psi_{m}
$$

where $c_{\alpha \beta} \in \mathbb{N}$. The convergence $u_{j}-v_{j} \stackrel{*}{\rightarrow} 0$ and the compact embedding $\mathscr{M}\left(Q ; \mathbb{R}^{N}\right) \stackrel{c}{\hookrightarrow}$ $\mathrm{W}^{-1, q}\left(Q ; \mathbb{R}^{N}\right)$ entail, via (2.8), the strong convergence

$$
\mathscr{A} w_{j}^{m} \rightarrow 0 \quad \text { in } \mathrm{W}^{-k, q}\left(Q ; \mathbb{R}^{n}\right) \quad \text { as } j \rightarrow \infty .
$$


Let, for $\varepsilon>0, \rho_{\varepsilon}(x):=\rho(x / \varepsilon)$ where $\rho \in \mathrm{C}_{c}^{\infty}\left(B_{1}\right)$ is an even mollifier. For every $m \in \mathbb{N}$, let $(\varepsilon(j, m))_{j}$ be a sequence with $\varepsilon(j, m) \downarrow 0$ as $j \rightarrow \infty$ such that for $\hat{w}_{j}^{m}:=$ $w_{j}^{m} * \rho_{\varepsilon(j, m)}$ it holds that

$$
\left\|w_{j}^{m}-\hat{w}_{j}^{m}\right\|_{L^{1}(Q)} \leq \frac{1}{j} .
$$

Fix $\varphi \in \mathrm{W}^{k, q}\left(Q ; \mathbb{R}^{n}\right) \cap \mathrm{C}_{c}\left(Q ; \mathbb{R}^{n}\right)$ and fix $m \in \mathbb{N}$. Then, for $j \in \mathbb{N}$ sufficiently large, it holds that

$$
\begin{aligned}
\left|\left\langle\mathscr{A} \hat{w}_{j}^{m}, \varphi\right\rangle\right| & =\left|\left\langle\mathscr{A} w_{j}^{m}, \varphi * \rho_{\varepsilon(j, m)}\right\rangle\right| \\
& \leq\left\|\mathscr{A} w_{j}^{m}\right\|_{\mathrm{W}^{-k, q}(Q)}\left\|\varphi * \rho_{\varepsilon(j, m)}\right\|_{\mathrm{W}^{k, q}(Q)} \\
& \leq\left\|\mathscr{A} w_{j}^{m}\right\|_{\mathrm{W}^{-k, q}(Q)}\|\varphi\|_{\mathrm{W}^{k, q}(Q)} .
\end{aligned}
$$

The case when $\varphi$ belongs to $\mathrm{W}_{0}^{k, q}\left(Q ; \mathbb{R}^{n}\right)$ follows by approximation. Hence, from (2.9) we obtain that

$$
\left\|\mathscr{A} \hat{w}_{j}^{m}\right\|_{\mathrm{W}^{-k, q}(Q)} \rightarrow 0 \quad \text { as } j \rightarrow \infty, \quad \text { for every } m \in \mathbb{N} .
$$

The second step consists of applying the projection of Lemma 2.13 to the mollified functions $\hat{w}_{j}^{m}$. Define $\tilde{w}_{j}^{m}:=\hat{w}_{j}^{m}-\int_{Q} \hat{w}_{j}^{m} \mathrm{~d} x$ (by a slight abuse of notation, we also denote by $\tilde{w}_{j}^{m}$ its $Q$-periodic extension to $\mathbb{R}^{d}$ ) and $z_{j}^{m}:=\mathscr{P} \tilde{w}_{j}^{m}$. Note that since $\tilde{w}_{j}^{m} \in \mathrm{C}^{\infty}(Q)$ the same holds for $z_{j}^{m}$ since the projection operators commutes with the Fourier multiplier $|\xi|^{s}$ for all $s \in \mathbb{R}$. It follows from Lemma 2.13 that

$$
\begin{aligned}
\lim _{j \rightarrow \infty}\left\|\hat{w}_{j}^{m}-z_{j}^{m}\right\|_{\mathrm{L}^{1}(Q)} & \leq \lim _{j \rightarrow \infty}\left\|\tilde{w}_{j}^{m}-z_{j}^{m}\right\|_{\mathrm{L}^{q}(Q)}+\lim _{j \rightarrow \infty}\left|\int_{Q} \hat{w}_{j}^{m} \mathrm{~d} y\right| \\
& \leq c_{q} \cdot \lim _{j \rightarrow \infty}\left\|\mathscr{A} \hat{w}_{j}^{m}\right\|_{\mathrm{W}_{\mathrm{per}}^{-k, q}(Q)}+\lim _{j \rightarrow \infty}\left|\int_{Q} w_{j}^{m} \mathrm{~d} y\right| \\
& =0,
\end{aligned}
$$

where in the first inequality we have exploited Jensen's inequality, and for the last inequality we have used the equality of the norms

$$
\|u\|_{\mathrm{W}_{\mathrm{per}}^{-k, p}(Q)}=\|u\|_{\mathrm{W}^{-k, p}(Q)},
$$

which holds for functions $u \in \mathrm{C}_{\text {per }}^{\infty}(Q)$ with $u=0$ on $\partial Q$ and all $1<p<\infty$, together with (2.10).

Let now $g: \mathbb{R}^{N} \rightarrow \mathbb{R}$ be Lipschitz and let $\varphi \in \mathrm{C}(\bar{Q})$ with $\varphi \geq 0$. Then,

$$
\begin{aligned}
\int_{Q} \varphi g\left(u_{j}\right) \mathrm{d} y= & \int_{Q} \varphi g\left(u_{j}-v_{j}+v_{j}\right) \mathrm{d} y \\
\geq & \int_{Q} \varphi g\left(\hat{w}_{j}^{m}+v_{j}\right) \mathrm{d} y-\|\varphi\|_{\infty} \cdot \operatorname{Lip}(g) \cdot \int_{Q}\left|1-\psi_{m}\right|\left(\left|u_{j}\right|+\left|v_{j}\right|\right) \mathrm{d} y \\
& \quad-\|\varphi\|_{\infty} \cdot \operatorname{Lip}(g) \cdot\left\|w_{j}^{m}-\hat{w}_{j}^{m}\right\|_{\mathrm{L}^{1}(Q)} \\
\geq & \int_{Q} \varphi g\left(z_{j}^{m}+v_{j}\right) \mathrm{d} y-\|\varphi\|_{\infty} \cdot \operatorname{Lip}(g) \cdot\left(\int_{Q}\left|1-\psi_{m}\right|\left(\left|u_{j}\right|+\left|v_{j}\right|\right) \mathrm{d} y\right. \\
& \left.\quad+\left\|w_{j}^{m}-\hat{w}_{j}^{m}\right\|_{\mathrm{L}^{1}(Q)}+\left\|\hat{w}_{j}^{m}-z_{j}^{m}\right\|_{\mathrm{L}^{1}(Q)}\right) .
\end{aligned}
$$


Similarly,

$$
\begin{gathered}
\int_{Q} \varphi g\left(u_{j}\right) \mathrm{d} y \leq \int_{Q} \varphi \\
g\left(z_{j}^{m}+v_{j}\right) \mathrm{d} y+\|\varphi\|_{\infty} \cdot \operatorname{Lip}(g) \cdot\left(\int_{Q}\left|1-\psi_{m}\right|\left(\left|u_{j}\right|+\left|v_{j}\right|\right) \mathrm{d} y\right. \\
\left.+\left\|w_{j}^{m}-\hat{w}_{j}^{m}\right\|_{\mathrm{L}^{1}(Q)}+\left\|\hat{w}_{j}^{m}-z_{j}^{m}\right\|_{\mathrm{L}^{1}(Q)}\right)
\end{gathered}
$$

Let $\left\{\varphi_{h} \otimes g_{h}\right\}_{h=1}^{\infty}$ be the family of integrands appearing in Lemma 2.4 and let $v$ be the Young measure generated by $\left(u_{j}\right)$. We have that

$$
\lim _{j \rightarrow \infty} \int_{Q} \varphi_{h} g_{h}\left(u_{j}\right)=\left\langle\left\langle\varphi_{m} \otimes g_{m}, v\right\rangle \quad \text { for all } h=1,2, \ldots\right.
$$

and thus using (2.12) and (2.13) above we infer that

$$
\begin{aligned}
\limsup _{m \rightarrow \infty} \limsup _{j \rightarrow \infty} \int_{Q} \varphi_{h} g_{h}\left(z_{j}^{m}+v_{j}\right) \mathrm{d} y & \leq \lim _{j \rightarrow \infty} \int_{Q} \varphi_{h} g_{h}\left(u_{j}\right) \mathrm{d} y \\
& \leq \liminf _{m \rightarrow \infty} \liminf _{j \rightarrow \infty} \int_{Q} \varphi_{h} g_{h}\left(z_{j}^{m}+v_{j}\right) \mathrm{d} y .
\end{aligned}
$$

for all $h \in \mathbb{N}$ where we have also exploited that $\Lambda(\partial Q)=0$. By a diagonalization argument on $z_{j}^{m}$ we may find a sequence $\left(z_{j}\right) \subset \mathrm{C}_{\mathrm{per}}^{\infty}\left(Q ; \mathbb{R}^{N}\right) \cap \operatorname{ker} \mathscr{A}$ such that

$$
\int_{Q} z_{j} \mathrm{~d} y=0 \quad \text { for all } j \in \mathbb{N}, \quad z_{j} \stackrel{*}{\rightarrow} 0 \quad \text { in } \mathscr{M}\left(Q ; \mathbb{R}^{N}\right),
$$

and, for all $h \in \mathbb{N}$,

$$
\lim _{j \rightarrow \infty} \int_{Q} \varphi_{h} g_{h}\left(u_{j}\right) \mathrm{d} y=\lim _{j \rightarrow \infty} \int_{Q} \varphi_{h} g_{h}\left(z_{j}+v_{j}\right) \mathrm{d} y .
$$

Since $\left(z_{j}+v_{j}\right)$ is uniformly bounded in $\mathrm{L}^{1}\left(Q ; \mathbb{R}^{N}\right)$, by Lemma 2.5 we may find a subsequence $\left(z_{j(i)}+v_{j(i)}\right) \stackrel{\mathbf{Y}}{\rightarrow} \tilde{v} \in \mathbf{Y}\left(Q ; \mathbb{R}^{N}\right)$. In particular,

$$
\left\langle\varphi_{h} \otimes g_{h}, \tilde{v}\right\rangle=\left\langle\left\langle\varphi_{h} \otimes g_{h}, v\right\rangle,\right.
$$

for all $h$ and thus $\tilde{v}=v$ by Lemma 2.4. Inequality (2.7) now follows by taking the limit inferior in (2.12) with $g=f$ and $\varphi \equiv 1$.

2.4. Scaling properties of $\mathscr{A}$-free measures. If $\mathscr{A}$ is a homogeneous operator, then

$$
\mathscr{A}\left[T_{\#}^{\left(x_{0}, r\right)} \mu\right]=0 \quad \text { on }\left(x_{0}-\Omega\right) / r
$$

for all $\mathscr{A}$-free measures $\mu \in \mathscr{M}\left(\Omega ; \mathbb{R}^{N}\right)$. In general, the re-scaled measure $T_{\#}^{\left(x_{0}, r\right)} \mu$ is a $\left(T_{*}^{r} \mathscr{A}\right)$-free measure in $\left(x_{0}-\Omega\right) / r$, where $T_{*}^{r} \mathscr{A}$ is the operator defined by

$$
T_{*}^{r} \mathscr{A}:=\sum_{h=0}^{k} r^{k-h} \mathscr{A}^{h}
$$

with $k$ the degree of the operator $\mathscr{A}$ and

$$
\mathscr{A}^{h}:=\sum_{|\alpha|=h} A_{\alpha} \partial^{\alpha}, \quad \text { for } h=0, \ldots, k .
$$

Notice that, with this convention, $\left(T_{*}^{r} \mathscr{A}\right)^{k}=\mathscr{A}^{k}$.

In the sequel it will be often convenient to work with weak* convergent sequences whose elements are $\left(T_{*}^{r} \mathscr{A}\right)$-free measures. The following two results will be useful. 
Proposition 2.16. Let $r_{m} \downarrow 0$ be a sequence of positive numbers and let $\left(\mu_{m}\right)$ be a sequence of $\mathscr{A}$-free measures in $\mathscr{M}\left(\Omega ; \mathbb{R}^{N}\right)$ with the following property: there are positive constants $c_{m}$ such that

$$
\gamma_{m}:=c_{m} T_{\#}^{\left(x_{0}, r_{m}\right)} \mu_{m} \stackrel{*}{\rightarrow} \gamma \quad \text { in } \mathscr{M}_{\mathrm{loc}}\left(\mathbb{R}^{d} ; \mathbb{R}^{N}\right) .
$$

Then,

$$
\mathscr{A}^{k}\left(c_{m} T_{\#}^{\left(x_{0}, r_{m}\right)} \mu_{m}\right) \rightarrow 0 \quad \text { in } \mathrm{W}_{\mathrm{loc}}^{-k, q}\left(\mathbb{R}^{d} ; \mathbb{R}^{n}\right) \quad \text { for all } 1<q<d /(d-1) .
$$

Proof. Fix $r>0$. Then,

$$
\mathscr{A}^{k}\left(T_{\#}^{\left(x_{0}, r\right)} \mu_{m}\right)=-\sum_{h=0}^{k-1} \mathscr{A}^{h}\left(r^{k-h} T_{\#}^{\left(x_{0}, r\right)} \mu_{m}\right) .
$$

Since

$$
r_{m}^{k-h} c_{m} T_{\#}^{\left(x_{0}, r_{m}\right)} \mu_{m} \stackrel{*}{\rightarrow} 0 \quad \text { in } \mathscr{M}_{\mathrm{loc}}\left(\mathbb{R}^{d} ; \mathbb{R}^{N}\right), \quad \text { for every } h=0, \ldots, k-1,
$$

the compact embedding $\mathscr{M}_{\mathrm{loc}}\left(\mathbb{R}^{d} ; \mathbb{R}^{N}\right) \stackrel{c}{\hookrightarrow} \mathrm{W}_{\text {loc }}^{-1, q}\left(\mathbb{R}^{d} ; \mathbb{R}^{N}\right)$ entails the strong convergence

$$
r_{m}^{k-h} c_{m} T_{\#}^{\left(x_{0}, r_{m}\right)} \mu_{m} \rightarrow 0 \quad \text { in } \mathrm{W}_{\mathrm{loc}}^{-1, q}\left(\mathbb{R}^{d} ; \mathbb{R}^{N}\right) \quad \text { for every } h=0, \ldots, k-1 .
$$

Hence,

$$
\mathscr{A}^{h}\left(r_{m}^{k-h} c_{m} T_{\#}^{\left(x_{0}, r\right)} \mu_{m}\right) \rightarrow 0 \quad \text { in } \mathrm{W}_{\mathrm{loc}}^{-k, q}\left(\mathbb{R}^{d} ; \mathbb{R}^{n}\right)
$$

for every $h=0, \ldots, k-1$. The assertion then follows from (2.16) and (2.17).

2.5. Fourier coefficients of $\mathscr{A}^{k}$-free sequences. We shall denote the subspace generated by the wave cone $\Lambda_{\mathscr{A}}$ by

$$
V_{\mathscr{A}}:=\operatorname{span} \Lambda_{\mathscr{A}} \subset \mathbb{R}^{N} .
$$

Using Fourier series it is relatively easy to understand the rigidity of $\mathscr{A}^{k}$-free periodic fields. To fix ideas, let $u$ be a $Q$-periodic field in $\mathrm{L}_{\text {loc }}^{2}\left(\mathbb{R}^{d} ; \mathbb{R}^{N}\right) \cap \operatorname{ker} \mathscr{A}^{k}$ with mean value zero (or equivalently $\hat{u}(0)=0$ ). Applying the Fourier transform to $\mathscr{A}^{k} u=0$, we find that

$$
0=\mathscr{F}\left(\mathscr{A}^{k} u\right)(\xi)=\mathbb{A}^{k}(\xi) \hat{u}(\xi) \quad \text { for all } \xi \in \mathbb{Z}^{d} .
$$

Hence, $\hat{u}(\xi) \in \operatorname{ker}_{\mathbb{C}} \mathbb{A}^{k}(\xi)$ for every $\xi \in \mathbb{Z}^{d}$ (here, $\mathbb{A}^{k}(\xi)$ is understood as a complexvalued tensor). In particular,

$$
\left\{\hat{u}(\xi): \xi \in \mathbb{Z}^{d}\right\} \subset \mathbb{C} \Lambda_{\mathscr{A}} .
$$

Since $u$ is a real vector-valued function, it immediately follows that

$$
u \in \mathrm{L}_{\text {loc }}^{2}\left(\mathbb{R}^{d} ; V_{\mathscr{A}}\right) .
$$

Using a density argument one can show that, up to a constant term, also $Q$-periodic functions in $\mathrm{L}_{\text {loc }}^{1}\left(Q ; \mathbb{R}^{N}\right) \cap \operatorname{ker} \mathscr{A}^{k}$ take values only in $V_{\mathscr{A}}$. The relevance of this observation will be used later in conjunction with Lemma 2.15 in Lemma 3.2. 
2.6. $\mathscr{A}$-quasiconvexity. We state some well-known and some more recent results regarding the properties of $\mathscr{A}$-quasiconvex integrands. This notion was first introduced by Morrey [Mor66] in the case of curl-free vector fields, where it is known as quasiconvexity, and later extended by Dacorogna [Dac82] and Fonseca-Müller [FM99] to general linear PDE-constraints.

A Borel function $h: \mathbb{R}^{N} \rightarrow \mathbb{R}$ is called $\mathscr{A}$-quasiconvex if

$$
h(A) \leq \int_{Q} h(A+w(y)) \mathrm{d} y
$$

for all $A \in \mathbb{R}^{N}$ and all $Q$-periodic $w \in \mathrm{C}^{\infty}\left(\mathbb{R}^{d} ; \mathbb{R}^{N}\right)$ such that

$$
\mathscr{A} w=0 \quad \text { and } \quad \int_{Q} w \mathrm{~d} x=0 .
$$

For functions $h$ that are not $\mathscr{A}$-quasiconvex one may define the largest $\mathscr{A}$-quasiconvex function below $h$.

Definition 2.17 ( $\mathscr{A}$-quasiconvex envelope). Given a Borel function $h: \mathbb{R}^{N} \rightarrow \mathbb{R}$ we define the $\mathscr{A}$-quasiconvex envelope of $h$ at $A \in \mathbb{R}^{N}$ as

$$
\left(Q_{\mathscr{A}} h\right)(A):=\inf \left\{\int_{Q} h(A+w(y)) \mathrm{d} y: w \in \mathrm{C}_{\text {per }}^{\infty}\left(Q ; \mathbb{R}^{N}\right) \cap \operatorname{ker} \mathscr{A}, \int_{Q} w \mathrm{~d} y=0\right\} .
$$

For a map $f: \Omega \times \mathbb{R}^{N} \rightarrow \mathbb{R}$ we write $Q_{\mathscr{A}} f(x, A)$ for $\left(Q_{\mathscr{A}} f(x, \cdot)\right)(A)$ by a slight abuse of notation.

We recall from [FM99] that the $\mathscr{A}$-quasiconvex envelope of an upper semicontinuous function is $\mathscr{A}$-quasiconvex and that it is actually the largest $\mathscr{A}$-quasiconvex function below $h$.

Lemma 2.18. If $h: \mathbb{R}^{N} \rightarrow[0, \infty)$ is upper semicontinuous, then $Q_{\mathscr{A}}$ h is upper semicontinuous and $\mathscr{A}$-quasiconvex. Furthermore, $Q_{\mathscr{A}}$ h is the largest $\mathscr{A}$-quasiconvex function below $h$.

2.7. $\mathscr{D}$-convexity. Let $\mathscr{D}$ be a balanced cone of directions in $\mathbb{R}^{N}$, i.e., we assume that $t A \in \mathscr{D}$ for all $A \in \mathscr{D}$ and every $t \in \mathbb{R}$. A real-valued function $h: \mathbb{R}^{N} \rightarrow \mathbb{R}$ is said to be $\mathscr{D}$-convex provided its restrictions to all line segments in $\mathbb{R}^{N}$ with directions in $\mathscr{D}$ are convex. Here, $\mathscr{D}$ will always be the wave cone $\Lambda_{\mathscr{A}}$ for the linear PDE operator $\mathscr{A}$.

Lemma 2.19. Let $h: \mathbb{R}^{N} \rightarrow[0, \infty)$ be an integrand with linear growth at infinity. Further, suppose that $h$ is $\mathscr{A}^{k}$-quasiconvex. Then, $h$ is $\Lambda_{\mathscr{A}}$-convex.

Proof. Let $\xi \in \mathbb{S}^{d-1}$ and let $A_{1}, A_{2} \in \mathbb{R}^{d}$ with $P:=A_{1}-A_{2} \in \operatorname{ker} \mathbb{A}^{k}(\xi)$. We claim that

$$
h\left(\theta A_{1}+(1-\theta) A_{2}\right) \leq \theta h\left(A_{1}\right)+(1-\theta) h\left(A_{2}\right), \quad \text { for all } \theta \in(0,1) .
$$

Fix such a $\theta$ and consider the one-dimensional 1-periodic function

$$
\chi(s):=(1-\theta) \mathbb{1}_{[0, \theta)}(s)-\theta \mathbb{1}_{[\theta, 1)}(s), \quad s \in[0,1),
$$

which has zero mean value. Fix $\varepsilon \in \min \{\theta / 2,(1-\theta) / 2\}$ so that the mollified function $\chi_{\varepsilon}:=\chi * \rho_{\varepsilon}$ has the following properties:

$$
\left|\left\{s: \chi_{\varepsilon}=1-\theta\right\}\right| \geq \theta-2 \varepsilon, \quad\left|\left\{s: \chi_{\varepsilon}=-\theta\right\}\right| \geq(1-\theta)-2 \varepsilon .
$$


Define the sequence of $Q$-periodic functions

$$
u_{\varepsilon}:=P \chi_{\varepsilon}(y \cdot \xi) .
$$

By construction, this is a $\mathrm{C}_{\text {per }}^{\infty}\left(Q ; \mathbb{R}^{N}\right)$ function, it has zero mean value in $Q$, and since $P \in \operatorname{ker} \mathbb{A}^{k}(\xi)$, it is easy to check that

$$
\mathscr{A}^{k} u_{\varepsilon}=(2 \pi \mathrm{i})^{-k} \frac{\mathrm{d}^{k} \chi_{\varepsilon}}{\mathrm{d} s^{k}}(y \cdot \xi) \mathbb{A}^{k}(\xi) P=0 \quad \text { in the sense of distributions. }
$$

Hence, by the definition of $\mathscr{A}^{k}$-quasiconvexity and our choice of $\varepsilon$, we have

$$
\begin{aligned}
h\left(\theta A_{1}+(1-\theta) A_{2}\right) \leq & \int_{Q} h\left(\theta A_{1}+(1-\theta) A_{2}+u_{\varepsilon}\right) \mathrm{d} y \\
\leq & (\theta-2 \varepsilon) h\left(A_{1}\right)+((1-\theta)-2 \varepsilon) h\left(A_{2}\right) \\
& +M\left(1+\left|A_{1}\right|+\left|A_{2}\right|+|P|\right) 4 \varepsilon
\end{aligned}
$$

Letting $\varepsilon \downarrow 0$ in the previous inequality yields the claim.

The following is an immediate consequence of Lemmas 2.18 and 2.19.

Corollary 2.20. If $h: \mathbb{R}^{N} \rightarrow[0, \infty)$ is upper semicontinuous, then $\left(Q_{\mathscr{A}^{k}} h\right)^{\#}$ is an $\mathscr{A}^{k}$-quasiconvex and $\Lambda_{\mathscr{A}}$-convex function.

To continue our discussion we define the notion of convexity at a point. Let $h$ : $\mathbb{R}^{N} \rightarrow \mathbb{R}$ be a Borel function. We recall that Jensen's definition of convexity states that $h$ is convex if and only if

$$
f\left(\int_{\mathbb{R}^{N}} A \mathrm{~d} v(A)\right) \leq \int_{\mathbb{R}^{N}} h(A) \mathrm{d} v(A)
$$

for all probability measures $v \in \mathscr{M}^{1}\left(\mathbb{R}^{N}\right)$.

A Borel function $h: \mathbb{R}^{N} \rightarrow \mathbb{R}$ is said to be convex at a point $A_{0} \in \mathbb{R}^{N}$ if (2.19) holds for for all probability measures $v$ with barycenter $A_{0}$, that is, every $v \in \mathscr{M}^{1}\left(\mathbb{R}^{N}\right)$ with $\int_{\mathbb{R}^{N}} A \mathrm{~d} v=A_{0}$.

Returning to the convexity properties of $\mathscr{A}^{k}$-quasiconvex functions, it was recently shown by Kirchheim and Kristensen [KK11, KK16] that $\mathscr{A}^{k}$-quasiconvex and positively 1-homogeneous integrands are actually convex at points of $\Lambda_{\mathscr{A}}$ as long as

$$
\operatorname{span} \Lambda_{\mathscr{A}}=\mathbb{R}^{N} .
$$

In fact, their result is valid in the more general framework of $\mathscr{D}$-convexity:

Theorem 2.21 (Theorem 1.1 of [KK16]). Let $\mathscr{D}$ be a balanced cone of directions in $\mathbb{R}^{N}$ and assume that $\mathscr{D}$ spans $\mathbb{R}^{N}$. If $h: \mathbb{R}^{N} \rightarrow \mathbb{R}$ is $\mathscr{D}$-convex and positively 1 homogeneous, then $h$ is convex at each point of $\mathscr{D}$.

Condition (2.20) holds in several applications, for example in the space of gradients $(\mathscr{A}=$ curl $)$ or the space of divergence-free fields $(\mathscr{A}=$ div $)$. However, it does not necessarily hold in our framework as it is evidenced by the operator

$$
\mathscr{A}:=A_{0} \Delta=\sum_{i=1}^{d} A_{0} \partial_{i i}
$$

where $A_{0} \in \mathbb{R}^{n} \otimes \mathbb{R}^{N}$ with $\operatorname{ker} A_{0} \neq \mathbb{R}^{N}$.

Nevertheless, for our purposes it will be sufficient to use the convexity of $\left.f^{\#}\right|_{V_{\mathscr{A}}}(x, \bullet)$ in $\Lambda_{\mathscr{A}}$, which is a direct consequence of Theorem 2.21. 
Remark 2.22 (automatic convexity). Summing up, in the following we will often make use of the implications from Lemma 2.18, Corollary 2.20 and Theorem 2.21: If $f: \Omega \times \mathbb{R}^{N} \rightarrow \mathbb{R}$ is an integrand with linear growth at infinity, then

$$
\begin{gathered}
f(x, \cdot) \text { is } \mathscr{A}^{k} \text {-quasiconvex } \Longrightarrow\left\{\begin{array}{c}
f(x, \cdot) \text { is } \Lambda_{\mathscr{A}} \text {-convex in } \mathbb{R}^{N} \text { and } \\
\left.f^{\#}\right|_{V_{\mathscr{A}}}(x, \cdot) \text { is convex at points in } \Lambda_{\mathscr{A}}
\end{array}\right. \\
f \text { upper semicontinuous } \Longrightarrow\left\{\begin{array}{c}
Q_{\mathscr{A}^{k}} f(x, \cdot) \text { is } \Lambda_{\mathscr{A}} \text {-convex in } \mathbb{R}^{N} \text { and } \\
\left.\left(Q_{\mathscr{A}^{k}} f\right)^{\#}\right|_{V_{\mathscr{A}}}(x, \cdot) \text { is convex at points in } \Lambda_{\mathscr{A}}
\end{array} .\right.
\end{gathered}
$$

2.8. Localization principles for Young measures. We state two general localization principles for Young measures, one at regular points and another one at singular points. These are $\mathscr{A}$-free versions of the localization principles developed for gradient Young measures and BD-Young measures in [Rin11, Rin12].

Definition 2.23 ( $\mathscr{A}$-free Young measure). We say that a Young measure $v \in \mathbf{Y}\left(\Omega ; \mathbb{R}^{N}\right)$ is an $\mathscr{A}$-free Young measure in $\Omega$, in symbols $v \in \mathbf{Y}_{\mathscr{A}}\left(\Omega ; \mathbb{R}^{N}\right)$, if and only if there exists a sequence $\left(\mu_{j}\right) \subset \mathscr{M}\left(\Omega ; \mathbb{R}^{N}\right)$ with $\mathscr{A} \mu_{j} \rightarrow 0$ in $\mathrm{W}^{-k, q}$ for some $1<q<$ $d /(d-1)$, and such that $\mu_{j} \stackrel{\mathbf{Y}}{\rightarrow} v$ in $\mathbf{Y}\left(\Omega ; \mathbb{R}^{N}\right)$.

Proposition 2.24. Let $v \in \mathbf{Y}_{\mathscr{A}}\left(\Omega ; \mathbb{R}^{N}\right)$ be an $\mathscr{A}$-free Young measure. Then for $\mathscr{L}^{d}$ a.e. $x_{0} \in \Omega$ there exists a regular tangent $\mathscr{A}^{k}$-free Young measure $\sigma \in \mathbf{Y}_{\mathscr{A} k}\left(Q ; \mathbb{R}^{N}\right)$ to $v$ at $x_{0}$, that is, $\sigma$ is generated by a sequence of asymptotically $\mathscr{A}^{k}$-free measures and

$$
\begin{aligned}
{[\sigma] } & \in \operatorname{Tan}_{Q}\left([v], x_{0}\right), & & \sigma_{y}=v_{x_{0}} \text { a.e. }, \\
\lambda_{\sigma}=\frac{\mathrm{d} \lambda_{v}}{\mathrm{~d} \mathscr{L}^{d}}\left(x_{0}\right) \mathscr{L}^{d} & \in \operatorname{Tan}_{Q}\left(\lambda_{v}, x_{0}\right), & \sigma_{y}^{\infty} & =v_{x_{0}}^{\infty} \lambda_{\sigma} \text {-a.e. }
\end{aligned}
$$

Moreover, there exists a sequence $\left(w_{j}\right) \subset \mathrm{C}_{\mathrm{per}}^{\infty}\left(Q ; \mathbb{R}^{N}\right) \cap \operatorname{ker} \mathscr{A}^{k}$ such that $w_{j} \mathscr{L}^{d} \stackrel{\mathrm{Y}}{\rightarrow} \sigma$ in $\mathbf{Y}\left(Q ; \mathbb{R}^{N}\right)$.

Proposition 2.25. Let $v \in \mathbf{Y}_{\mathscr{A}}\left(\Omega ; \mathbb{R}^{N}\right)$ be an $\mathscr{A}$-free Young measure. Then there exists a set $S \subset \Omega$ with $\lambda_{v}^{s}(\Omega \backslash S)=0$ such that for all $x_{0} \in S$ there exists a non-zero singular tangent $\mathscr{A}^{k}$-free Young measure $\sigma \in \mathbf{Y}_{\mathscr{A}^{k}}\left(Q ; \mathbb{R}^{N}\right)$ to $v$ at $x_{0}$, that is, $\sigma$ is generated by a sequence of asymptotically $\mathscr{A}^{k}$-free measures and

$$
\begin{gathered}
{[\sigma] \in \operatorname{Tan}_{Q}\left([v], x_{0}\right), \quad \sigma_{y}=\delta_{0} \text { a.e. }} \\
\lambda_{\sigma} \in \operatorname{Tan}_{Q}\left(\lambda_{v}^{s}, x_{0}\right), \quad \lambda_{\sigma}(Q)=1, \quad \lambda_{\sigma}(\partial Q)=0, \quad \sigma_{y}^{\infty}=v_{x_{0}}^{\infty} \lambda_{\sigma^{-}} \text {a.e. }
\end{gathered}
$$

The proofs for the first part of the statements above are by now standard (see, for instance, [Rin12]). The existence of an $\mathscr{A}^{k}$-free generating sequence in Proposition 2.24 is obtained by Lemma 2.15. For the sake of readability, the proofs are postponed to the appendix.

\section{JENSEN'S INEQUALITIES}

In this section we establish generalized Jensen inequalities, which can be understood as a local manifestation of lower semicontinuity. The proof of Theorem 1.2, 
under Assumption (i), which reads

$$
f^{\infty}(x, A):=\lim _{t \rightarrow \infty} \frac{f(x, t A)}{t} \quad \text { exists for all }(x, A) \in \Omega \times \mathbb{R}^{N},
$$

will easily follow from Propositions 3.1 and 3.3.

On the other hand, to prove the Theorem 1.2 under the weaker Assumption (ii),

$$
f^{\infty}(x, A):=\lim _{t \rightarrow \infty} \frac{f(x, t A)}{t} \quad \text { exists for all } \quad(x, A) \in \Omega \times \operatorname{span} \Lambda_{\mathscr{A}},
$$

requires to perform a direct blow-up argument for what concerns the regular part of $\mu$ and only Proposition 3.3 is used in the proof.

3.1. Jensen's inequality at regular points. We first consider regular points.

Proposition 3.1. Let $v \in \mathbf{Y}_{\mathscr{A}}\left(\Omega ; \mathbb{R}^{N}\right)$ be an $\mathscr{A}$-free Young measure. Then, for $\mathscr{L}^{d}$ almost every $x_{0} \in \Omega$ it holds that

$$
h\left(\left\langle\mathrm{id}, v_{x_{0}}\right\rangle+\left\langle\mathrm{id}, v_{x_{0}}^{\infty}\right\rangle \frac{\mathrm{d} \lambda_{v}}{\mathrm{~d} \mathscr{L}^{d}}\left(x_{0}\right)\right) \leq\left\langle h, v_{x_{0}}\right\rangle+\left\langle h^{\#}, v_{x_{0}}^{\infty}\right\rangle \frac{\mathrm{d} \lambda_{v}}{\mathrm{~d} \mathscr{L}^{d}}\left(x_{0}\right),
$$

for all upper semicontinuous and $\mathscr{A}^{k}$-quasiconvex $h: \mathbb{R}^{N} \rightarrow[0, \infty)$ with linear growth at infinity.

Proof. We make use of Lemma 2.9 to get a collection $\left\{h_{m}\right\} \subset \mathbf{E}\left(\Omega ; \mathbb{R}^{N}\right)$ such that $h_{m} \downarrow h, h_{m}^{\infty} \downarrow h^{\#}$ pointwise in $\Omega$ and $\bar{\Omega}$ respectively, all $h_{m}$ are Lipschitz continuous and have uniformly bounded linear growth constants. Fix $x_{0} \in \Omega$ such that there exists a regular tangent measure $\sigma \in \mathbf{Y}_{\mathscr{A}^{k}}\left(Q ; \mathbb{R}^{N}\right)$ of $v$ at $x_{0}$ as in Proposition 2.24, which is possible for $\mathscr{L}^{d}$-a.e. $x_{0} \in \Omega$. The localization principle for regular points tells us that $[\sigma]=A_{0} \mathscr{L}^{d}$ with

$$
A_{0}:=\left\langle\mathrm{id}, v_{x_{0}}\right\rangle+\left\langle\mathrm{id}, v_{x_{0}}^{\infty}\right\rangle \frac{\mathrm{d} \lambda_{v}}{\mathrm{~d} \mathscr{L}^{d}}\left(x_{0}\right) \quad \in \mathbb{R}^{N},
$$

and that we may find a sequence $z_{j} \in \mathrm{C}_{\text {per }}^{\infty}\left(Q ; \mathbb{R}^{N}\right) \cap \operatorname{ker} \mathscr{A}^{k}$ with $\int_{Q} z_{j} \mathrm{~d} y=0$ and satisfying

$$
\left(A_{0}+z_{j}\right) \mathscr{L}^{d} \stackrel{\mathbf{Y}}{\rightarrow} \sigma \quad \text { in } \mathbf{Y}\left(Q ; \mathbb{R}^{N}\right) .
$$

Fix $m \in \mathbb{N}$. We use the fact that $\int_{Q} z_{j} \mathrm{~d} y=0,\left(\right.$ A.8) and the $\mathscr{A}^{k}$-quasiconvexity of $h$, to get for every $m \in \mathbb{N}$ that

$$
\begin{aligned}
\left\langle h_{m}, v_{x_{0}}\right\rangle+\left\langle h_{m}^{\infty}, v_{x_{0}}^{\infty}\right\rangle \frac{\mathrm{d} \lambda_{v}}{\mathrm{~d} \mathscr{L}^{d}}\left(x_{0}\right) & =\frac{1}{\left|Q_{r}\right|}\left\langle\mathbb{1}_{Q} \otimes h_{m}, \sigma\right\rangle \\
& =\lim _{j \rightarrow \infty} f_{Q} h_{m}\left(A_{0}+z_{j}(y)\right) \mathrm{d} y \\
& \geq \limsup _{j \rightarrow \infty} f_{Q} h\left(A_{0}+z_{j}(y)\right) \mathrm{d} y \\
& \geq h\left(A_{0}\right) .
\end{aligned}
$$

The result follows by letting $m \rightarrow \infty$ in the previous inequality and using the monotone convergence theorem. 
3.2. Jensen's inequality at singular points. The strategy for singular points differs from the regular case as one cannot simply use the definition of $\mathscr{A}^{k}$-quasiconvexity. The latter difficulty arises because tangent measures at a singular point may not be multiples of the $d$-dimensional Lebesgue measure.

In order to circumvent this obstacle, we will first show that, for $\mathscr{A}$-free Young measures, the support of the singular part $v^{\infty}$ at singular points is contained in the subspace $V_{\mathscr{A}}$ of $\mathbb{R}^{N}$ (see Lemma 3.2 below). Based on this, we invoke Theorem 2.21, which states that an $\mathscr{A}^{k}$-quasiconvex and positively 1-homogeneous function is actually convex at points in $\Lambda_{\mathscr{A}}$ when restricted to $V_{\mathscr{A}}$. Then, the Jensen inequality for $\mathscr{A}$-free Young measures at singular points follows.

Lemma 3.2. Let $\sigma \in \mathbf{Y}_{\mathscr{A}^{k}}\left(Q ; \mathbb{R}^{N}\right)$ be an $\mathscr{A}^{k}$-free Young measure with $\lambda_{\sigma}(\partial Q)=0$. Assume also that

$$
[\sigma] \in \mathscr{M}\left(Q ; V_{\mathscr{A}}\right)
$$

Then,

$$
\operatorname{supp} \sigma_{y}^{\infty} \subset V_{\mathscr{A}} \cap \mathbb{S}^{N-1} \quad \text { for } \lambda_{\sigma} \text {-a.e. } y \in Q \text {. }
$$

Proof. By definition, we may find a sequence $\left(\mu_{j}\right) \subset \mathscr{M}\left(Q ; \mathbb{R}^{N}\right)$ with $\mathscr{A} \mu_{j} \rightarrow 0$ in $\mathrm{W}^{-k, q}(Q)$ for some $q \in(1, d /(d-1))$, and such that $\left(\mu_{j}\right)$ generates the Young measure $\sigma$. Notice that, since $\mathscr{A}^{k}$ is a homogeneous operator and $Q$ is a strictly star-shaped domain, we may re-scale and mollify each $\mu_{j}$ into some $u_{j} \in \mathrm{L}^{2}\left(Q ; \mathbb{R}^{N}\right)$ with the following property: the sequence $\left(u_{j}\right)$ also generates $\sigma$ and $\mathscr{A} u_{j} \rightarrow 0$ in $\mathrm{W}^{-k, q}(Q)$. In particular,

$$
u_{j} \mathscr{L}^{d} \stackrel{*}{\rightarrow}[\sigma] \quad \text { in } \mathscr{M}\left(Q ; \mathbb{R}^{N}\right) .
$$

On the other hand, $\mathscr{A}^{k}([\sigma])=0$ and for every $0<r<1$ the measure $T_{\#}^{(0, r)}[\sigma]$ is still an $\mathscr{A}^{k}$-free measure on $Q$. Thus, letting $r \uparrow 1$ and mollifying the measure $T_{\#}^{(0, r)}[\sigma]$ on a sufficiently small scale (with respect to $1-r)$ we might find a sequence $\left(v_{j}\right) \subset$ $\mathrm{L}^{2}\left(Q ; \mathbb{R}^{N}\right) \cap \operatorname{ker} \mathscr{A}^{k}$ such that

$$
v_{j} \mathscr{L}^{d} \stackrel{*}{\rightarrow}[\sigma] \quad \text { in } \mathscr{M}\left(Q ; \mathbb{R}^{N}\right) .
$$

Hence,

$$
u_{j} \mathscr{L}^{d}-v_{j} \mathscr{L}^{d} \stackrel{*}{\rightarrow} 0 \quad \text { in } \mathscr{M}\left(Q ; \mathbb{R}^{N}\right), \quad\left|u_{j} \mathscr{L}^{d}\right|+\left|v_{j} \mathscr{L}^{d}\right| \stackrel{*}{\rightarrow} \Lambda \text { in } \mathscr{M}^{+}(\bar{Q})
$$

and $\Lambda(\partial Q)=0$. Here, we have used that $\lambda_{\sigma}(\partial Q)=0$.

We are now in position to apply Lemma 2.15 to the sequences $\left(u_{j}\right),\left(v_{j}\right)$. There exists (possibly passing to a subsequence in the $v_{j}$ 's) a sequence $z_{j} \in \mathrm{C}_{\text {per }}^{\infty}\left(Q ; \mathbb{R}^{N}\right) \cap$ $\operatorname{ker} \mathscr{A}^{k}$ with $z_{j} \mathscr{L}^{d} \stackrel{*}{\rightarrow} 0$ and such that

$$
v_{j} \mathscr{L}^{d}+z_{j} \mathscr{L}^{d} \stackrel{\mathbf{Y}}{\rightarrow} \sigma \quad \text { in } \mathscr{M}\left(Q ; \mathbb{R}^{N}\right) .
$$

Recall from observation (2.18) that $v_{j}, z_{j} \in \mathrm{L}^{2}\left(Q ; V_{\mathscr{A}}\right)$ for every $j \in \mathbb{N}$. Therefore,

$$
\left(v_{j}+z_{j}\right) \in \mathrm{L}^{2}\left(Q ; V_{\mathscr{A}}\right) \quad \text { for all } j \in \mathbb{N} \text {. }
$$

We conclude with an application of Lemma 2.8 (ii) to the sequence $\left(v_{j}+z_{j}\right)$, which yields

$$
\operatorname{supp} \sigma_{y}^{\infty} \subset V_{\mathscr{A}} \cap \mathbb{S}^{N-1} \quad \text { for } \lambda_{\sigma} \text {-a.e. } y \in Q \text {. }
$$

This finishes the proof. 
Proposition 3.3. Let $v \in \mathbf{Y}_{\mathscr{A}}\left(\Omega ; \mathbb{R}^{N}\right)$ be an $\mathscr{A}$-free Young measure. Then for $\lambda_{v^{-}}^{s}$ almost every $x_{0} \in \Omega$ it holds that

$$
g\left(\left\langle\mathrm{id}, v_{x_{0}}^{\infty}\right\rangle\right) \leq\left\langle g, v_{x_{0}}^{\infty}\right\rangle
$$

for all $\Lambda_{\mathscr{A}}$-convex and positively 1-homogeneous functions $g: \mathbb{R}^{N} \rightarrow \mathbb{R}$.

Proof. Step 1: Characterization of the support of $\mathscr{A}$-free Young measures. Let $S$ be the set given by Proposition 2.25, which has full $\lambda_{v}^{s}$-measure. Further, also the set

$$
S^{\prime}:=\left\{x \in \Omega:\left\langle\mathrm{id}, v_{x}^{\infty}\right\rangle \in \Lambda_{\mathscr{A}}\right\} \subset \Omega
$$

has full $\lambda_{v}^{s}$-measure: Observe first that

$$
[v]^{s}=\left\langle\mathrm{id}, v_{x}^{\infty}\right\rangle \lambda_{v}^{s}(\mathrm{~d} x) .
$$

Since $[v]$ is $\mathscr{A}$-free, we thus infer from Theorem 2.11 that $\left\langle\right.$ id, $\left.v_{x}^{\infty}\right\rangle \in \Lambda_{\mathscr{A}}$ for $\left|[v]^{s}\right|$ a.e. $x \in \Omega$. On the other hand, $\left\langle\right.$ id, $\left.v_{x}^{\infty}\right\rangle=0 \in \Lambda_{\mathscr{A}}$ for $\lambda_{v}^{*}$-a.e. $x \in \Omega$, where $\lambda_{v}^{*}$ is the singular part of $\lambda_{v}^{s}$ with respect to $\left|[v]^{s}\right|$. This shows that $S^{\prime}$ has full $\lambda_{v}^{s}$-measure.

Fix $x_{0} \in S \cap S^{\prime}$ (which remains of full $\lambda_{v}^{s}$-measure in $\Omega$ ). Let $\sigma \in \mathbf{Y}_{\mathscr{A}^{k}}\left(Q ; \mathbb{R}^{N}\right)$ be the non-zero singular tangent Young measure to $v$ at $x_{0}$ given by Proposition 2.25 which according to the same proposition satisfies that $\lambda_{\sigma}(Q)=1$ and $\lambda(\partial Q)=0$. On the one hand, since $x_{0} \in S$, it holds that

$$
\sigma_{y}=\delta_{0} \quad \mathscr{L}^{d} \text {-a.e. } \quad \text { and } \quad \sigma_{y}^{\infty}=v_{x_{0}}^{\infty} \lambda_{\sigma} \text {-a.e. }
$$

On the other hand, we use the fact that $x_{0} \in S^{\prime}$ to get

$$
\left\langle\text { id, } v_{x_{0}}^{\infty}\right\rangle \in \Lambda_{\mathscr{A}} \quad \text { and } \quad[\sigma]=\left\langle\text { id, } v_{x_{0}}^{\infty}\right\rangle \lambda_{\sigma} \in \mathscr{M}\left(Q ; V_{\mathscr{A}}\right) .
$$

Note that, by (3.2), all the hypotheses of Lemma 3.2 are satisfied for $\sigma$. Thus,

$$
\operatorname{supp} v_{x_{0}}^{\infty}=\operatorname{supp} \sigma_{y}^{\infty} \subset V_{\mathscr{A}} \quad \text { for } \lambda_{\sigma} \text {-a.e. } y \in Q \text {. }
$$

This equality and the fact that $\lambda_{\sigma}(Q)>0$ (recall that $\sigma$ is a non-zero singular measure) yield

$$
\operatorname{supp} v_{x_{0}}^{\infty} \subset V_{\mathscr{A}} \quad \text { for } \lambda_{v}^{s} \text {-a.e. } x_{0} \in \Omega \text {. }
$$

Step 2: Convexity of $g$ on $\Lambda_{\mathscr{A}}$. The Kirchheim-Kristensen Theorem 2.21 states that the restriction $\left.g\right|_{V_{\mathscr{A}}}: V_{\mathscr{A}} \subset \mathbb{R}^{N} \rightarrow \mathbb{R}$ is a convex function at points $A_{0} \in \Lambda_{\mathscr{A}}$. In other words, for every probability measure $v \in \mathscr{M}^{1}\left(\mathbb{R}^{N}\right)$ with $\langle$ id, $v\rangle \in \Lambda_{\mathscr{A}}$ and $\operatorname{supp} v \subset V_{\mathscr{A}}$, the Jensen inequality

$$
g\left(\int_{\mathbb{R}^{N}} A \mathrm{~d} v(A)\right) \leq \int_{\mathbb{R}^{N}} g(A) \mathrm{d} v(A)
$$

holds. Hence, because of (3.2) and (3.3), it follows that

$$
g\left(\left\langle\mathrm{id}, v_{x_{0}}^{\infty}\right\rangle\right) \leq\left\langle g, v_{x_{0}}^{\infty}\right\rangle .
$$

This proves the assertion.

The following simple corollary will be important in the proof of Theorem 1.7.

Corollary 3.4. Let $h: \mathbb{R}^{N} \rightarrow \mathbb{R}$ be an upper semicontinuous integrand with linear growth at infinity and let $v \in \mathbf{Y}_{\mathscr{A}}\left(\Omega ; \mathbb{R}^{N}\right)$ be an $\mathscr{A}$-free Young measure. Then, for $\mathscr{L}^{d}$-almost every $x_{0} \in \Omega$ it holds that

$$
Q_{\mathscr{A}^{k}} h\left(\left\langle\mathrm{id}, v_{x_{0}}\right\rangle+\left\langle\mathrm{id}, v_{x_{0}}^{\infty}\right\rangle \frac{\mathrm{d} \lambda_{v}}{\mathrm{~d} \mathscr{L}^{d}}\left(x_{0}\right)\right) \leq\left\langle h, v_{x_{0}}\right\rangle+\left\langle h^{\#}, v_{x_{0}}^{\infty}\right\rangle \frac{\mathrm{d} \lambda_{v}}{\mathrm{~d} \mathscr{L}^{d}}\left(x_{0}\right) .
$$


Moreover, for $\lambda_{v}^{s}$-a.e. $x_{0} \in \Omega$ it holds that

$$
\left(Q_{\mathscr{A} k} h\right)^{\#}\left(\left\langle\mathrm{id}, v_{x_{0}}^{\infty}\right\rangle\right) \leq\left\langle h^{\#}, v_{x_{0}}^{\infty}\right\rangle
$$

Proof. The proof follows by combining Propositions 3.1 and 3.3, Lemma 2.18, Corollary 2.20 and the trivial inequalities $Q_{\mathscr{A}^{k}} h \leq h,\left(Q_{\mathscr{A}^{k}} h\right)^{\#} \leq h^{\#}$.

\section{Proof of Theorems 1.2 AND 1.6}

Proof of Theorem 1.2. We will prove Theorem 1.2 in full generality, which means that we consider asymptotically $\mathscr{A}$-free sequences in the $\mathrm{W}^{-k, q}$-norm for some $q \in$ $(1, d /(d-1))$; see Remark 1.4.

Proof under Assumption (i). Let $\mu_{j}$ be a sequence in $\mathscr{M}\left(\Omega ; \mathbb{R}^{N}\right)$ weakly* converging to a limit $\mu$ and assume furthermore that $\mathscr{A} \mu_{j} \rightarrow 0$ in $\mathrm{W}^{-k, q}\left(\Omega ; \mathbb{R}^{N}\right)$ for some $q \in(1, d /(d-1))$. Up to passing to a subsequence, we might also assume that

$$
\liminf _{j \rightarrow \infty} \mathscr{F}\left[\mu_{j}\right]=\lim _{j \rightarrow \infty} \mathscr{F}\left[\mu_{j}\right]
$$

and that $\mu_{j} \stackrel{\mathbf{Y}}{\rightarrow} v$ for some $\mathscr{A}$-free Young measure $v \in \mathbf{Y}_{\mathscr{A}}\left(\Omega ; \mathbb{R}^{N}\right)$. Using the continuity of $f$ and representation of Corollary 2.10 we get

$$
\mathscr{F}\left[\mu_{j}\right]=\left\langle\left\langle f, \delta\left[\mu_{j}\right]\right\rangle \rightarrow\langle\langle f, v\rangle \quad \text { as } j \rightarrow \infty .\right.
$$

The positivity of $f$ further lets us discard possible concentration of mass on $\partial \Omega$,

$$
\begin{aligned}
& \lim _{j \rightarrow \infty} \mathscr{F}\left[\mu_{j}\right]= \int_{\bar{\Omega}}\left\langle f(x, \cdot), v_{x}\right\rangle \mathrm{d} x+\int_{\bar{\Omega}}\left\langle f^{\infty}(x, \cdot), v_{x}^{\infty}\right\rangle \mathrm{d} \lambda_{v}(x) \\
& \geq \int_{\Omega}\left(\left\langle f(x, \cdot), v_{x}\right\rangle+\left\langle f^{\infty}(x, \cdot), v_{x}^{\infty}\right\rangle \frac{\mathrm{d} \lambda_{v}}{\mathrm{~d} \mathscr{L}^{d}}(x)\right) \mathrm{d} x \\
&+\int_{\Omega}\left\langle f^{\infty}(x, \cdot), v_{x}^{\infty}\right\rangle \mathrm{d} \lambda_{v}^{s}(x) .
\end{aligned}
$$

By assumption, $f(x, \cdot) \in \mathrm{C}\left(\mathbb{R}^{N}\right)$ has linear growth at infinity. Hence we might apply Proposition 3.1 to get

$$
f\left(x,\left\langle\mathrm{id}, v_{x}\right\rangle+\left\langle\mathrm{id}, v_{x}^{\infty}\right\rangle \frac{\mathrm{d} \lambda_{v}}{\mathrm{~d} \mathscr{L}^{d}}(x)\right) \leq\left\langle f(x, \cdot), v_{x}\right\rangle+\left\langle f(x, \cdot)^{\infty}, v_{x}^{\infty}\right\rangle \frac{\mathrm{d} \lambda_{v}}{\mathrm{~d} \mathscr{L}^{d}}(x)
$$

for $\mathscr{L}^{d}$-a.e. $x \in \Omega$ (recall that under the present assumptions $f^{\infty}=f^{\#}$ ). Likewise, we apply Proposition 3.3 to the functions $f(x, \cdot)^{\#}$ to obtain

$$
f(x, \cdot)^{\infty}\left(\left\langle\mathrm{id}, v_{x}^{\infty}\right\rangle\right) \leq\left\langle f(x, \cdot)^{\infty}, v_{x}^{\infty}\right\rangle
$$

at $\lambda_{v}^{s}$-a.e. $x \in \Omega$. Plugging these two Jensen-type inequalities into (4.1) yields

$$
\begin{aligned}
\lim _{j \rightarrow \infty} \mathscr{F}\left[\mu_{j}\right] \geq \int_{\Omega} & f\left(x,\left\langle\mathrm{id}, v_{x}\right\rangle+\left\langle\mathrm{id}, v_{x}^{\infty}\right\rangle \frac{\mathrm{d} \lambda_{v}}{\mathrm{~d} \mathscr{L}^{d}}(x)\right) \mathrm{d} x \\
+ & \int_{\Omega} f^{\infty}\left(x,\left\langle\operatorname{id}_{\mathbb{R}^{N}}, v_{x}^{\infty}\right\rangle\right) \mathrm{d} \lambda_{v}^{s}(x) .
\end{aligned}
$$

Finally, since $\mu_{j} \stackrel{\mathrm{Y}}{\rightarrow} v$, it must hold that

$$
\begin{gathered}
\left\langle\mathrm{id}, v_{x}\right\rangle+\left\langle\mathrm{id}, v_{x}^{\infty}\right\rangle \frac{\mathrm{d} \lambda_{v}}{\mathrm{~d} \mathscr{L}^{d}}(x)=\frac{\mathrm{d} \mu}{\mathrm{d} \mathscr{L}^{d}}(x) \quad \text { for } \mathscr{L}^{d} \text {-a.e. } x \in \Omega, \quad \text { and } \\
\left\langle\operatorname{id}_{\mathbb{R}^{N}}, v_{x}^{\infty}\right\rangle \lambda_{v}^{s}=\mu^{s} \Rightarrow \frac{\mathrm{d} \mu^{s}}{\mathrm{~d}\left|\mu^{s}\right|}(x)=\frac{\left\langle\mathrm{id}_{\mathbb{R}^{N}}, v_{x}^{\infty}\right\rangle}{\left|\left\langle\mathrm{id}_{\mathbb{R}^{N}}, v_{x}^{\infty}\right\rangle\right|} \quad \text { for } \lambda_{v^{s}}^{s} \text {-a.e. } x \in \Omega .
\end{gathered}
$$


We can use this representation and the fact that $f^{\infty}(x, \bullet)$ is positively 1-homogeneous in the right hand side of (4.2) to conclude

$$
\begin{aligned}
& \lim _{j \rightarrow \infty} \mathscr{F}\left[\mu_{j}\right] \geq \int_{\Omega} f\left(x, \frac{\mathrm{d} \mu}{\mathrm{d} \mathscr{L}^{d}}(x)\right) \mathrm{d} x \\
&+\int_{\Omega} f^{\infty}\left(x, \frac{\mathrm{d} \mu^{s}}{\mathrm{~d}\left|\mu^{s}\right|}(x)\right) \mathrm{d}\left(\left|\left\langle\mathrm{id}_{\mathbb{R}^{N}}, v_{x}^{\infty}\right\rangle\right| \lambda_{v}^{s}\right)(x) \\
&= \int_{\Omega} f\left(x, \frac{\mathrm{d} \mu}{\mathrm{d} \mathscr{L}^{d}}(x)\right) \mathrm{d} x \\
& \quad+\int_{\Omega} f^{\infty}\left(x, \frac{\mathrm{d} \mu^{s}}{\mathrm{~d}\left|\mu^{s}\right|}(x)\right) \mathrm{d}\left|\mu^{s}\right|(x) \\
&=\mathscr{F}[\mu] .
\end{aligned}
$$

This proves the claim under Assumption (i).

Proof under Assumption (ii). For a measure $\mu \in \mathscr{M}\left(\Omega ; \mathbb{R}^{N}\right)$, consider the functional

$$
\mathscr{F}^{\#}[\mu ; B]:=\int_{B} f\left(x, \frac{\mathrm{d} \mu}{\mathrm{d} \mathscr{L}^{d}}(x)\right) \mathrm{d} x+\int_{B} f^{\#}\left(x, \frac{\mathrm{d} \mu^{s}}{\mathrm{~d}\left|\mu^{s}\right|}(x)\right) \mathrm{d}\left|\mu^{s}\right|(x),
$$

defined for any Borel subset $B \subset \Omega$.

Let $\mu_{j}$ be a sequence in $\mathscr{M}\left(\Omega ; \mathbb{R}^{N}\right)$ that weakly* converges to a limit $\mu$ and assume furthermore that $\mathscr{A} \mu_{j} \rightarrow 0$ in $\mathrm{W}^{-k, q}\left(\Omega ; \mathbb{R}^{N}\right)$ for some $q \in(1, d /(d-1))$. Define $\lambda_{j} \in \mathscr{M}^{+}(\Omega)$ via

$$
\lambda_{j}(B):=\mathscr{F}^{\#}\left[\mu_{j} ; B\right] \quad \text { for every Borel } B \subset \Omega .
$$

We may find a (not relabeled) subsequence and positive measures $\lambda, \Lambda \in \mathscr{M}_{+}(\Omega)$ such that

$$
\lambda_{j} \stackrel{*}{\rightarrow} \lambda,\left|\mu_{j}\right| \stackrel{*}{\rightarrow} \Lambda \quad \text { in } \mathscr{M}^{+}(\Omega) .
$$

We claim that

$$
\begin{array}{ll}
\frac{\mathrm{d} \lambda}{\mathrm{d} \mathscr{L}^{d}}\left(x_{0}\right) \geq f\left(x_{0}, \frac{\mathrm{d} \mu}{\mathrm{d} \mathscr{L}^{d}}\left(x_{0}\right)\right) & \text { for } \mathscr{L}^{d} \text {-a.e. } x_{0} \in \Omega, \\
\frac{\mathrm{d} \lambda}{\mathrm{d}\left|\mu^{s}\right|}\left(x_{0}\right) \geq f^{\#}\left(x_{0}, \frac{\mathrm{d} \mu^{s}}{\mathrm{~d}\left|\mu^{s}\right|}\left(x_{0}\right)\right) & \text { for }\left|\mu^{s}\right| \text {-a.e. } x_{0} \in \Omega .
\end{array}
$$

Notice that, if (4.3) and (4.4) hold, then the assertion of the theorem immediately follows. Indeed, by the Radon-Nikodým theorem,

$$
\lambda \geq \frac{\mathrm{d} \lambda}{\mathrm{d} \mathscr{L}^{d}} \mathscr{L}^{d}+\frac{\mathrm{d} \lambda}{\mathrm{d}\left|\mu^{s}\right|}\left|\mu^{s}\right| .
$$

Hence, we obtain

$$
\begin{aligned}
\liminf _{j \rightarrow \infty} \mathscr{F}^{\#}\left[\mu_{j}\right] & =\liminf _{j \rightarrow \infty} \lambda_{j}(\Omega) \\
& \geq \lambda(\Omega) \\
& \geq \int_{\Omega} \frac{\mathrm{d} \lambda}{\mathrm{d} \mathscr{L}^{d}} \mathrm{~d} x+\int_{\Omega} \frac{\mathrm{d} \lambda}{\mathrm{d}\left|\mu^{s}\right|} \mathrm{d}\left|\mu^{s}\right| \\
& \geq \int_{\Omega} f\left(x, \frac{\mathrm{d} \mu}{\mathrm{d} \mathscr{L}^{d}}(x)\right) \mathrm{d} x+\int_{\Omega} f^{\#}\left(x, \frac{\mathrm{d} \mu^{s}}{\mathrm{~d}\left|\mu^{s}\right|}(x)\right) \mathrm{d}\left|\mu^{s}\right| \\
& =\mathscr{F}^{\#}[\mu] .
\end{aligned}
$$


With (4.3), (4.4), which are proved below, the result under Assumption (ii) follows. This completes the proof of the theorem.

We now prove (4.3) and (4.4). Let us first show the following auxiliary fact.

Lemma 4.1. Let $x_{0} \in \Omega$ and $R>0$ be such that $Q_{2 R}\left(x_{0}\right) \subset \Omega$. Then, for every $h \in \mathbb{N}$, there exists a sequence $\left(u_{j}^{h}\right) \subset \mathrm{L}^{2}\left(\mathbb{R}^{d} ; \mathbb{R}^{N}\right)$ such that

$$
\begin{aligned}
& u_{j}^{h} \rightarrow \mu_{j} \quad \text { area-strictly in } \mathscr{M}\left(Q_{R}\left(x_{0}\right) ; \mathbb{R}^{N}\right) \quad \text { and } \\
& \left\|\mathscr{A}^{k} u_{j}^{h}-\mathscr{A}^{k} \mu_{j}\right\|_{\mathrm{W}^{-k, q}\left(Q_{R}\left(x_{0}\right)\right)} \rightarrow 0 . \quad \text { as } \quad h \rightarrow \infty
\end{aligned}
$$

Proof. Let $\left\{\rho_{\varepsilon}\right\}_{\varepsilon>0}$ be a family of standard smooth mollifiers. The sequence defined by

$$
u_{j}^{h}:=\left(\mu_{j}\left\llcorner Q_{3 R / 2}\left(x_{0}\right)\right) * \rho_{1 / h} \in \mathrm{C}^{\infty}\left(\overline{Q_{2 R}\left(x_{0}\right)} ; \mathbb{R}^{N}\right)\right.
$$

satisfies all the conclusion properties as a consequence of the properties of mollification and Remark 2.2

Proof of (4.3). We employ the classical blow-up method to organize the proof. We know from Lebesgue's differentiation theorem and (2.5) that the following properties hold for $\mathscr{L}^{d}$-almost every $x_{0}$ in $\Omega$ :

$$
\begin{gathered}
\frac{\mathrm{d} \lambda}{\mathrm{d} \mathscr{L}^{d}}\left(x_{0}\right)=\lim _{r \downarrow 0} \frac{\lambda\left(Q_{r}\left(x_{0}\right)\right)}{r^{d}}<\infty, \quad \lim _{r \downarrow 0} \frac{\left|\mu^{s}\right|\left(Q_{r}\left(x_{0}\right)\right)}{r^{d}}<\infty, \\
\lim _{r \downarrow 0} \frac{1}{r^{d}} \int_{Q_{r}\left(x_{0}\right)}\left|\frac{\mathrm{d} \mu}{\mathrm{d} \mathscr{L}^{d}}(y)-\frac{\mathrm{d} \mu}{\mathrm{d} \mathscr{L}^{d}}\left(x_{0}\right)\right| \mathrm{d} y=0, \\
\lim _{r \downarrow 0} \frac{1}{r^{d}} \int_{Q_{r}\left(x_{0}\right)}\left|\frac{\mathrm{d} \Lambda}{\mathrm{d} \mathscr{L}^{d}}(y)-\frac{\mathrm{d} \Lambda}{\mathrm{d} \mathscr{L}^{d}}\left(x_{0}\right)\right| \mathrm{d} y=0,
\end{gathered}
$$

and

$$
\operatorname{Tan}\left(\mu, x_{0}\right)=\left\{\alpha \cdot \frac{\mathrm{d} \mu}{\mathrm{d} \mathscr{L}^{d}}\left(x_{0}\right) \mathscr{L}^{d}: \alpha \in \mathbb{R}^{+} \cup\{0\}\right\}
$$

Let $x_{0} \in \Omega$ be a point where the properties above are satisfied. Since $\Omega$ is an open set, there exists a positive number $R$ such that $Q_{2 R\left(x_{0}\right)} \subset \Omega$. From Lemma 4.1, we infer that for almost every $r \in(0, R)$, it holds that

$$
\begin{aligned}
& \mathrm{w}_{j \rightarrow \infty}^{*} \lim _{h \rightarrow \infty} \mathrm{w}^{*}-\lim \left[u_{j}^{h}\left(x_{0}+r y\right) \mathscr{L}_{y}^{d}\right]=\mathrm{w}_{j \rightarrow \infty}^{*-\lim } \mathrm{w}_{h \rightarrow \infty}^{*-\lim } r^{-d} T_{\#}^{\left(x_{0}, r\right)}\left[u_{j}^{h} \mathscr{L}^{d}\right] \\
& =\mathrm{w}_{j \rightarrow \infty}^{*}-\lim ^{-d} r_{\#}^{\left(x_{0}, r\right)} \mu_{j} \\
& =r^{-d} T_{\#}^{\left(x_{0}, r\right)} \mu \text {, }
\end{aligned}
$$

where the weak* convergence is to be understood in $\mathscr{M}\left(Q ; \mathbb{R}^{N}\right)$. Thus, choosing a sequence $r \downarrow 0$ with $\lambda_{j}\left(\partial Q_{r}\left(x_{0}\right)\right)=0$ and $\Lambda\left(\partial Q_{r}\left(x_{0}\right)\right)=0$ (by the finiteness of these 
measures), we get that

$$
\begin{aligned}
& \frac{\mathrm{d} \lambda}{\mathrm{d} \mathscr{L}^{d}}\left(x_{0}\right)=\lim _{r \rightarrow 0} \lim _{j \rightarrow \infty} \frac{\lambda_{j}\left(Q_{r}\left(x_{0}\right)\right)}{r^{d}} \\
& =\lim _{r \rightarrow 0} \lim _{j \rightarrow \infty} \frac{\mathscr{F}^{\#}\left[\mu_{j} ; Q_{r}\left(x_{0}\right)\right]}{r^{d}} \\
& \geq \limsup _{r \rightarrow 0} \limsup _{j \rightarrow \infty} \limsup _{h \rightarrow \infty} \frac{\mathscr{F}^{\#}\left[u_{j}^{h} \mathscr{L}^{d} ; Q_{r}\left(x_{0}\right)\right]}{r^{d}} \\
& =\limsup _{r \rightarrow 0} \limsup _{j \rightarrow \infty} \limsup _{h \rightarrow \infty} \int_{Q} f\left(x_{0}+r y, u_{j}^{h}\left(x_{0}+r y\right)\right) \mathrm{d} y,
\end{aligned}
$$

where we used Corollary 2.10 and Remark 2.6 for the " $\geq$ " estimate.

Moreover, by the Lebesgue differentiation theorem (see (4.7)),

$$
r^{-d} T_{\#}^{\left(x_{0}, r\right)} \mu \stackrel{*}{\rightarrow} \frac{\mathrm{d} \mu}{\mathrm{d} \mathscr{L}^{d}}\left(x_{0}\right) \mathscr{L}^{d} .
$$

By (4.8), (4.9), (4.10) and a suitable diagonalization procedure (recall that all measures involved have locally uniformly bounded variation), we can find sequences $r_{m} \downarrow 0, j_{m} \rightarrow \infty, h_{m} \rightarrow \infty$ (as $\left.m \rightarrow \infty\right)$ such that for

$$
u_{m}:=u_{j_{m}}^{h_{m}} \quad \text { and } \quad \gamma_{m}:=r_{m}^{-d} T_{\#}^{\left(x_{0}, r_{m}\right)}\left[u_{m} \mathscr{L}^{d}\right]
$$

it holds that

(1) $\gamma_{m} * \frac{\mathrm{d} \mu}{\mathrm{d} \mathscr{L}^{d}}\left(x_{0}\right) \mathscr{L}^{d}$;

(2) $\frac{\mathrm{d} \lambda}{\mathrm{d} \mathscr{L}^{d}}\left(x_{0}\right) \geq \lim _{m \rightarrow \infty} \int_{Q} f\left(x_{0}+r_{m} y, \frac{\mathrm{d} \gamma_{m}}{\mathrm{~d} \mathscr{L}^{d}}(y)\right) \mathrm{d} y$.

By Proposition 2.16 and the first property,

$$
\begin{aligned}
& \gamma_{m}-\frac{\mathrm{d} \mu}{\mathrm{d} \mathscr{L}^{d}}\left(x_{0}\right) \mathscr{L}^{d} \stackrel{*}{\rightarrow} 0 \quad \text { in } \mathscr{M}\left(Q ; \mathbb{R}^{N}\right), \quad \text { and } \\
& \mathscr{A}^{k}\left(\gamma_{m}-\frac{\mathrm{d} \mu}{\mathrm{d} \mathscr{L}^{d}}\left(x_{0}\right) \mathscr{L}^{d}\right) \rightarrow 0 \quad \text { in } \mathrm{W}^{-k, q}\left(Q ; \mathbb{R}^{N}\right) .
\end{aligned}
$$

We are now in a position to apply Lemma 2.15 to the sequence $\gamma_{m}$ and the Lipschitz function $f\left(x_{0}, \bullet\right)$, whence there exists a sequence $\left(z_{m}\right) \subset \mathrm{C}_{\text {per }}^{\infty}\left(Q ; \mathbb{R}^{N}\right)$ such that

$$
\mathscr{A} z_{m}=0, \quad \int_{Q} z_{m}=0, \quad z_{m} \stackrel{*}{*} 0 \quad \text { in } \mathscr{M}\left(Q ; \mathbb{R}^{N}\right)
$$

and

$$
\liminf _{m \rightarrow \infty} \int_{Q} f\left(x_{0}, \frac{\mathrm{d} \gamma_{m}}{\mathrm{~d} \mathscr{L}^{d}}(y)\right) \mathrm{d} y \geq \liminf _{m \rightarrow \infty} \int_{Q} f\left(x_{0}, \frac{\mathrm{d} \mu}{\mathrm{d} \mathscr{L}^{d}}\left(x_{0}\right)+z_{m}(y)\right) \mathrm{d} y .
$$


Hence, using the second property above and our assumption (1.4) on the integrand, we have

$$
\begin{aligned}
\frac{\mathrm{d} \lambda}{\mathrm{d} \mathscr{L}^{d}}\left(x_{0}\right) & \geq \lim _{m \rightarrow \infty} \int_{Q} f\left(x_{0}+r_{m} y, \frac{\mathrm{d} \gamma_{m}}{\mathrm{~d} \mathscr{L}^{d}}(y)\right) \mathrm{d} y \\
& =\lim _{m \rightarrow \infty} \int_{Q} f\left(x_{0}, \frac{\mathrm{d} \gamma_{m}}{\mathrm{~d} \mathscr{L}^{d}}(y)\right) \mathrm{d} y \\
& \geq \liminf _{m \rightarrow \infty} \int_{Q} f\left(x_{0}, \frac{\mathrm{d} \mu}{\mathrm{d} \mathscr{L}^{d}}\left(x_{0}\right)+z_{m}(y)\right) \\
& \geq f\left(x_{0}, \frac{\mathrm{d} \mu}{\mathrm{d} \mathscr{L}^{d}}\left(x_{0}\right)\right) .
\end{aligned}
$$

This proves (4.3).

Remark 4.2. If the assumption that $f(x, \bullet)$ is $\mathscr{A}^{k}$-quasiconvex is dropped, one can still show that

$$
\frac{\mathrm{d} \lambda}{\mathrm{d} \mathscr{L}^{d}}\left(x_{0}\right) \geq Q_{\mathscr{A}^{k}} f\left(x_{0}, \frac{\mathrm{d} \mu}{\mathrm{d} \mathscr{L}^{d}}\left(x_{0}\right)\right) .
$$

Indeed, the $\mathscr{A}^{k}$-quasiconvexity of $f(x, \cdot)$ has only been used in the last inequality of (4.11) where one can first use the inequality $f(x, \cdot) \geq Q_{\mathscr{A}^{k}} f(x, \cdot)$ to get

$$
\int_{Q} f\left(x_{0}, \frac{\mathrm{d} \mu}{\mathrm{d} \mathscr{L}^{d}}\left(x_{0}\right)+z_{r}(y)\right) \geq \int_{Q} Q_{\mathscr{A}^{k}} f\left(x_{0}, \frac{\mathrm{d} \mu}{\mathrm{d} \mathscr{L}^{d}}\left(x_{0}\right)+z_{r}(y)\right) .
$$

which follows by the very definition of $Q_{\mathscr{A}^{k}} f(x, \bullet)$.

Proof of (4.4). Passing to a subsequence if necessary, we may assume that

$$
\mu_{j} \stackrel{\mathbf{Y}}{\rightarrow} v \quad \text { for some } v \in \mathbf{Y}_{\mathscr{A}}\left(\Omega ; \mathbb{R}^{N}\right) .
$$

For each $j \in \mathbb{N}$ set $v_{j}:=\delta\left[\mu_{j}\right] \in \mathbf{Y}\left(\Omega ; \mathbb{R}^{N}\right)$, the elementary Young measure corresponding to $\mu_{j}$, so that $v_{j} \stackrel{*}{\rightarrow} v$ in $\mathbf{Y}\left(\Omega ; \mathbb{R}^{N}\right)$. Define the functional

$$
\mathscr{F}_{\#}[\sigma ; B]:=\int_{B}\left\langle f(x, \cdot), \sigma_{x}\right\rangle \mathrm{d} x+\int_{\bar{B}}\left\langle f_{\#}(x, \cdot), \sigma_{x}^{\infty}\right\rangle \mathrm{d} \lambda_{v}(x), \quad \sigma \in \mathbf{Y}\left(\Omega ; \mathbb{R}^{N}\right),
$$

where $B \subset \Omega$ is an open set. Observe that, as a functional defined on $\mathbf{Y}\left(\Omega ; \mathbb{R}^{N}\right), \mathscr{F}_{\#}$ is sequentially weakly* lower semicontinuous (see Corollary 2.10). We use Assumption (ii), which is equivalent to

$$
f^{\#}(x, \cdot) \equiv f_{\#}(x, \cdot) \quad \text { on } V_{\mathscr{A}},
$$

and the fact, proved in (3.3), that

$$
\operatorname{supp} v_{x}^{\infty} \subset V_{\mathscr{A}} \text { for } \lambda_{v}^{s} \text {-a.e. } x \in \Omega,
$$

to get (recall $f \geq 0$ )

$$
\begin{aligned}
\liminf _{j \rightarrow \infty} \mathscr{F}^{\#}\left[\mu_{j} ; B\right] \geq & \liminf _{j \rightarrow \infty} \mathscr{F}_{\#}\left[v_{j} ; B\right] \\
\geq & \mathscr{F}_{\#}[v ; B] \\
\geq & \int_{B}\left(\left\langle f(x, \cdot), v_{x}\right\rangle+\left\langle f_{\#}(x, \cdot), v_{x}^{\infty}\right\rangle \frac{\mathrm{d} \lambda_{v}}{\mathrm{~d} \mathscr{L}^{d}}(x)\right) \mathrm{d} x \\
& \quad+\int_{B}\left\langle f_{\#}(x, \cdot), v_{x}^{\infty}\right\rangle \mathrm{d} \lambda_{v}^{s}(x) \\
\geq & \int_{B}\left\langle f^{\#}(x, \cdot), v_{x}^{\infty}\right\rangle \mathrm{d} \lambda_{v}^{s}(x) .
\end{aligned}
$$


Recall that, for every $x \in \Omega$, the function $f(x, \bullet)$ is $\mathscr{A}^{k}$-quasiconvex and hence the function $f^{\#}(x, \bullet)$ is $\Lambda_{\mathscr{A}}$-convex and positively 1-homogeneous. An application of the Jensen-type inequality from Proposition 3.3 to the last line yields

$$
\liminf _{j \rightarrow \infty} \mathscr{F}^{\#}\left[\mu_{j} ; B\right] \geq \int_{B} f^{\#}\left(x,\left\langle\mathrm{id}, v_{x}^{\infty}\right\rangle\right) \mathrm{d} \lambda_{v}^{s}(x) .
$$

Thus, also taking into account $\left|\mu^{s}\right|=\left|\left\langle\mathrm{id}, v_{x}^{\infty}\right\rangle\right| \lambda_{v}^{s}$ and $f^{\#}\left(x,\left\langle\mathrm{id}, v_{x}^{\infty}\right\rangle\right)=f^{\#}(x, 0)=0$ for $\lambda_{v}^{*}$-a.e. $x \in \Omega$, where $\lambda_{v}^{*}$ is the singular part of $\lambda_{v}^{s}$ with respect to $\left|\mu^{s}\right|$, we get

$$
\lambda(B) \geq \int_{B} f^{\#}\left(x, \frac{\mathrm{d} \mu^{s}}{\mathrm{~d}\left|\mu^{s}\right|}(x)\right) \mathrm{d}\left|\mu^{s}\right|(x),
$$

for all open sets $B \subset \Omega$ with $\lambda_{v}^{s}(\partial B)=0$. Therefore, by the Besicovitch differentiation theorem and using the continuity of $f$ (see (1.4)) in its first argument we get

$$
\frac{\mathrm{d} \lambda}{\mathrm{d}\left|\mu^{s}\right|}\left(x_{0}\right) \geq f^{\#}\left(x_{0}, \frac{\mathrm{d} \mu^{s}}{\mathrm{~d}\left|\mu^{s}\right|}\left(x_{0}\right)\right) \quad \text { for }\left|\mu^{s}\right| \text {-a.e. } x_{0} \in \Omega \text {. }
$$

This proves (4.4).

Remark 4.3 (recession functions). The only part of the proof where we use the existence of $f^{\infty}(x, A)$, for $x \in \Omega$ and $A \in V_{\mathscr{A}}$, is in showing that

$$
\begin{aligned}
\mathscr{F}_{\#}[v ; B] \geq \int_{B} & \left(\left\langle f(x, \cdot), v_{x}\right\rangle+\left\langle f_{\#}(x, \cdot), v_{x}^{\infty}\right\rangle \frac{\mathrm{d} \lambda_{v}}{\mathrm{~d} \mathscr{L}^{d}}(x)\right) \mathrm{d} x \\
& +\int_{B}\left\langle f^{\#}(x, \cdot), v_{x}^{\infty}\right\rangle \mathrm{d} \lambda_{v}^{s}(x)
\end{aligned}
$$

The need of such an estimate comes from the fact that, in general, it is unknown whether $f_{\#}$ is a $\Lambda_{\mathscr{A}}$-convex function.

Remark 4.4. If we drop the assumption that $f(x, \cdot)$ is $\mathscr{A}^{k}$-quasiconvex for every $x \in \Omega$, we can still show that

$$
\int_{\Omega} Q_{\mathscr{A}^{k}} f\left(x, \frac{\mathrm{d} \mu}{\mathrm{d} \mathscr{L}^{d}}(x)\right) \mathrm{d} x+\int_{\Omega}\left(Q_{\mathscr{A}^{k}} f\right)^{\#}\left(x, \frac{\mathrm{d} \mu^{s}}{\mathrm{~d}\left|\mu^{s}\right|}(x)\right) \mathrm{d}\left|\mu^{s}\right|(x) \leq \liminf _{j \rightarrow \infty} \mathscr{F}\left[\mu_{j}\right]
$$

for every sequence $\mu_{j} \stackrel{*}{\rightarrow} \mu$ in $\mathscr{M}\left(\Omega ; \mathbb{R}^{N}\right)$ such that $\mathscr{A} \mu_{j} \rightarrow 0$ in $\mathrm{W}^{-k, q}(\Omega)$. The proof of this fact follows directly from Remark 4.2, the last line of (4.12) together with the continuity of $f$ in its first argument (for the Besicovitch differentiation arguments), and Corollary 3.4. Observe that one does not require the existence of $\left(Q_{\mathscr{A}^{k}} f\right)^{\infty}$ in $\Omega \times \operatorname{span} \Lambda_{\mathscr{A}}$.

Proof of Theorem 1.6. Note that in the proof of (4.3) we did not use that $f^{\infty}$ exists in $\Omega \times \operatorname{span} \Lambda_{\mathscr{A}}$. By the very same argument as in (4.5), is easy to check that Theorem 1.6 is an immediate consequence of (4.3).

\section{Proof of Theorems 1.7 AND 1.8}

We use standard machinery to show the relaxation theorems. Recall that, for Theorems 1.7 and 1.8 , we assume that $\mathscr{A}$ is a homogeneous partial differential operator. 
5.1. Proof of Theorem 1.7. Step 1. The lower bound. The lower bound $\bar{G} \geq \mathscr{G}_{*}$, where

$$
\mathscr{G}_{*}[\mu]:=\int_{\Omega} Q_{\mathscr{A}} f\left(x, \frac{\mathrm{d} \mu}{\mathrm{d} \mathscr{L}^{d}}(x)\right) \mathrm{d} x+\int_{\Omega}\left(Q_{\mathscr{A}} f\right)^{\#}\left(x, \frac{\mathrm{d} \mu^{s}}{\mathrm{~d}\left|\mu^{s}\right|}(x)\right) \mathrm{d}\left|\mu^{s}\right|(x),
$$

is a direct consequence of Remark 4.4 and the fact that $\mathscr{A}$ is a homogeneous partial differential operator $\left(\mathscr{A}=\mathscr{A}^{k}\right)$.

We divide the proof of the upper bound in Theorem 1.7 into several steps. First, we prove that any $\mathscr{A}$-free measure may be area-strictly approximated by asymptotically $\mathscr{A}$-free absolutely continuous measures. Next, we prove the upper bound on absolutely continuous measures, from which the general upper bound follows by approximation.

Step 2. An area-strictly converging recovery sequence. Let $\mu \in \mathscr{M}\left(\Omega ; \mathbb{R}^{N}\right) \cap$ $\operatorname{ker} \mathscr{A}$. We will show that there exists a sequence $\left(u_{j}\right) \subset \mathrm{L}^{1}\left(\Omega ; \mathbb{R}^{N}\right)$ for which

$$
\begin{gathered}
u_{j} \mathscr{L}^{d} \stackrel{*}{\rightarrow} \mu \quad \text { in } \mathscr{M}\left(\Omega ; \mathbb{R}^{N}\right), \quad\left\langle u_{j} \mathscr{L}^{d}\right\rangle(\Omega) \rightarrow\langle\mu\rangle(\Omega), \\
\text { and } \quad \mathscr{A} u_{j} \rightarrow 0 \quad \text { in } \mathrm{W}^{-k, q}(\Omega) .
\end{gathered}
$$

Let $\left\{\varphi_{i}\right\}_{i \in \mathbb{N}} \subset \mathrm{C}_{c}^{\infty}(\Omega)$ be a locally finite partition of unity of $\Omega$. Set

$$
\mu_{(i)}:=\mu \varphi_{i} \in \mathscr{M}\left(\Omega ; \mathbb{R}^{N}\right),
$$

and

$$
\mu_{(i)}^{a}:=\mu^{a} \varphi_{i} \quad \mu_{(i)}^{s}:=\mu^{s} \varphi_{i}
$$

where, as usual,

$$
\mu^{a}=\frac{\mathrm{d} \mu}{\mathrm{d} \mathscr{L}^{d}} \mathscr{L}^{d} \quad \text { and } \quad \mu^{s}=\mu-\mu^{a} .
$$

Note that, with a slight abuse of notation,

$$
\left\|\sum_{i=1}^{j} \mu_{(i)}^{a}-\mu^{a}\right\|_{\mathrm{L}^{1}(\Omega)} \rightarrow 0 \quad \text { as } j \rightarrow \infty .
$$

Furthermore, for fixed $i$,

$$
\left(\mu_{(i)} * \rho_{\varepsilon}\right) \mathscr{L}^{d} \stackrel{*}{\rightarrow} \mu_{(i)}, \quad\left|\mu_{(i)} * \rho_{\varepsilon}\right|(\Omega) \leq\left|\mu_{(i)}\right|(\Omega)=\int_{\Omega} \varphi_{i} \mathrm{~d}|\mu|,
$$

and

$$
\mu_{(i)}^{a} * \rho_{\varepsilon} \rightarrow \mu_{(i)}^{a} \quad \text { in } \mathrm{L}^{1}(\Omega) \quad \text { as } \varepsilon \rightarrow 0
$$

Moreover,

$$
\mathscr{A}\left(\mu_{(i)} * \rho_{\varepsilon}\right) \rightarrow \mathscr{A} \mu_{(i)} \quad \text { in } \mathrm{W}^{-k, q}(\Omega) \quad \text { as } \varepsilon \rightarrow 0 .
$$

Fix $j \in \mathbb{N}$. From (5.1) and the convergence above we might find a sequence $\varepsilon_{i}(j) \downarrow$ 0 such that the measures $\mu_{i, j}:=\mu_{(i)} * \rho_{\varepsilon_{i}(j)}$ and $\mu_{i, j}^{a}:=\mu_{(i)}^{a} * \rho_{\varepsilon_{i}(j)}$ verify

$$
\begin{gathered}
d\left(\mu_{i, j} \mathscr{L}^{d}, \mu_{(i)}\right) \leq \frac{1}{2^{i} j}, \\
\left\|\mu_{i, j}^{a}-\mu_{(i)}^{a}\right\|_{\mathrm{L}^{1}(\Omega)} \leq \frac{1}{2^{i} j}, \\
\left\|\mu_{i, j}-\mu_{(i)}\right\|_{\mathrm{W}^{-k, q}(\Omega)} \leq \frac{1}{2^{i} j},
\end{gathered}
$$


where $d$ is the metric inducing the weak* convergence on a suitable subset of $\mathscr{M}\left(\Omega ; \mathbb{R}^{N}\right)$ (the existence of the metric $d$ is a standard result for the duals of separable Banach spaces). Define the integrable functions (identifying $\mu_{i, j}^{a}$ with its density)

$$
u_{j}:=\sum_{i=1}^{\infty} \mu_{i, j}, \quad u_{j}^{a}:=\sum_{i=1}^{\infty} \mu_{i, j}^{a}
$$

We get

$$
d\left(u_{j} \mathscr{L}^{d}, \mu\right) \leq \sum_{i=1}^{\infty} d\left(\mu_{i, j} \mathscr{L}^{d}, \mu_{(i)}\right) \leq \sum_{i=1}^{\infty} \frac{1}{2^{i} j}=\frac{1}{j},
$$

and in a similar way

$$
\begin{aligned}
\left\|u_{j}^{a}-\mu^{a}\right\|_{L^{1}(\Omega)} & \leq \frac{1}{j}, \\
\left\|\mathscr{A} u_{j}\right\|_{\mathrm{W}^{-k, q}(\Omega)} & \leq \frac{1}{j},
\end{aligned}
$$

where we use that $\mu$ is $\mathscr{A}$-free in the second inequality. Observe that (5.1) and the fact that $\left\{\varphi_{i}\right\}_{i \in \mathbb{N}}$ is a partition of unity imply

$$
\int_{\Omega}\left|u_{j}\right| \mathrm{d} x \leq \sum_{i=1}^{\infty} \int_{\Omega} \varphi_{i} \mathrm{~d}|\mu| \leq|\mu|(\Omega) .
$$

Therefore $\left\|u_{j}\right\|_{\mathrm{L}^{1}(\Omega)}$ is uniformly bounded and hence

$$
\begin{gathered}
u_{j} \mathscr{L}^{d} \stackrel{*}{\rightarrow} \mu \quad \text { in } \mathscr{M}\left(\Omega ; \mathbb{R}^{N}\right), \\
\left\|u_{j}^{a}-\mu^{a}\right\|_{\mathrm{L}^{1}(\Omega)} \rightarrow 0, \\
\left\|\mathscr{A} u_{j}\right\|_{\mathrm{W}^{-k, q}(\Omega)} \rightarrow 0
\end{gathered}
$$

as $j \rightarrow \infty$. Moreover, the weak* lower semicontinuity of the total variation and (5.2) imply the strict convergence

$$
\left|u_{j} \mathscr{L}^{d}\right|(\Omega) \rightarrow|\mu|(\Omega) .
$$

Thanks to (5.3) and (5.5), to conclude the proof of the claim it suffices to show that

$$
\lim _{j \rightarrow \infty}\left\langle u_{j} \mathscr{L}^{d}\right\rangle(\Omega)=\langle\mu\rangle(\Omega) .
$$

Exploiting (5.3), (5.4), (5.6), we get

$$
\int_{\Omega}\left|u_{j}-u_{j}^{a}\right| \mathrm{d} x \rightarrow\left|\mu^{s}\right|(\Omega) \quad \text { as } j \rightarrow \infty .
$$

By the inequality $\sqrt{1+|z|^{2}} \leq \sqrt{1+|z-w|^{2}}+|w|\left(\right.$ for $z, w \in \mathbb{R}^{N}$ ), we get

$$
\left\langle u_{j} \mathscr{L}^{d}\right\rangle(\Omega) \leq\left\langle u_{j}^{a} \mathscr{L}^{d}\right\rangle(\Omega)+\int_{\Omega}\left|u_{j}-u_{j}^{a}\right| \mathrm{d} x .
$$

Hence, again by (5.4) and (5.8)

$$
\limsup _{j \rightarrow \infty}\left\langle u_{j} \mathscr{L}^{d}\right\rangle(\Omega) \leq\langle\mu\rangle(\Omega) .
$$

On the other hand, by the weak* convergence $u_{j} \mathscr{L}^{d} \stackrel{*}{\rightarrow} \mu$ and the convexity of $z \mapsto$ $\sqrt{1+|z|^{2}}$,

$$
\liminf _{j \rightarrow \infty}\left\langle u_{j} \mathscr{L}^{d}\right\rangle(\Omega) \geq\langle\mu\rangle(\Omega) .
$$

Thus, together with (5.9), (5.7) follows, concluding the proof of the claim. 
Step 3.a. Upper bound on absolutely continuous fields. Let us now turn to the derivation of the upper bound for $\overline{\mathscr{G}}[u]=\overline{\mathscr{G}}\left[u \mathscr{L}^{d}\right]$ where $u \in \mathrm{L}^{1}\left(\Omega ; \mathbb{R}^{N}\right) \cap \operatorname{ker} \mathscr{A}$. For now let us assume additionally the following strengthening of (1.4):

$$
f(x, A)-f(y, A) \leq \omega(|x-y|)(1+f(y, A)) \quad \text { for all } x, y \in \Omega, A \in \mathbb{R}^{N} .
$$

It holds that $Q_{\mathscr{A}^{k}} f(x, \cdot)$ is still uniformly Lipschitz in the second variable and

$$
Q_{\mathscr{A}} f(x, A) \leq Q_{\mathscr{A}} f(y, A)+\omega(|x-y|)(1+|A|)
$$

for every $x, y \in \Omega$ and $A \in \mathbb{R}^{N}$ with a new modulus of continuity (still denoted by $\omega$ ), which incorporates another multiplicative constant in comparison to the original $\omega$. Indeed, fix $x, y \in \Omega, \varepsilon>0$, and $A \in \mathbb{R}^{N}$. Let $w \in \mathrm{C}_{\text {per }}^{\infty}\left(Q ; \mathbb{R}^{N}\right) \cap \operatorname{ker} \mathscr{A}$ be a function with zero mean in $Q$ such that

$$
\int_{Q} f(y, A+w(z)) \mathrm{d} z \leq Q_{\mathscr{A}} f(y, A)+\varepsilon .
$$

By assumption, we get

$$
\begin{aligned}
\int_{Q} f(x, A+w(z)) \mathrm{d} z \leq & \int_{Q} f(y, A+w(z)) \mathrm{d} z \\
& +\omega(|x-y|)\left(1+\int_{Q} f(y, A+w(z)) \mathrm{d} z\right) \\
\leq & Q_{\mathscr{A}} f(y, A)+\varepsilon \\
& +\omega(|x-y|)\left(1+Q_{\mathscr{A}} f(y, A)+\varepsilon\right) .
\end{aligned}
$$

Thus,

$$
Q_{\mathscr{A}} f(x, A) \leq Q_{\mathscr{A}} f(y, A)+\varepsilon+\omega(|x-y|)\left(1+Q_{\mathscr{A}} f(y, A)+\varepsilon\right) .
$$

The linear growth at infinity of $f$, which is inherited by $Q_{\mathscr{A}} f$, gives

$$
Q_{\mathscr{A}} f(x, A) \leq Q_{\mathscr{A}} f(y, A)+\omega(|x-y|)(1+M(1+|A|))+\varepsilon(1+\omega(|x-y|)) .
$$

We may now let $\varepsilon \downarrow 0$ in the previous inequality to obtain

$$
Q_{\mathscr{A}} f(x, A) \leq Q_{\mathscr{A}} f(y, A)+\omega(|x-y|)(M+1)(1+|A|) .
$$

This proves (5.11) provided that (5.10) holds.

Fix $m \in \mathbb{N}$ and consider a partition of $\mathbb{R}^{d}$ of cubes of side length $1 / m$. Let $\left\{Q_{i}^{m}\right\}_{i=1}^{L(m)}$ be the maximal collection of those cubes (with centers $\left\{x_{i}^{m}\right\}_{i=1}^{L(m)}$ ) that are compactly contained in $\Omega$. We have

$$
\mathscr{L}^{d}(\Omega)=\sum_{i=1}^{L(m)} \mathscr{L}^{d}\left(Q_{i}^{h}\right)+\mathrm{o}_{m}(1),
$$

where $\mathrm{o}_{m}(1) \rightarrow 0$ as $m \rightarrow \infty$.

We may approximate $u$ strongly in $\mathrm{L}^{1}$ by functions $z^{m} \in \mathrm{L}^{1}\left(\Omega ; \mathbb{R}^{N}\right)$ that are piecewise constant on the mesh $\left\{Q_{i}^{m}\right\}_{i=1}^{L(m)}$ (as $m \rightarrow \infty$ ). More specifically, we may find functions $z^{m} \in \mathrm{L}^{1}\left(\Omega ; \mathbb{R}^{N}\right)$ such that $z^{m}=0$ on $\Omega \backslash \bigcup_{i} Q_{i}^{m}$,

$$
z^{m}=z_{i}^{m} \in \mathbb{R}^{N} \quad \text { on } Q_{i}^{m} \quad \text { and } \quad\left\|u-z^{m}\right\|_{\mathrm{L}^{1}(\Omega)}=\mathrm{o}_{m}(1) .
$$

Additionally, for every $m \in \mathbb{N}$, we may find functions $w_{i}^{m} \in \mathrm{C}_{\text {per }}^{\infty}\left(Q ; \mathbb{R}^{N}\right) \cap \operatorname{ker} \mathscr{A}$ with the properties

$$
\int_{Q} f\left(x_{i}^{m}, z_{i}^{m}+w_{i}^{m}(y)\right) \mathrm{d} y \leq Q_{\mathscr{A}} f\left(x_{i}^{m}, z_{i}^{m}\right)+\frac{1}{m}, \quad \int_{Q} w_{i}^{m} \mathrm{~d} y=0 .
$$


Fix $m \in \mathbb{N}$ and let $\varphi_{m} \in \mathrm{C}_{c}^{\infty}(Q ;[0,1])$ be a function such that

$$
\sum_{i=1}^{L(m)}\left\|1-\varphi_{m}\right\|_{\mathrm{L}^{1}(Q)}\left\|w_{i}^{m}\right\|_{\mathrm{L}^{1}(Q)} \leq \frac{1}{m}
$$

We define the functions

$$
v_{j}^{m}:=\sum_{i=1}^{L(m)} \varphi_{m}\left(m\left(x-x_{i}^{m}\right)\right) \cdot w_{i}^{m}\left(j m\left(x-x_{i}^{m}\right)\right) \quad x \in \Omega, j \in \mathbb{N} .
$$

By Lemma 2.7, the sequence $\left(v_{j}^{m}\right)_{j}$ generates the Young measure

$$
v^{m}=\left(v_{x}^{m}, 0, \cdot\right) \in \mathbf{Y}\left(\Omega ; \mathbb{R}^{N}\right),
$$

where for each $x \in \Omega, v_{x}^{m}$ is the probability measure defined by duality trough

$$
\left\langle h, v_{x}^{m}\right\rangle:=\sum_{i=1}^{L(m)} \mathbb{1}_{Q_{i}^{m}}(x) \int_{Q} h\left(\varphi_{m}\left(m\left(x-x_{i}^{m}\right)\right) \cdot w_{i}^{m}(y)\right) \mathrm{d} y,
$$

on functions $h \in \mathrm{C}\left(\mathbb{R}^{N}\right)$ with linear growth.

The central point of this construction is that $w_{i}^{m}$ has zero mean value, that is, $\int_{Q} w_{i}^{m} \mathrm{~d} y=0$, whereby it follows that

$$
v_{j}^{m} \mathscr{L}^{d} \quad \stackrel{*}{\rightarrow} \sum_{i=1}^{L(m)} \int_{Q} \varphi_{m}\left(m\left(x-x_{i}^{m}\right)\right) \cdot w_{i}^{m}(y) \mathrm{d} y=0 \quad \text { in } \mathscr{M}\left(\Omega ; \mathbb{R}^{N}\right)
$$

as $j \rightarrow \infty$. Recall that by construction, $\mathscr{A} w_{i}^{m}=0$ on $Q$, Hence, using that $\mathscr{A}$ is homogeneous we get

$$
\mathscr{A}\left[w_{i}^{m}\left(j m\left(\cdot-x_{i}^{m}\right)\right)\right]=0 \quad \text { in the sense of distributions on } Q_{i}^{m} .
$$

Thus, for some coefficients $c_{\alpha, \beta} \in \mathbb{N}$, using the short-hand notation $\psi_{m}(y):=\varphi_{m}(m y)$ yields

$$
\begin{aligned}
\mathscr{A} v_{j}^{m}= & \sum_{i=1}^{L(m)}\left(\mathscr{A}\left[w_{i}^{m}\left(j m\left(\cdot-x_{i}^{m}\right)\right)\right] \psi_{m}\left(\cdot-x_{i}^{m}\right)\right. \\
& \left.+\sum_{\substack{|\alpha|=k, 1 \leq|\beta| \leq k}} c_{\alpha \beta} A_{\alpha} \partial^{\alpha-\beta}\left[w_{i}^{m}\left(j m\left(\cdot-x_{i}^{m}\right)\right)\right] \partial^{\beta}\left[\psi_{m}\left(\cdot-x_{i}^{m}\right)\right]\right) \\
= & \sum_{\substack{|\alpha|=k, 1 \leq|\beta| \leq k}}\left(\sum_{i=1}^{L(m)} c_{\alpha \beta} \partial^{\alpha-\beta}\left[w_{i}^{m}\left(j m\left(\cdot-x_{i}^{m}\right)\right)\right] \partial^{\beta}\left[\psi_{m}\left(\cdot-x_{i}^{m}\right)\right]\right)
\end{aligned}
$$

in the sense of distributions on $\Omega$. Applying Lemma 2.7 to the sequence $\left(w_{i}^{m}(j m(\cdot-\right.$ $\left.\left.\left.x_{i}^{m}\right)\right)\right)_{j}$ on each cube $Q_{i}^{m}$ we get

$$
\begin{aligned}
\sum_{i=1}^{L(m)} \mathbb{1}_{Q_{i}^{m}} \cdot w_{i}^{m}\left(j m\left(\cdot-x_{i}^{m}\right)\right) & \rightarrow \sum_{i=1}^{L(m)} \mathbb{1}_{Q_{i}^{m}} \cdot f_{Q_{i}^{m}} w_{i}^{m}\left(m\left(y-x_{i}^{m}\right)\right) \mathrm{d} y \\
& =\sum_{i=1}^{L(m)} \mathbb{1}_{Q_{i}^{m}} \cdot \int_{Q} w_{i}^{m}(y) \mathrm{d} y \\
& =0 .
\end{aligned}
$$


Hence, (5.15) and the compact embedding $\mathrm{L}^{1}\left(\Omega ; \mathbb{R}^{N}\right) \stackrel{c}{\hookrightarrow} \mathrm{W}^{-1, q}\left(\Omega ; \mathbb{R}^{N}\right)$ yield

$$
\mathscr{A} v_{j}^{m}=\sum_{\substack{|\alpha|=k, 1 \leq|\beta| \leq k}}\left(\sum_{i=1}^{L(m)} c_{\alpha \beta} \partial^{\alpha-\beta}\left[w_{i}^{m}\left(j m\left(\cdot-x_{i}^{m}\right)\right)\right] \partial^{\beta}\left[\psi_{m}\left(\cdot-x_{i}^{m}\right)\right]\right) \rightarrow 0
$$

strongly in $\mathrm{W}^{-k, q}\left(\Omega ; \mathbb{R}^{N}\right)$, as $j \rightarrow \infty$.

For later use we record:

Remark 5.1. By construction, for every $m, j \in \mathbb{N}$, the function $v_{j}^{m}$ is compactly supported in $\Omega$. Up to re-scaling, we may thus assume without loss of generality that $\Omega \subset Q$ and subsequently make use of Lemma 2.15 on the $j$-indexed sequence $\left(\tilde{v}_{j}^{m}\right)$ with $m$ fixed, where $\tilde{v}_{j}^{m}$ is the zero extension of $v_{j}^{m}$ to $Q$, to find another sequence $\left(V_{j}^{m}\right) \subset \mathrm{L}^{1}\left(\Omega ; \mathbb{R}^{N}\right) \cap \operatorname{ker} \mathscr{A}$ generating the same Young measure $v^{m}($ as $j \rightarrow \infty)$.

In the next calculation we use the Lipschitz continuity of $Q_{\mathscr{A}} f(x, \cdot)$ in the second variable, equation (5.12) and the fact that the sequence $\left(v_{j}^{m}\right)$ generates the Young measure $v^{m}$ as $j \rightarrow \infty$, to get

$$
\begin{aligned}
\lim _{j \rightarrow \infty} \mathscr{G}\left[u+v_{j}^{m}\right] & =\lim _{j \rightarrow \infty} \mathscr{G}\left[z^{m}+v_{j}^{m}\right]+\mathrm{o}_{m}(1) \\
& =\sum_{i=1}^{L(m)} \int_{Q_{i}^{m}} \int_{Q} f\left(x, z_{i}^{m}+\varphi_{m}\left(m\left(x-x_{i}^{m}\right)\right) \cdot w_{i}^{m}(y)\right) \mathrm{d} y \mathrm{~d} x+\mathrm{o}_{m}(1) .
\end{aligned}
$$

By a change of variables we can estimate every double integral times $m^{d}=\mathscr{L}^{d}\left(Q_{i}^{m}\right)^{-1}$ on the last line on each cube of the mesh:

$$
\begin{array}{rl}
f_{Q_{i}^{m}} \int_{Q} & f\left(x, z_{i}^{m}+\varphi_{m}\left(m\left(x-x_{i}^{m}\right)\right) \cdot w_{i}^{m}(y)\right) \mathrm{d} y \mathrm{~d} x \\
\quad & \int_{Q} \int_{Q} f\left(x_{i}^{m}+m^{-1} x, z_{i}^{m}+\varphi_{m}(x) \cdot w_{i}^{m}(y)\right) \mathrm{d} y \mathrm{~d} x \\
& \leq \int_{Q} \int_{Q} f\left(x_{i}^{m}+m^{-1} x, z_{i}^{m}+w_{i}^{m}(y)\right) \mathrm{d} y \mathrm{~d} x+L\left\|1-\varphi_{m}\right\|_{\mathrm{L}^{1}(Q)}\left\|w_{i}^{m}\right\|_{\mathrm{L}^{1}(Q)} \\
& \leq f_{Q_{i}^{m}} \int_{Q} f\left(x, z_{i}^{m}+w_{i}^{m}(y)\right) \mathrm{d} y \mathrm{~d} x+L\left\|1-\varphi_{m}\right\|_{\mathrm{L}^{1}(Q)}\left\|w_{i}^{m}\right\|_{\mathrm{L}^{1}(Q)} \\
& :=I_{i}^{m}+I I_{i}^{m}
\end{array}
$$

where here $L$ is the $x$-uniform Lipschitz constant of $f$ with respect to the second argument. Using the modulus of continuity of $f$ from (5.10), (5.13) (twice), and $Q_{\mathscr{A}} f \leq f$, we get

$$
\begin{aligned}
I_{i}^{m} & \leq f_{Q_{i}^{m}} \int_{Q} f\left(x_{i}^{m}, z_{i}^{m}+w_{i}^{m}(y)\right) \mathrm{d} y \mathrm{~d} x+\omega\left(m^{-1}\right)\left(1+\int_{Q} f\left(x_{i}^{m}, z_{i}^{m}+w_{i}^{m}(y)\right) \mathrm{d} y\right) \\
& \leq Q_{\mathscr{A}} f\left(x_{i}^{m}, z_{i}^{m}\right)+\omega\left(m^{-1}\right)\left(1+f\left(x_{i}^{m}, z_{i}^{m}\right)\right)+\mathrm{o}_{m}(1) .
\end{aligned}
$$

Additionally, by (5.14)

$$
\sum_{i=1}^{L(m)} \mathscr{L}^{d}\left(Q_{i}^{m}\right) I I_{i}^{m}=\mathbf{o}_{m}(1)
$$


Returning to (5.16), we can employ (5.11), (5.17), (5.18) and (5.19) to further estimate

$$
\begin{aligned}
\lim _{j \rightarrow \infty} & \mathscr{G}\left[u+v_{j}^{m}\right] \\
& \leq \sum_{i=1}^{L(m)}\left\{\int_{Q_{i}^{m}} Q_{\mathscr{A}} f\left(x_{i}^{m}, z_{i}^{m}\right) \mathrm{d} x+\omega\left(m^{-1}\right)\left(\int_{Q_{i}^{m}} 1+f\left(x_{i}^{m}, z_{i}^{m}\right) \mathrm{d} x\right)\right\}+\mathrm{o}_{m}(1) \\
& \leq \sum_{i=1}^{L(m)}\left\{\int_{Q_{i}^{m}} Q_{\mathscr{A}} f\left(x_{i}^{m}, z_{i}^{m}\right) \mathrm{d} x+C \omega\left(m^{-1}\right)\left(\int_{Q_{i}^{m}} 1+\left|z_{i}^{m}\right| \mathrm{d} x\right)\right\}+\mathrm{o}_{m}(1) \\
& \leq \sum_{i=1}^{L(m)}\left\{\int_{Q_{i}^{m}} Q_{\mathscr{A}} f\left(x, z_{i}^{m}\right) \mathrm{d} x+C \omega\left(m^{-1}\right)\left(\int_{Q_{i}^{m}} 1+\left|z_{i}^{m}\right| \mathrm{d} x\right)\right\}+\mathrm{o}_{m}(1) \\
& \leq \int_{\Omega} Q_{\mathscr{A}} f\left(x, z^{m}\right) \mathrm{d} x+C \omega\left(m^{-1}\right)\left(\left\|1+\left|z^{m}\right|\right\|_{L^{1}(\Omega)}\right)+\mathrm{o}_{m}(1) \\
& =\int_{\Omega} Q_{\mathscr{A}} f(x, u(x)) \mathrm{d} x+\mathrm{o}_{m}(1),
\end{aligned}
$$

where $C>0$ and $\mathrm{o}_{m}(1)$ may change from line to line. Here, we have used the (inherited) Lipschitz continuity of $Q_{\mathscr{A}} f(x, \bullet)$ in the second variable and the fact that $\left\|u-z^{m}\right\|_{L^{1}(\Omega)}=\mathrm{o}_{m}(1)$ to pass to the last equality. Hence,

$$
\overline{\mathscr{G}}[u] \leq \inf _{m>0} \lim _{j \rightarrow \infty} \mathscr{G}\left[u+v_{j}^{m}\right] \leq \int_{\Omega} Q_{\mathscr{A}} f(x, u(x)) \mathrm{d} x .
$$

Step 3.b. The upper bound. Fix $\mu \in \mathscr{M}\left(\Omega ; \mathbb{R}^{N}\right) \cap \operatorname{ker} \mathscr{A}$. By Step 2 we may find a sequence $\left(u_{j}\right) \subset \mathrm{L}^{1}\left(\Omega ; \mathbb{R}^{N}\right)$ that area-strictly converges to $\mu \in \mathscr{M}\left(\Omega ; \mathbb{R}^{N}\right)$ with $\mathscr{A} u_{j} \rightarrow 0$ in $\mathrm{W}^{-k, q}$. Hence, by (5.20), Remark 2.6 and Corollary 2.10,

$$
\begin{aligned}
\overline{\mathscr{G}}[\mu] & \leq \liminf _{j \rightarrow \infty} \overline{\mathscr{G}}\left[u_{j}\right] \\
& \leq \limsup _{j \rightarrow \infty}\left\langle Q_{\mathscr{A}} f(x, \cdot), \delta\left[u_{j} \mathscr{L}^{d}\right]\right\rangle \\
& \leq \int_{\Omega}\left\langle Q_{\mathscr{A}} f(x, \cdot), \delta[\mu]_{x}\right\rangle \mathrm{d} x+\int_{\Omega}\left\langle\left(Q_{\mathscr{A}} f\right)^{\#}(x, \cdot), \delta[\mu]_{x}^{\infty}\right\rangle \mathrm{d} \lambda_{\delta[\mu]}(x) \\
& =\int_{\Omega} Q_{\mathscr{A}} f\left(x, \frac{\mathrm{d} \mu}{\mathrm{d} \mathscr{L}^{d}}(x)\right) \mathrm{d} x+\int_{\Omega}\left(Q_{\mathscr{A}} f\right)^{\#}\left(x, \frac{\mathrm{d} \mu^{s}}{\mathrm{~d}\left|\mu^{s}\right|}(x)\right) \mathrm{d}\left|\mu^{s}\right|(x) \\
& =\mathscr{G}_{*}[\mu] .
\end{aligned}
$$

Step 4. General continuity condition. It remains to show the upper bound in the case where we only have (1.4) instead of (5.10). As in the previous step, it suffices to show the upper bound on absolutely continuous fields. We let, for fixed $\varepsilon>0$,

$$
f^{\mathcal{\varepsilon}}(x, A):=f(x, A)+\varepsilon|A|,
$$

which is an integrand satisfying (5.10). Denote the corresponding functionals with $f^{\varepsilon}$ in place of $f$ by $\mathscr{G}^{\varepsilon}, \mathscr{G}_{*}^{\varepsilon}, \overline{\mathscr{G}^{\varepsilon}}$. Then, by the argument in Steps $1-3$,

$$
\mathscr{G}_{*}^{\varepsilon}=\overline{\mathscr{G}^{\varepsilon}} \text {. }
$$

We claim that

$$
Q_{\mathscr{A}^{k}} f^{\varepsilon} \downarrow Q_{\mathscr{A}^{k}} f \quad \text { pointwise in } \Omega \times \mathbb{R}^{N} .
$$

To see this first notice that $\varepsilon \mapsto Q_{\mathscr{A}^{k}} f^{\varepsilon}(x, A)$ is monotone decreasing for all $x \in \Omega$, $A \in \mathbb{R}^{N}$, and

$$
Q_{\mathscr{A}^{k}} f+\varepsilon|\cdot| \leq Q_{\mathscr{A}^{k}} f^{\mathcal{E}} \leq f+\varepsilon|\cdot|,
$$


which is a simple consequence of Jensen's classical inequality for $|\cdot|$. It follows that the limit

$$
g(x, A):=\inf _{\varepsilon>0} Q_{\mathscr{A}^{k}} f^{\mathcal{E}}(x, A)=\lim _{\mathcal{E} \downarrow 0} Q_{\mathscr{A}^{k}} f^{\mathcal{E}}(x, A)
$$

defines an upper semicontinuous function $g: \Omega \times \mathbb{R}^{N} \rightarrow \mathbb{R}$ with bounds

$$
Q_{\mathscr{A}^{k}} f \leq g \leq f .
$$

Furthermore, by the monotone convergence theorem, it is easy to check that $g$ is $\mathscr{A}^{k}$-quasiconvex, whereby $g=Q_{\mathscr{A}^{k}} f$ (see Corollary 2.18).

Let us now return to the proof of the upper bound on absolutely continuous fields. By construction,

$$
\overline{\mathscr{G}} \leq \overline{\mathscr{G}^{\varepsilon}}=\mathscr{G}_{*}^{\varepsilon} .
$$

The monotone convergence theorem and (5.21) yield

$$
\overline{\mathscr{G}}[u] \leq \mathscr{G}_{*}\left[u \mathscr{L}^{d}\right] \quad \text { for all } u \in \mathrm{L}^{1}\left(\Omega ; \mathbb{R}^{N}\right) \cap \operatorname{ker} \mathscr{A},
$$

after letting $\varepsilon \downarrow 0$ in (5.22).

The general upper bound then follows in a similar way to the proof under the assumption (5.10). This finishes the proof.

5.2. Proof of Theorem 1.8. The proof works the same as the proof of Theorem 1.7 with the following additional comments:

Step 1. The lower bound. Since restricting to $\mathscr{A}$-free sequences is a particular case of the more general convergence $\mathscr{A} u_{n} \rightarrow 0$ in the space $\mathrm{W}^{-k, q}\left(\Omega ; \mathbb{R}^{N}\right)$, we can still apply Step 2 in the proof of Theorem 1.7 to prove that $\mathscr{G}_{*} \leq \overline{\mathscr{G}}$, where for $\mu \in$ $\mathscr{M}\left(\Omega ; \mathbb{R}^{N}\right) \cap \operatorname{ker} \mathscr{A}$,

$$
\mathscr{G}_{*}[\mu]:=\int_{\Omega} Q_{\mathscr{A}} f\left(x, \frac{\mathrm{d} \mu}{\mathrm{d} \mathscr{L}^{d}}(x)\right) \mathrm{d} x+\int_{\Omega}\left(Q_{\mathscr{A}} f\right)^{\#}\left(x, \frac{\mathrm{d} \mu^{s}}{\mathrm{~d}\left|\mu^{s}\right|}(x)\right) \mathrm{d}\left|\mu^{s}\right|(x) .
$$

Step 2. An $\mathscr{A}$-free strictly convergent recovery sequence. In this case, this forms part of the assumptions.

Step 3.a. Upper bound on absolutely continuous $\mathscr{A}$-free fields. An immediate consequence of Remark 5.1 is that one may assume, without loss of generality, that the recovery sequence for the upper bound lies in ker $\mathscr{A}$. Thus, the upper bound on absolutely continuous fields in the constrained setting also holds.

Step 3.b. The upper bound (assuming (5.10)). The proof is the same as in the proof of Theorem 1.7.

Step 4. General continuity condition. Since assumption (5.10) is a structural property (coercivity) of the integrand and the arguments do not depend on the underlying space of measures, the argument remains the same as in the proof of Theorem 1.7 .

\section{APPENDiX A. PROOFS OF THE LOCALIZATION PRINCIPLES}

In this appendix we prove Proposition 2.24 and Proposition 2.25.

Proof of Proposition 2.24: In the following we adapt the main steps in proof of the localization principle at regular points which is contained in Proposition 1 of [Rin12]. The statement on the existence of an $\mathscr{A}$-free and periodic generating sequence is proved in detail.

Let $\mu_{j} \in \mathscr{M}\left(\Omega ; \mathbb{R}^{N}\right)$ be the sequence of asymptotically $\mathscr{A}$-free measures which generates $v$. In the following steps, for an open $\Omega^{\prime} \subset \mathbb{R}^{d}$, we will often identify a 
measure $\mu \in \mathscr{M}\left(\Omega^{\prime} ; \mathbb{R}^{N}\right)$ with its zero extension in $\mathscr{M}_{\mathrm{loc}}\left(\mathbb{R}^{d} ; \mathbb{R}^{N}\right)$, and similarly for a Young measure $\sigma \in \mathbf{Y}\left(\Omega^{\prime} ; \mathbb{R}^{N}\right)$ and its zero extension in $\mathbf{Y}_{\text {loc }}\left(\mathbb{R}^{d} ; \mathbb{R}^{N}\right)$.

Step 1. We start by showing that, for every $r>0$, there exists a subsequence of $j$ 's (the choice of subsequence might depend on $r$ ) such that

$$
r^{-d} T_{\#}^{\left(x_{0}, r\right)} \mu_{j} \stackrel{\mathbf{Y}}{\rightarrow} \sigma^{(r)} \quad \text { in } \mathbf{Y}_{\mathrm{loc}}\left(\mathbb{R}^{d} ; \mathbb{R}^{N}\right)
$$

Moreover, for $\mathscr{L}^{d}$-a.e. $x_{0} \in \Omega$, one can show that a uniform bound

$$
\sup _{r>0}\left\langle\left\langle\mathbb{1}_{K} \otimes|\cdot|, \sigma^{(r)}\right\rangle<\infty \quad \text { for every } K \Subset \mathbb{R}^{d}\right.
$$

holds; thus, by Lemma 2.5, there exists a sequence of positive numbers $r_{m} \downarrow 0$ and a Young measure $\sigma$ for which

$$
\sigma^{\left(r_{m}\right)} \stackrel{*}{\rightarrow} \sigma \quad \text { in } \mathbf{Y}_{\mathrm{loc}}\left(\mathbb{R}^{d} ; \mathbb{R}^{N}\right) .
$$

Step 2. For an arbitrary measure $\gamma \in \mathscr{M}\left(\Omega ; \mathbb{R}^{N}\right)$, the Radon-Nykodým differentiation theorem yields

$$
r^{-d} T_{\#}^{\left(x_{0}, r\right)} \gamma=\frac{\mathrm{d} \gamma}{\mathrm{d} \mathscr{L}^{d}}\left(x_{0}+r \cdot\right) \mathscr{L}^{d}+\frac{\mathrm{d} \gamma}{\mathrm{d}\left|\gamma^{s}\right|}\left(x_{0}+r \cdot\right) r^{-d} T_{\#}^{\left(x_{0}, r\right)}\left|\gamma^{s}\right| .
$$

Consider $\sigma^{(r)}$ as an element of $\mathbf{Y}\left(Q ; \mathbb{R}^{N}\right)$. Fix $\varphi \otimes h \in \mathrm{C}(\bar{Q}) \times \operatorname{Lip}\left(\mathbb{R}^{N}\right)$. Using a simple change of variables, we get

$$
\begin{aligned}
\left\langle\varphi \otimes h, \sigma^{(r)}\right\rangle= & \lim _{j \rightarrow \infty}\left(\int_{Q} \varphi(y) \cdot h\left(\frac{\mathrm{d} \mu_{j}}{\mathrm{~d} \mathscr{L}^{d}}\left(x_{0}+r y\right)\right) \mathrm{d} y\right. \\
& \left.\quad+\int_{\bar{Q}} \varphi(y) \cdot h^{\infty}\left(\frac{\mathrm{d} \mu_{j}}{\mathrm{~d}\left|\mu_{j}^{s}\right|}\left(x_{0}+r y\right)\right) \mathrm{d}\left(r^{-d} T_{\#}^{\left(x_{0}, r\right)}\left|\mu_{j}^{s}\right|\right)(y)\right) \\
= & r^{-d} \lim _{j \rightarrow \infty}\left(\int_{Q_{r}\left(x_{0}\right)} \varphi \circ T^{\left(x_{0}, r\right)}(x) \cdot h\left(\frac{\mathrm{d} \mu_{j}}{\mathrm{~d} \mathscr{L}^{d}}(x)\right) \mathrm{d} x\right. \\
& \left.\quad+\int_{\overline{Q_{r}\left(x_{0}\right)}} \varphi \circ T^{\left(x_{0}, r\right)}(x) \cdot h^{\infty}\left(\frac{\mathrm{d} \mu_{j}}{\mathrm{~d}\left|\mu_{j}^{s}\right|} x\right) \mathrm{d}\left|\mu_{j}^{s}\right|(x)\right) \\
= & \left.r^{-d} \|\left(\varphi \circ T^{\left(x_{0}, r\right)}\right) \otimes h, v\right\rangle .
\end{aligned}
$$

Step 3. We now let $r=r_{m}$ in (A.3) and quantify its values as $m \rightarrow \infty$. This will allow us to characterize $\sigma$ in terms of $v$.

Let $\left\{g_{l}:=\varphi_{l} \otimes h_{l}\right\}_{l} \subset \mathrm{C}(\bar{Q}) \times \operatorname{Lip}\left(\mathbb{R}^{N}\right)$ be the dense subset of $\mathbf{E}\left(Q ; \mathbb{R}^{N}\right)$ provided by Lemma 2.4 and further assume that $x_{0}$ verifies the following properties: $x_{0}$ is a Lebesgue point of the functions

$$
x \mapsto\left\langle h_{l}, v_{x}\right\rangle+\left\langle h_{l}^{\infty}, v_{x}^{\infty}\right\rangle \frac{\mathrm{d} \lambda_{v}}{\mathrm{~d} \mathscr{L}^{d}}(x), \quad \text { for all } l \in \mathbb{N},
$$

and $x_{0}$ is a regular point of the measure $\lambda_{v}$, that is,

$$
\frac{\mathrm{d} \lambda_{v}^{s}}{\mathrm{~d} \mathscr{L}^{d}}\left(x_{0}\right)=\lim _{r \downarrow 0} \frac{\lambda_{v}^{s}\left(Q_{r}\left(x_{0}\right)\right)}{r^{d}}=0 .
$$


Consider $\sigma$ as an element of $\mathbf{Y}\left(Q ; \mathbb{R}^{N}\right)$. Setting $r=r_{m}$ in (A.3) and letting $m \rightarrow \infty$ we get

$$
\begin{aligned}
\left\langle g_{l}, \sigma\right\rangle= & \lim _{m \rightarrow \infty} r_{m}^{-d}\left\langle\varphi_{l} \circ T^{\left(x_{0}, r_{m}\right)} \otimes h_{l}, v\right\rangle \\
= & \lim _{m \rightarrow \infty}\left(f_{Q_{r_{m}}\left(x_{0}\right)} \varphi_{l}\left(\frac{x-x_{0}}{r_{m}}\right)\left[\left\langle h_{l}, v_{x}\right\rangle+\left\langle h_{l}^{\infty}, v_{x}^{\infty}\right\rangle \frac{\mathrm{d} \lambda_{v}}{\mathrm{~d} \mathscr{L}^{d}}(x)\right] \mathrm{d} x\right. \\
& \left.\quad+\frac{1}{r^{d}} \int_{\overline{Q_{r_{m}}\left(x_{0}\right)}} \varphi_{l}\left(\frac{x-x_{0}}{r_{m}}\right)\left\langle h_{l}^{\infty}, v_{x}^{\infty}\right\rangle \mathrm{d} \lambda_{v}^{s}(x)\right) \\
= & \int_{Q}\left\langle g_{l}(y, \cdot), v_{x_{0}}\right\rangle \mathrm{d} y+\int_{Q}\left\langle g_{l}^{\infty}(y, \cdot), v_{x_{0}}^{\infty}\right\rangle \frac{\mathrm{d} \lambda_{v}}{\mathrm{~d} \mathscr{L}^{d}}\left(x_{0}\right) \mathrm{d} y .
\end{aligned}
$$

Here, we have used (A.4) and the Dominated Convergence Theorem to pass to the limit in the first summand, and with the help of (A.5), we used that

$$
\int_{\overline{Q_{r}\left(x_{0}\right)}} \varphi_{l}\left(\frac{x-x_{0}}{r}\right)\left\langle h_{l}^{\infty}, v_{x}^{\infty}\right\rangle \mathrm{d} \lambda_{v}^{s}(x) \leq\|\varphi\|_{\infty} \cdot \operatorname{Lip}\left(h_{l}\right) \cdot \lambda_{v}^{s}\left(Q_{r}\left(x_{0}\right)\right)=\mathrm{o}\left(r^{d}\right)
$$

to neglect the second summand in the limiting process.

Since the set $\left\{g_{l}\right\}$ separates $\mathbf{Y}\left(Q ; \mathbb{R}^{N}\right)$, Lemma 2.4 tells us that $\sigma_{y}=v_{x_{0}}, \sigma_{y}^{\infty}=$ $v_{x_{0}}^{\infty}, \lambda_{\sigma}=\frac{\mathrm{d} \lambda_{v}}{\mathrm{~d} \mathscr{L}^{d}}\left(x_{0}\right) \mathscr{L}^{d}$ for $\mathscr{L}^{d}$ a-e. $y \in Q$, and that $\lambda_{\sigma}^{s}$ is the zero measure in $\mathscr{M}(\bar{Q})$, as desired.

Step 4. We use a diagonalization principle (where $j$ is the fast index with respect to $m$ ) to find a subsequence $\left(\mu_{j(m)}\right)$ such that

$$
\gamma_{m}:=r_{m}^{-d} T_{\#}^{\left(x_{0}, r_{m}\right)} \mu_{j(m)} \stackrel{\mathbf{Y}}{\rightarrow} \sigma \quad \text { in } \mathbf{Y}_{\mathrm{loc}}\left(\mathbb{R}^{d} ; \mathbb{R}^{N}\right) .
$$

Step 5. Up to this point, the localization principle presented in Proposition 1 of [Rin12] has been adapted to Young measures without imposing any differential constraint. Here we additionally require $\sigma$ to be an $\mathscr{A}^{k}$-free Young measure; this is achieved by showing that $\left(\gamma_{m}\right)$ is asymptotically $\mathscr{A}^{k}$-free (on bounded subsets of $\mathbb{R}^{d}$ ). To this end let us note that

$$
\mathscr{A} \mu_{j}=\theta_{j}
$$

with $\left\|\theta_{j}\right\|_{W^{-k, q}} \rightarrow 0$ as $j \rightarrow \infty$. By scaling we can write

$$
\mathscr{A}^{k} \gamma_{m}=r_{m}^{-d} \mathscr{A}^{k}\left(T_{\#}^{x_{0}, r_{m}} \mu_{j(m)}\right)=-\sum_{h=0}^{k-1} \mathscr{A}^{h}\left(r^{k-h} r_{m}^{-d} T_{\#}^{\left(x_{0}, r\right)} \mu_{j(m)}\right)+r_{m}^{k-d} T_{\#}^{\left(x_{0}, r_{m}\right)} \theta_{j(m)} .
$$

Since $\left\|r_{m}^{k-d} T_{\#}^{\left(x_{0}, r_{m}\right)} \theta_{j}\right\|_{W^{-k, q}} \leq C(m)\left\|\theta_{j}\right\|_{W^{-k, q}}$ and for every open $U \Subset \mathbb{R}^{d}$ there exists a positive constant $C_{U}$ such that

$$
\sup _{m \in \mathbb{N}} r_{m}^{-d} T_{\#}^{\left(x_{0}, r_{m}\right)}\left|\mu_{j}\right|(U) \leq C_{U},
$$

arguing as in Proposition 2.16 we can choose a further subsequence $j(m) \rightarrow \infty$ such that

$$
\mathscr{A}^{k} \gamma_{m}=\mathscr{A}^{k}\left(r_{m}^{-d} T_{\#}^{\left(x_{0}, r\right)} \mu_{j(m)}\right) \rightarrow 0 \quad \text { in } \mathrm{W}_{\mathrm{loc}}^{-k, q}\left(\mathbb{R}^{d}\right)
$$

and this shows that $\sigma$ is an $\mathscr{A}^{k}$-free Young measure.

Step 6. So far we have shown that $[\sigma]=A_{0} \mathscr{L}^{d}$ with

$$
A_{0}:=\left\langle\mathrm{id}, v_{x_{0}}\right\rangle+\left\langle\mathrm{id}, v_{x_{0}}^{\infty}\right\rangle \frac{\mathrm{d} \lambda_{v}}{\mathrm{~d} \mathscr{L}^{d}}\left(x_{0}\right) \quad \in \mathbb{R}^{N},
$$


and that $\sigma$ is generated by a sequence $\left(\mu_{j}\right) \subset \mathscr{M}\left(Q ; \mathbb{R}^{N}\right)$ satisfying $\mathscr{A}^{k} \mu_{j} \rightarrow 0$. Note that without loss of generality we may assume that the $\mu_{j}$ 's are of the form $u_{j} \mathscr{L}^{d}$ where $u_{j} \in \mathrm{L}^{1}\left(Q ; \mathbb{R}^{N}\right)$. Indeed, since

$$
\begin{gathered}
\gamma_{R}:=T_{\#}^{\left(x_{0}, R\right)} \mu_{j} \rightarrow \mu_{j} \quad \text { area strictly in } \mathscr{M}_{\mathrm{loc}}\left(\mathbb{R}^{d} ; \mathbb{R}^{N}\right), \\
\left\|\mathscr{A}^{k}\left(\gamma_{R}-\mu_{j}\right)\right\|_{\mathrm{W}_{\mathrm{loc}}^{-k, q}\left(\mathbb{R}^{d}\right)} \rightarrow 0 \quad \text { as } R \uparrow 1,
\end{gathered}
$$

and

$$
\begin{gathered}
\gamma_{R} * \rho_{\varepsilon} \rightarrow \gamma_{R} \quad \text { area strictly in } \mathscr{M}_{\mathrm{loc}}\left(\mathbb{R}^{d} ; \mathbb{R}^{N}\right), \\
\left\|\mathscr{A}^{k}\left(\gamma_{R}-\gamma_{R} * \rho_{\varepsilon}\right)\right\|_{\mathrm{W}_{\mathrm{loc}}^{-k, q}\left(\mathbb{R}^{d}\right)} \rightarrow 0 \quad \text { as } \varepsilon \downarrow 0,
\end{gathered}
$$

we might use a diagonalization argument (relying on the weak*-metrizability of bounded subsets of $\mathbf{E}\left(Q ; \mathbb{R}^{N}\right)^{*}$ and Remarks 2.2,2.6), where $\varepsilon$ appears as the faster index with respect to $R$, to find a sequence with elements $u_{j}:=\gamma_{R(j)} * \rho_{\varepsilon(R(j))}$ such that

$$
u_{j} \mathscr{L}^{d} \stackrel{\mathbf{Y}}{\rightarrow} \sigma \in \mathbf{Y}_{\mathrm{loc}}\left(\mathbb{R}^{d} ; \mathbb{R}^{N}\right) \quad \text { and } \quad \mathscr{A}^{k} u_{j} \rightarrow 0 \quad \text { in } \mathrm{W}_{\mathrm{loc}}^{-k, q}\left(\mathbb{R}^{d}\right)
$$

Using (2.3), we get

$$
\left|u_{j}\right| \mathscr{L}^{d} \stackrel{*}{\rightarrow}|[\sigma]|=\left|A_{0}\right| \mathscr{L}^{d} \quad \text { in } \mathscr{M}_{\mathrm{loc}}\left(\mathbb{R}^{d}\right) .
$$

Hence, $\left|u_{j}\right| \mathscr{L}^{d} \stackrel{*}{\rightarrow} \Lambda$ in $\mathscr{M}(\bar{Q})$ with $\Lambda(\partial Q)=0$. We are now in position to apply Lemma 2.15 to the sequences $\left(u_{j}\right)$ and $\left(v_{j}:=A_{0}\right)$ to find a sequence $z_{j} \in \mathrm{C}_{\mathrm{per}}^{\infty}\left(Q ; \mathbb{R}^{N}\right) \cap$ $\operatorname{ker} \mathscr{A}^{k}$ with $\int_{Q} z_{j} \mathrm{~d} y=0$ and such that (up to taking a subsequence)

$$
\left(A_{0}+z_{j}\right) \mathscr{L}^{d} \stackrel{\mathbf{Y}}{\rightarrow} \sigma \quad \text { in } \mathbf{Y}\left(Q ; \mathbb{R}^{N}\right)
$$

Since the properties of $x_{0}$ that were involved in Steps 1-3 are valid at $\mathscr{L}^{d}$-a.e. $x_{0} \in \Omega$, the sought localization principle at regular points is proved.

Proof of Proposition 2.25: The proof of the localization principle at singular points resembles the one for regular points, with a few exceptions:

Step 1. In comparison to Step 1 from the regular localization principle, we here chose $c_{r}\left(x_{0}\right):=\left|\lambda_{v}^{s}\right|\left(Q_{r}\left(x_{0}\right)\right)^{-1}>0$ and we define $\sigma^{r}$ as

$$
c_{r}\left(x_{0}\right) T_{\#}^{\left(x_{0}, r\right)} \mu_{j} \stackrel{\mathbf{Y}}{\rightarrow} \sigma^{(r)} \quad \text { in } \mathbf{Y}_{\mathrm{loc}}\left(\mathbb{R}^{d} ; \mathbb{R}^{N}\right) .
$$

Moreover, by [Pre87, Lemma 2.4 and Theorem 2.5] and (A.12) below, at $\lambda_{v}^{s}$-a.e. $x_{0} \in \Omega$, it is possible to show that

$$
\sup _{r>0}\left\langle\left\langle\mathbb{1}_{K} \otimes|\cdot|, \sigma^{(r)}\right\rangle=\sup _{r>0} \frac{\left|\lambda_{v}^{s}\right|\left(x_{0}+r K\right)+\mathscr{L}^{d}\left(x_{0}+r K\right)}{\left|\lambda_{v}^{s}\right|\left(Q_{r}\left(x_{0}\right)\right)}<\infty \quad \text { for every } K \Subset \mathbb{R}^{d} .\right.
$$

By compactness of $\mathbf{Y}_{\mathrm{loc}}\left(\mathbb{R}^{d} ; \mathbb{R}^{N}\right)$, see Lemma 2.5 , there exists a sequence of positive numbers $r_{m} \downarrow 0$ and a Young measure $\sigma$ for which

$$
\sigma^{\left(r_{m}\right)} \stackrel{*}{\rightarrow} \sigma \quad \text { in } \mathbf{Y}_{\mathrm{loc}}\left(\mathbb{R}^{d} ; \mathbb{R}^{N}\right)
$$


Step 2. The calculations of the second step, for the constant $c_{r}\left(x_{0}\right)$, is

$$
\begin{aligned}
\left\langle\varphi \otimes h, \sigma^{(r)}\right\rangle= & \lim _{j \rightarrow \infty}\left(\int_{Q} \varphi(y) \cdot h\left(c_{r}\left(x_{0}\right) r^{d} \frac{\mathrm{d} \mu_{j}}{\mathrm{~d} \mathscr{L}^{d}}\left(x_{0}+r y\right)\right) \mathrm{d} y\right. \\
& \left.\quad+\int_{\bar{Q}} \varphi(y) \cdot h^{\infty}\left(c_{r}\left(x_{0}\right) r^{d} \frac{\mathrm{d} \mu_{j}}{\mathrm{~d}\left|\mu_{j}^{s}\right|}\left(x_{0}+r y\right)\right) \mathrm{d}\left(r^{-d} T_{\#}^{\left(x_{0}, r\right)}\left|\mu_{j}^{s}\right|\right)(y)\right) \\
= & r^{-d} \lim _{j \rightarrow \infty}\left(\int_{Q_{r}\left(x_{0}\right)} \varphi \circ T^{\left(x_{0}, r\right)}(x) \cdot h\left(c_{r}\left(x_{0}\right) r^{d} \frac{\mathrm{d} \mu_{j}}{\mathrm{~d} \mathscr{L}^{d}}(x)\right) \mathrm{d} x\right. \\
& \left.\quad+\int_{Q_{r}\left(x_{0}\right)} \varphi \circ T^{\left(x_{0}, r\right)}(x) \cdot h^{\infty}\left(c_{r}\left(x_{0}\right) r^{d} \frac{\mathrm{d} \mu_{j}}{\mathrm{~d}\left|\mu_{j}^{s}\right|}(x)\right) \mathrm{d}\left|\mu_{j}^{s}\right|(x)\right) \\
= & r^{-d}\left\langle\varphi \circ T^{\left(x_{0}, r\right)} \otimes h\left(c_{r}\left(x_{0}\right) r^{d} \cdot\right), v\right\rangle .
\end{aligned}
$$

Step 3. The assumptions of the third step are substituted by assuming that $x_{0}$ is a $\lambda_{v}^{s}$-Lebesgue point of the functions

$$
x \mapsto\left\langle|\cdot|, v_{x}^{\infty}\right\rangle, \quad x \mapsto\left\langle h_{l}^{\infty}, v_{x}^{\infty}\right\rangle \quad \text { for all } l \in \mathbb{N} .
$$

We further require that

$$
\lim _{r \downarrow 0} \frac{r^{d}}{\lambda_{v}^{s}\left(Q_{r}\left(x_{0}\right)\right)}=\lim _{r \downarrow 0} c_{r}\left(x_{0}\right) r^{d}=0
$$

and that

$$
\lim _{r \downarrow 0} c_{r}\left(x_{0}\right) \int_{Q_{r}\left(x_{0}\right)}\left[\left\langle|\cdot|, v_{x}\right\rangle+\left\langle|\cdot|, v_{x}^{\infty}\right\rangle \frac{\mathrm{d} \lambda_{v}}{\mathrm{~d} \mathscr{L}}(x)\right] \mathrm{d} x=0 .
$$

Hence, defining $S:=\left\{x_{0} \in \Omega:\right.$ (A.11), (A.12) and (A.13) hold $\}$, we have $\lambda_{v}^{s}(\Omega \backslash$ $S)=0$.

Fix $x_{0} \in S$. Setting $r=r_{m}$ in (A.10) and letting $m \rightarrow \infty$ gives

$$
\begin{aligned}
\left\langle\mathbb{1}_{Q} \otimes|\cdot|, \sigma\right\rangle= & \lim _{m \rightarrow \infty}\left\langle\left\langle\mathbb{1}_{Q} \otimes|\cdot|, \sigma^{\left(r_{m}\right)}\right\rangle\right. \\
= & \lim _{m \rightarrow \infty} c_{m}\left(x_{0}\right)\left(\int_{Q_{r_{m}}\left(x_{0}\right)}\left[\left\langle|\cdot|, v_{x}\right\rangle+\left\langle|\cdot|, v_{x}^{\infty}\right\rangle \frac{\mathrm{d} \lambda_{v}}{\mathrm{~d} \mathscr{L}}(x)\right] \mathrm{d} x\right. \\
& \left.\quad+\int_{\overline{Q_{r_{m}}\left(x_{0}\right)}}\left\langle|\cdot|, v_{x}^{\infty}\right\rangle \mathrm{d} \lambda_{v}^{s}(x)\right) \\
= & \left\langle|\cdot|, v_{x_{0}}^{\infty}\right\rangle \lim _{m \rightarrow \infty}\left(\int_{\bar{Q}} \mathrm{~d}\left(c_{m}\left(x_{0}\right) T_{\#}^{\left(x_{0}, r_{m}\right)} \lambda_{v}^{s}\right)(y)\right) \\
= & \int_{\bar{Q}}\left\langle|\cdot|, v_{x_{0}}^{\infty}\right\rangle \mathrm{d} \gamma(y), \quad \text { for some } \gamma \in \operatorname{Tan}\left(\lambda_{v}^{s}, x_{0}\right),
\end{aligned}
$$

where we have used that $x_{0} \in S$. Moreover

$$
\gamma(\bar{Q})=\left\langle\left\langle\mathbb{1}_{\bar{Q}} \otimes|\cdot|, \sigma\right\rangle \geq \lim _{m \rightarrow \infty} \frac{\left|\lambda_{v}^{s}\right|\left(Q_{r_{m}}\left(x_{0}\right)\right)}{\left|\lambda_{v}^{s}\right|\left(Q_{r_{m}}\left(x_{0}\right)\right)}=1\right.
$$

which implies $\gamma \neq 0$. Testing with $g_{l}=\varphi_{l} \otimes h_{l}$, we obtain by (A.11) and a similar argument to the one above, that

$$
\left\langle g_{l}, \sigma\right\rangle=\int_{\bar{Q}} \varphi_{l}(y)\left\langle h_{l}^{\infty}, v_{x_{0}}^{\infty}\right\rangle \mathrm{d} \gamma(y) .
$$

From the above equations we deduce that that $\sigma_{y}=\delta_{0}$ for $\mathscr{L}^{d}$-a.e. $y \in Q, \sigma_{y}^{\infty}=v_{x_{0}}^{\infty}$ and $\lambda_{\sigma}=\gamma \in \operatorname{Tan}\left(\lambda_{v}^{s}, x_{0}\right) \backslash\{0\}$. 
Step 4. The arguments of Step 4 remain unchanged except that this time one gets

$$
\gamma_{m}:=c_{m} T_{\#}^{\left(x_{0}, r_{m}\right)} \mu_{j(m)} \stackrel{\mathbf{Y}}{\rightarrow} \sigma \quad \text { in } \mathbf{Y}\left(Q, \mathbb{R}^{N}\right) ;
$$

Step 5. This is similar to the corresponding step in the proof of the regular localization principle.

Step 6. Differently from the case at regular points, we want to additionally show $\lambda_{\sigma}(Q)=1$ and $\lambda_{\sigma}(\partial Q)=0$. There exists $0<\varepsilon<1$ such that $\lambda_{\sigma}\left(\partial Q_{\varepsilon}\right)=0$. Up to taking $r^{\prime}=\varepsilon r$ (and thus as $r_{m}^{\prime}=r_{m} \varepsilon$ ) in the arguments of Steps 1-4 above we may assume without loss of generality that $\lambda_{\sigma}(\partial Q)=0$ and $\lambda_{\sigma}(Q)=1$. This proves the localization principle at singular points.

\section{REFERENCES}

[AB97] J. J. Alibert and G. Bouchitté, Non-uniform integrability and generalized Young measures, J. Convex Anal. 4 (1997), 129-147.

[AD92] L. Ambrosio and G. Dal Maso, On the relaxation in $\mathrm{BV}\left(\Omega ; \mathbf{R}^{m}\right)$ of quasi-convex integrals, J. Funct. Anal. 109 (1992), 76-97.

[AFP00] L. Ambrosio, N. Fusco, and D. Pallara, Functions of Bounded Variation and FreeDiscontinuity Problems, Oxford Mathematical Monographs, Oxford University Press, 2000.

[AR16] A. Arroyo-Rabasa, Relaxation and optimization for linear-growth convex integral functionals under PDE constraints, J. Funct. Anal. 273 (2017), 2388-2427.

[BCMS13] M. Baía, M. Chermisi, J. Matias, and P. M. Santos, Lower semicontinuity and relaxation of signed functionals with linear growth in the context of $\mathscr{A}$-quasiconvexity, Calc. Var. Partial Differential Equations 47 (2013), 465-498.

[BM84] J. M. Ball and F. Murat, $W^{1, p}$-quasiconvexity and variational problems for multiple integrals, J. Funct. Anal. 58 (1984), no. 3, 225-253.

[BFT00] A. C. Barroso, I. Fonseca, and R. Toader, A relaxation theorem in the space of functions of bounded deformation, Ann. Scuola Norm. Sup. Pisa Cl. Sci. 29 (2000), 19-49.

[Bi103] M. Bildhauer, Convex variational problems, Lecture Notes in Mathematics, vol. 1818, Springer, 2003.

[Dac82] B. Dacorogna, Weak Continuity and Weak Lower Semicontinuity for Nonlinear Functionals, Lecture Notes in Mathematics, vol. 922, Springer, 1982.

[DR16a] G. De Philippis and F. Rindler, Characterization of generalized Young measures generated by symmetric gradients, Arch. Ration. Mech. Anal. 224, (2017), no. 3, 1087-1125.

[DR16b] On the structure of $\mathscr{A}$-free measures and applications, Ann. of Math. 184 (2016), 1017-1039.

[DiP85] R. J. DiPerna, Compensated compactness and general systems of conservation laws, Trans. Amer. Math. Soc. 292 (1985), 383-420.

[DM87] R. J. DiPerna and A. J. Majda, Oscillations and concentrations in weak solutions of the incompressible fluid equations, Comm. Math. Phys. 108 (1987), 667-689.

[FLM04] I. Fonseca, G. Leoni, and S. Müller, $\mathscr{A}$-quasiconvexity: weak-star convergence and the gap, Ann. Inst. H. Poincaré Anal. Non Linéaire 21 (2004), no. 2, 209-236.

[FM93] I. Fonseca and S. Müller, Relaxation of quasiconvex functionals in $\mathrm{BV}\left(\Omega, \mathbf{R}^{p}\right)$ for integrands $f(x, u, \nabla u)$, Arch. Ration. Mech. Anal. 123 (1993), 1-49.

[FM99] _ A -quasiconvexity, lower semicontinuity, and Young measures, SIAM J. Math. Anal. 30 (1999), 1355-1390.

[KK11] B. Kirchheim and J. Kristensen, Automatic convexity of rank-1 convex functions, Comptes Rendus Mathematique 349 (2011), 407-409.

[KK16] On rank-one convex functions that are homogeneous of degree one, Arch. Ration. Mech. Anal. 221 (2016), 527-558.

[KR10a] J. Kristensen and F. Rindler, Characterization of generalized gradient Young measures generated by sequences in $W^{1,1}$ and BV, Arch. Ration. Mech. Anal. 197 (2010), 539-598, Erratum: Vol. 203 (2012), 693-700.

[KR10b] , Relaxation of signed integral functionals in BV, Calc. Var. Partial Differential Equations 37 (2010), 29-62. 
[Mat95] P. Mattila, Geometry of Sets and Measures in Euclidean Spaces, Cambridge Studies in Advanced Mathematics, vol. 44, Cambridge University Press, 1995.

[Mor66] C. B. Morrey, Jr., Multiple Integrals in the Calculus of Variations, Grundlehren der mathematischen Wissenschaften, vol. 130, Springer, 1966.

[Mül87] S. Müller, Homogenization of nonconvex integral functionals and cellular elastic materials, Arch. Rational Mech. Anal. 99 (1987), no. 3, 189-212.

[Mur78] F. Murat, Compacité par compensation, Ann. Scuola Norm. Sup. Pisa Cl. Sci. 5 (1978), 489-507.

[Mur79] Compacité par compensation. II, Proceedings of the International Meeting on Recent Methods in Nonlinear Analysis (Rome, 1978), Pitagora, 1979, pp. 245-256.

[Mur81] Compacité par compensation: condition nécessaire et suffisante de continuité faible sous une hypothèse de rang constant, Ann. Scuola Norm. Sup. Pisa Cl. Sci. 8 (1981), 69-102.

[Pre87] D. Preiss, Geometry of measures in $\mathbf{R}^{n}$ : distribution, rectifiability, and densities, Ann. of Math. 125 (1987), 537-643.

[Rin11] F. Rindler, Lower semicontinuity for integral functionals in the space of functions of bounded deformation via rigidity and Young measures, Arch. Ration. Mech. Anal. 202 (2011), 63-113.

[Rin12] Lower semicontinuity and Young measures in BV without Alberti's Rank-One Theorem, Adv. Calc. Var. 5 (2012), 127-159.

[Tar79] L. Tartar, Compensated compactness and applications to partial differential equations, Nonlinear analysis and mechanics: Heriot-Watt Symposium, Vol. IV, Res. Notes in Math., vol. 39, Pitman, 1979, pp. 136-212.

[Tar83] - The compensated compactness method applied to systems of conservation laws, Systems of nonlinear partial differential equations (Oxford, 1982), NATO Adv. Sci. Inst. Ser. C Math. Phys. Sci., vol. 111, Reidel, 1983, pp. 263-285.

A.A.-R.: MAthematisches Institut, UniVersität LeIPZIG, 53115 Bonn, Germany.

E-mail address: arroyo@math.uni-leipzig.de

G.D.P: SISSA, Via BoNOMEA 265, 34136 TRIESTE, ITALY.

E-mail address: guido.dephilippis@sissa.it

F.R.: Mathematics Institute, University of Warwick, Coventry CV4 7AL, UK.

E-mail address: F.Rindler@warwick.ac.uk 